\title{
COUNTERACTING TRAFFIC CONGESTION USING INTELLIGENT DRIVER FEEDBACK
}

\author{
A Thesis presented to \\ the Faculty of the Graduate School \\ at the University of Missouri
}

In Partial Fulfillment

of the Requirements for the Degree

Masters of Science in Civil Engineering

\author{
by \\ DAVID K. DRUM
}

Dr. Timothy C. Matisziw, Thesis Supervisor

MAY 2014 
(C) Copyright by David K. Drum 2014 All Rights Reserved 
The undersigned, appointed by the Dean of the Graduate School, have examined the thesis entitled:

\section{COUNTERACTING TRAFFIC CONGESTION USING INTELLIGENT DRIVER FEEDBACK}

presented by David K. Drum

A candidate for the degree of Masters of Science in Civil Engineering and hereby certify that, in their opinion, it is worthy of acceptance.

Dr. Timothy C. Matisziw

Dr. Carlos Sun

Dr. Praveen K. Edara

Dr. Dmitry Korkin

Dr. Ron McGarvey 


\section{DEDICATION}

Dedicated to

Marta and Clara

Thanks to

David Olson

Dan Carr

Jason Cameron

Chip Byers

Jim Long 


\section{ACKNOWLEDGEMENTS}

Thanks to Tim Matisziw for his support and advice and to Dmitry Korkin for guidance regarding machine learning techniques. 


\section{TABLE OF CONTENTS}

Acknowledgements $\ldots \ldots \ldots \ldots \ldots \ldots \ldots \ldots \ldots$ ii

List of Tables $\ldots \ldots \ldots \ldots \ldots \ldots \ldots \ldots \ldots \ldots \ldots \ldots \ldots$

List of Figures $\ldots \ldots \ldots \ldots \ldots \ldots \ldots \ldots \ldots \ldots \ldots \ldots \ldots \ldots$

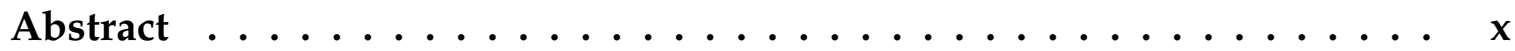

Chapter

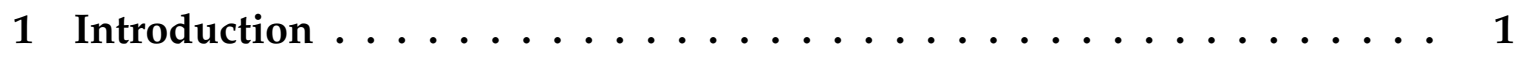

1.1 The Automobile's Unintended Consequences . . . . . . . . . . . . . 4

1.2 The Need for Autonomous Vehicles $\ldots \ldots \ldots \ldots$

1.3 The State Of The Autonomous Vehicle $\ldots \ldots \ldots$

1.4 Personal Motivations . . . . . . . . . . . . . . . . . . . . . . 11

1.5 Organization of this Thesis . . . . . . . . . . . . . 12

2 Literature Review . . . . . . . . . . . . . . . . . . 14

2.1 Congestion $\ldots \ldots \ldots \ldots \ldots \ldots \ldots \ldots \ldots \ldots \ldots \ldots$

2.1.1 Impacts of Congestion $\ldots \ldots \ldots \ldots$

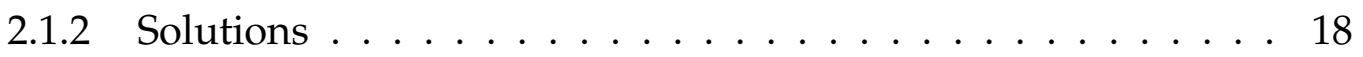

2.1.3 Congestion Shockwaves And Traffic Oscillations . . . . . . . 20

2.1.4 Mathematical Solubility . . . . . . . . . . . . . . . 22 
2.2 Driver Assistance and Feedback Systems _ . . . . . . . . . . 23

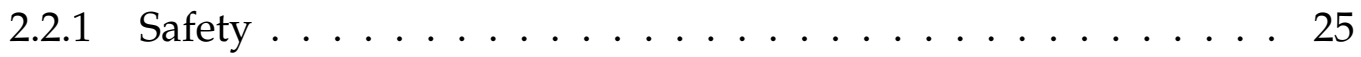

2.2.2 Adaptive Cruise Control . . . . . . . . . . . . . . . . . 26

2.2.3 Smart Phones . . . . . . . . . . . . . . . . . . 27

2.3 Creating A Feedback Heuristic $\ldots \ldots \ldots \ldots \ldots$

2.3.1 Intelligent Driver Model . . . . . . . . . . . . . . . . 33

2.3.2 Discrete Wavelet Transformations . . . . . . . . . . . . . . . 37

2.3.3 Machine Learning Algorithms . . . . . . . . . . . . . . . 38

2.3.4 Implications for the Present Work . . . . . . . . . . . . . 42

3 Methods ....................... 46

3.1 Implementation Of The Intelligent Driver Model $\ldots \ldots \ldots$

3.1.1 Simulator Architecture . . . . . . . . . . . . . . . 47

3.2 Simulation $\ldots \ldots \ldots \ldots \ldots \ldots \ldots \ldots \ldots \ldots \ldots \ldots$

3.2.1 Virtual Macroscopic Traffic Detectors . . . . . . . . . . . . . 54

3.2.2 Vehicle Merging . . . . . . . . . . . . . . . . . 55

3.2.3 Sensor Vehicle . . . . . . . . . . . . . . . . . . . . . . 55

3.2.4 Master Road Simulation . . . . . . . . . . . . . . . . . 56

3.2.5 Testing Modified Behavior of the Sensor Vehicle . . . . . . . 61

3.3 Wavelet Transforms . . . . . . . . . . . . . . . . . . . . 63

3.3.1 GNU Scientific Library . . . . . . . . . . . . . . . . . . 63

3.4 Machine Learning . . . . . . . . . . . . . . . . . 65

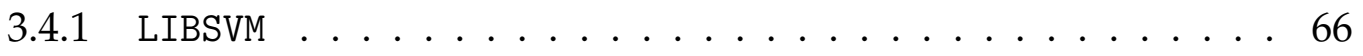


3.5 Signal Processing $\ldots \ldots \ldots \ldots \ldots$. . . . . . . . . . . 67

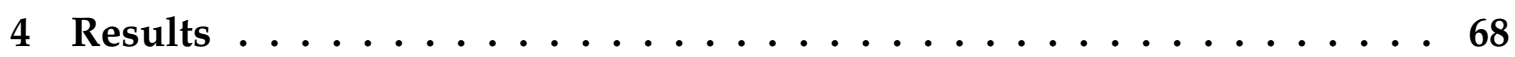

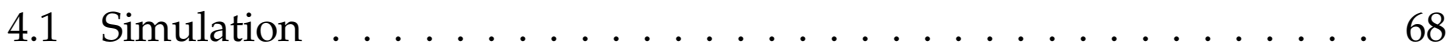

4.1.1 Explanation of Collected Data . . . . . . . . . . . . . . 69

4.1.2 General Characteristics of Collected Data . . . . . . . . . . 71

4.2 Wavelet Transform Performance . . . . . . . . . . . . . . . . 78

4.3 Support Vector Machine Calculation _ . . . . . . . . . . . 78

4.3.1 Support Vector Machine Calculation: First Iteration _ . . . . 79

4.3.2 Support Vector Machine Calculation: Second Iteration . . . . . 81

4.3.3 Support Vector Machine Calculation: Third Iteration . . . . . 83

4.3.4 Support Vector Machine Calculation: Fourth Iteration . . . . 86

4.4 Signal Processing $\ldots \ldots \ldots$. . . . . . . . . . . . . . . . 89

4.5 Summary . . . . . . . . . . . . . . . . 92

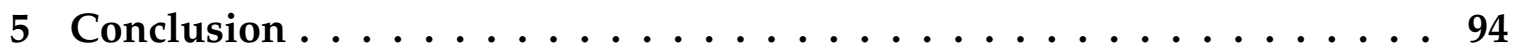

5.1 Summary . . . . . . . . . . . . . . . . . . 94

5.2 Opportunities to Extend this Work . . . . . . . . . . . . . 96

5.3 The Path to Implementing Intelligent Driver Feedback . . . . . . . 98

5.4 Opportunities after Implementation $\ldots \ldots \ldots$

5.5 Final Thoughts . . . . . . . . . . . . . . . . . . 100 Appendices

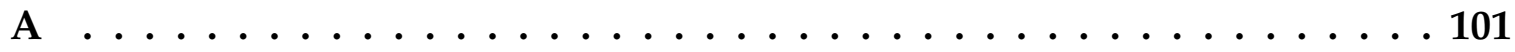

A.1 Computing Resources Used . . . . . . . . . . . . . . . . . . 101 
A.2 Software Used . . . . . . . . . . . . . . . . . . 101

A.3 Partial Compute Time Records . . . . . . . . . . . . . . . . . . 103

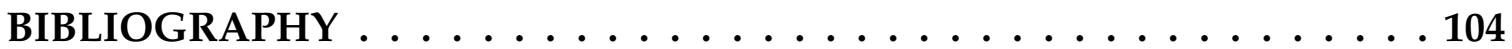




\section{LIST OF TABLES}

Table

Page

2.1 Model Parameters of the Intelligent Driver Model $\ldots \ldots$. . . . . . 35

3.1 Controlling Memory Parallel Reads/Writes . . . . . . . . . . . . 51

3.2 Parameters of the IDM Simulator . . . . . . . . . . . . . . 54

4.1 Results from Different IDM Simulations . . . . . . . . . . . . . 69

4.2 Descriptive Statistics from All Twelve Simulation Runs . . . . . . . 70

4.3 Best Accuracy Predicting Testing Data across All Values of $C$ and $\gamma$. . 80

4.4 Descriptive Statistics from Simulation Runs 1 and $2 \ldots \ldots$

4.5 Best Accuracy Predicting Testing Data across All Values of $C$ and $\gamma$. . 87

4.6 Results of Signal Noise Reduction . . . . . . . . . . . . . . . 90

5.1 Possible Improvements to this Approach . . . . . . . . . . . . . 97

A.1 Computing Resources Used for this Thesis . . . . . . . . . . 101

A.2 Partial Compute Time Records . . . . . . . . . . . . 103 


\section{LIST OF FIGURES}

Figure $\quad$ Page

1.1 Global Mean Surface Temperature Anomalies . . . . . . . . . . . . 5

1.2 Projected Temperature Change by $2100 \ldots \ldots \ldots \ldots$

2.1 IDM Equation of Desired Gap . . . . . . . . . . . . . . . 36

2.2 IDM Equation of Desired Acceleration . . . . . . . . . . . . 37

2.3 Example Support Vector Machines _ . . . . . . . . . . . . . . 40

3.1 High-level Flowchart of the Proposed IDM Simulation Project. . . . . 52

3.2 Display of the IDM simulator at clock step $0 \ldots \ldots \ldots \ldots$

3.3 Display of the IDM simulator at the beginning of a test run . . . . 57

3.4 Display of the IDM simulator during a test run $\ldots \ldots \ldots \ldots$

3.5 Display of the IDM simulator during a test run . . . . . . . . . . 59

3.6 Display of the IDM simulator during a test run . . . . . . . . . . 59

3.7 Display of the IDM simulator during a test run $\ldots \ldots \ldots \ldots$

3.8 Plot of Percent Road Occupancy versus Kinetic Intensity. . . . . . . 60

4.1 Plot of Percent Road Occupancy versus Percent Flow Change. . . . . 71

4.2 Histogram of Percent Flow Change. . . . . . . . . . . . . 72 
4.3 Occupancy as a Function of Time $\ldots \ldots \ldots \ldots \ldots \ldots$

4.4 Duration of Acceleration Suppression $\ldots \ldots \ldots$

4.5 Scatterplot of Flow versus Suppression Duration . . . . . . . . . . 74

4.6 Histogram of Acceleration Suppression Duration . . . . . . . . . . 75

4.7 Histogram of Event Flows $>1 \% \quad \ldots \ldots \ldots \ldots$

4.8 Scatterplot of Positive Results versus Suppression Duration . . . . . . 77

4.9 Example results from LIBSVM easy · py cross-validation script. . . . . . 82

4.10 Second iteration results from LIBSVM easy . py cross-validation script. 84

4.11 Third iteration results from LIBSVM easy · py cross-validation script. . 84

4.12 Heat map of results from fourth iteration of SVM calculation . . . . 88

4.13 SVM Accuracy given Smallest 480 of 512 Features Removed . . . . 90

4.14 SVM Accuracy given Smallest 448 of 512 Features Removed . . . . . 91

4.15 SVM Accuracy given Smallest 416 of 512 Features Removed . . . . . 91

4.16 SVM Accuracy given Smallest 384 of 512 Features Removed . . . . 92 


\title{
COUNTERACTING TRAFFIC CONGESTION USING INTELLIGENT DRIVER FEEDBACK
}

\author{
David K. Drum
}

Dr. Timothy C. Matisziw, Thesis Supervisor

\begin{abstract}
Traffic congestion is a daily occurrence in urban highway networks worldwide. It is not possible, however, for society to build its way out of congestion; rather, smarter roads and vehicles are needed. While the development of a smarter transportation system is underway, full implementation is years or decades from now. Yet, some of the sensing technology needed for smarter vehicles is already widely deployed in the form of smart phones. This thesis develops a novel method for recognizing traffic congestion using an artificially intelligent heuristic that could be implemented in a smart phone application or embedded system. Its goal is to provide intelligent feedback to a driver or autonomous vehicle control system to counteract stop-and-go traffic, a defining feature of urban highway congestion. Evaluation of the method indicates that a specific condition during stop-and-go traffic can be recognized accurately. A driver or control system acting upon feedback provided by the artificially intelligent system can improve traffic flow on the roadway by $1 \%$ to $3.5 \%$ over the course of the test duration.
\end{abstract}




\section{Chapter 1}

\section{Introduction}

In Tom Vanderbilt's popular 2008 book Traffic: Why We Drive the Way We Do (and What It Says About Us), he playfully cites a German advertisement: "You're not stuck in a traffic jam, you are the traffic jam" (Vanderbilt, 2008, p. 131). The ad works because it underscores a real truth about contemporary automotive culture. In trying to get somewhere, we are routinely stuck going nowhere.

The emphasis on the car as the primary means of transportation in America has produced some serious, if unintended, consequences: underfunded infrastructure and public transportation services, daily traffic congestion on urban freeways, and a looming environmental crisis, among others. The adoption of autonomous vehicles, though decades away, promises a new mobility-on-demand transportation paradigm in which the usage of each individual vehicle is maximized, dramatically dropping the number of vehicles in use and, as a result, reducing congestion and environmental impact. In the meantime, the appearance of connected vehicles sharing real-time information will empower drivers to make more informed choices 
about traffic conditions, improving traffic situations on small and large scales. Some of that real-time information can be sensed today by very simple instruments, such as sensors found in any of the modern smart phones used by millions of commuters on a daily basis. Given that the required sensing platform already exists, it's reasonable to expect that a smart phone application could detect certain traffic conditions in isolation and offer a driver useful information during their commute. Exploring that hypothesis and demonstrating that it can have a positive effect on overall traffic conditions is the subject of this thesis.

Much traffic congestion arises due to the way in which drivers respond to traffic conditions they encounter. Thus, providing drivers with feedback as to which of their responses may best alleviate congestion could yield positive impacts for an urban transportation system. This thesis investigates the extent to which a single vehicle can adjust its speed at the correct time to avoid amplification of or even dampen an upstream-traveling velocity shockwave for the benefit of the driver and the entire road system. To accomplish this, a traffic simulation framework is developed and implemented. Events collected from a simulation are then used to train a machine learning algorithm to detect opportunities for positive speed adjustments and provide feedback to the driver. Results indicate that critical speed adjustments and response times can be identified and, if better utilized by drivers, can have a positive impact on overall flow.

Traffic congestion in urban areas is known to be heavily influenced by the way in which individual drivers react to traffic conditions. When individuals overreact to or overcompensate for changing circumstances, a lack of capacity on the roadway can cause velocity shockwaves to radiate throughout the system. These 
shockwaves are a self-organizing, self-amplifying emergent behavior known as a traffic jam. If individuals were able to identify such velocity shockwaves and were able to respond in an appropriate manner, the waveforms could be mitigated, resulting in lower congestion. The ability to detect shockwaves and provide drivers with strategic responses will create destructive interference in the congested traffic velocity waveform, which will reduce the variance in drivers' speeds, harmonizing traffic flow. To this end, the focus of this thesis is on the design and implementation of a traffic simulation software system for: a) modeling triggered behavior change at the level of the individual vehicle, b) generating robust representations through a variety of wavelet transformations, and c) producing data suitable for input into a support vector machine (a type of Machine Learning algorithm) through which the intelligent feedback heuristic can be implemented.

The intelligent feedback heuristic proposed in this thesis could be deployed as a mobile application, leveraging the multitude of smart phones and other mobile communication devices utilized by urban commuters. Such an application would allow commuters to detect and appropriately respond to traffic conditions in such a way that could dramatically reduce the worst effects of congested urban freeway traffic. For example, an application could be designed to provide a driver with an alert (designed to minimize potential driver confusion and distraction) advising the driver to strategically modify their acceleration in order to promote smoother traffic flow. In time this heuristic could also be embedded into adaptive cruise control and autonomous driving systems, allowing vehicles to jointly work toward the systemic goal of speed harmonization. In addition, an intelligent technology such as this could offer opportunities for drivers and vehicles to share information 
about conditions amongst themselves and to coordinate their actions and amplify their effects. Anonymized data voluntarily shared by participants could provide summaries of current traffic conditions of use to public and private entities. Through the incremental accretion of individual actions, it would become possible to realize a system-wide effect. If one person can trigger a traffic jam, one person may indeed be able to defuse it.

\subsection{The Automobile's Unintended Consequences}

The vision of personal mobility that became so central to the American Dream in the postwar 20th century is exacting a high societal cost given that the resulting congestion has not been addressed in a comprehensive, coordinated way. Personal motor vehicle transportation is the default transportation mode choice-tacitly required-in much of the United States, particularly the vast expanses of suburbia surrounding major cities. In the process of population shifting from the cities to the suburbs, America built nearly four million miles of roads (NationalAtlas.gov, 2013) to facilitate urban movement. At the same time, population growth nationwide combined with increasing use of personal automobiles has created additional demand for new roads and increased access, pitting expansion against maintenance.

Americans aspire to the egalitarian ideal of public roads, but behave quite differently, chafing at control systems like ramp meters (Levinson and Zhang, 2006), rejecting needed changes in traffic management systems such as congestion pricing and toll roads (Schaller, 2010), eschewing alternative transportation mode choices and relegating neglected public transportation systems to the bottom of the heap, 


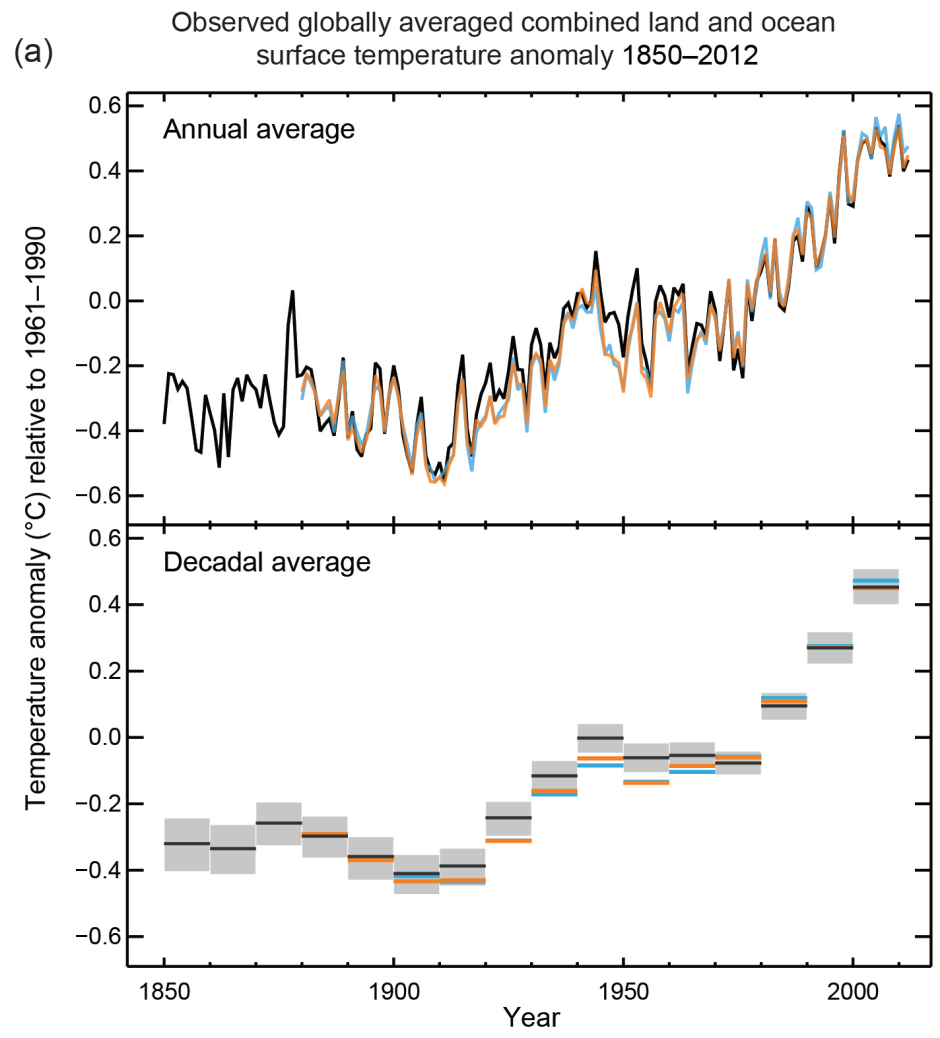

(b)

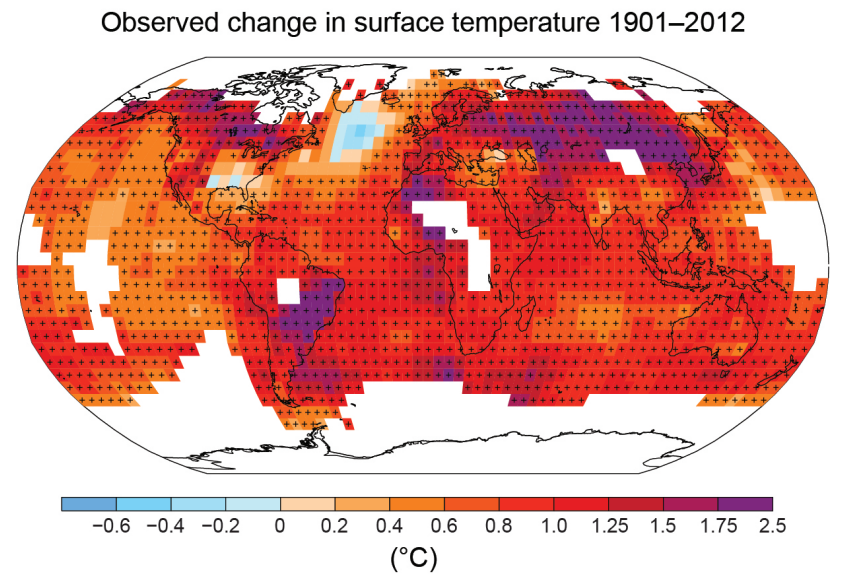

Figure 1.1: (a) Observed annual and decadal global mean surface temperature anomalies from 1850 to 2012 and (b) map of the observed surface temperature change from 1901 to 2012 (Stocker et al., 2013, reproduced with permission) 


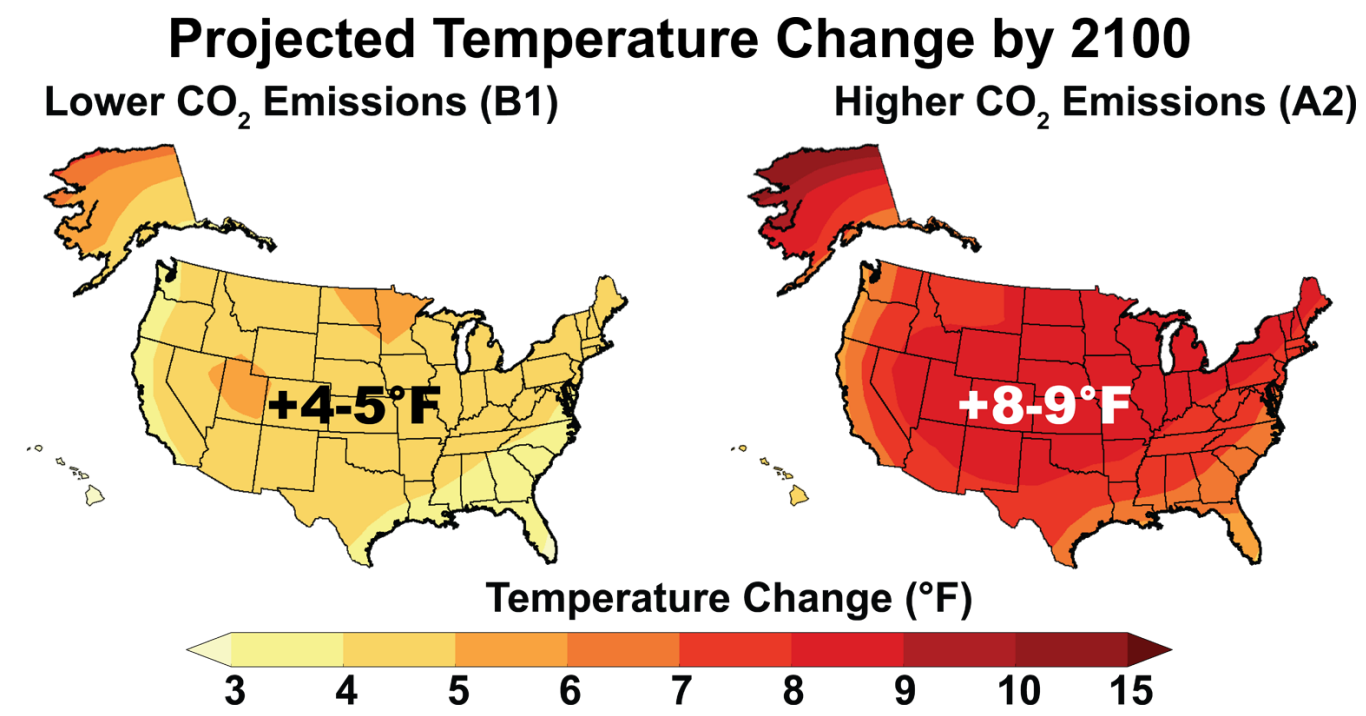

Figure 1.2: Projected Temperature Change by 2100 (Melillo et al., 2014, in the public domain)

the fate of people who cannot drive (Glaeser et al., 2008; Sanchez, 2008). As a result, urban, and even suburban, traffic congestion continues to worsen, offset briefly by the recent economic recession (Schrank et al., 2012). More sobering, America's foreign policy has been heavily influenced by its dependence on low cost fuel (Yergin, 2011). Moreover, human-generated climate change is now, and has been for some time, an established scientific fact (Figure 1.1) (Pachauri and Intergovernmental Panel on Climate Change, 2008; Cook et al., 2013; Stocker et al., 2013; American Association for the Advancement of Science, 2014) with consequences not to be realized for decades (Figure 1.2). The transportation sector, via greenhouse-gas emissions, has been identified as a significant contributor to this problem (Barth and Boriboonsomsin, 2008, 2009). 


\subsection{The Need for Autonomous Vehicles}

Autonomous vehicles are vehicles which can move "without the active control or monitoring of a human operator" (Nevada State Legislature, 2013) using a combination of active and passive environmental sensors, digital maps and databases about the geography in which they operate, and robotic control systems. A crucial, long-term benefit to be realized by autonomous vehicles is the significant reduction in the number of vehicles operating on the roads. Most personal vehicles sit idle most of the day, resulting in a fleet utilization percentage around $4 \%$ (Chesbrough, 2010). Even the most basic estimates suggest that there are sixteen to twenty times as many vehicles in existence as are actually needed, but even a conservative estimate is still three times too many vehicles (Spieser et al. (2014) in Meyer and Beiker, 2014, in press).

Enabling vehicles to intelligently reposition themselves for other purposes could have a variety of positive effects. From a transportation perspective, it would reduce the vast amounts of land dedicated to surface parking, reduce the need for parking structures, end congestion, and perhaps most importantly, reduce the need for dead mileage, endless circling for a convenient parking space (Shoup et al., 2005) and backhauls, return trips by a driver without passengers or cargo (McKinnon and Edwards, 2010). It would also allow alternative, environmentally friendly engine fuels not as feasible in consumer markets (electric and hydrogen) to replace gasoline and diesel fuels in fleets operated by new, autonomous mobilityon-demand services (Riley, 1994). Another long-term benefit would be a reduction in accidents due to human error, perhaps by as much as an order of magnitude (Maddox, 2012). The autonomous vehicle has been called an idea that will upend 
transportation, and this thesis proposes methods and techniques in support of this emerging technology.

\subsection{The State Of The Autonomous Vehicle}

Science-fiction writer William Gibson has said, “The future is already here. It's just not very evenly distributed" (Gibson, 1999). His comment is particularly applicable to the case of autonomous vehicles.

To fully explain the significance of this research and to appreciate its place in the unfinished puzzle that is autonomous vehicle technology, it is necessary to first review available driver-assistance technologies. Currently in the automotive industry, standard options such as electro-hydraulic "power" steering and brakes are available for production vehicles. Almost all models have the option to be equipped with cruise control, rear hazard alerts, anti-lock brakes, and electronic stability/traction control, all of which begin to blur the line of control between driver and vehicle (Leen and Heffernan, 2002). More advanced driver assistance and enhancement systems are currently limited to luxury vehicles, and include adaptive cruise control, lane departure warnings, forward collision warnings, side and rear hazard cameras, automated lane changes, and parking assistance (IndustryARC, 2013). All of these advanced systems are dependent on ever more complex passive and active sensors and algorithms and are slowly becoming available on more mainstream vehicle models.

Nearly every large American, European, and Japanese automobile manufacturer is pursuing an autonomous vehicle research project at this time (Knight, 2013). Un- 
der development are capabilities that will facilitate semi-autonomous and fully autonomous vehicles. Some near-term capabilities targeted toward semi-autonomous driving by production vehicles are the abilities to car-follow (longitudinal and lateral control) and drive fully autonomously on highway stretches for a short period of time (General Motors, 2013; Mercedes-Benz USA, 2013). Beyond that lie the research projects pursuing comprehensive autonomous driving, featuring a multitude of sensors and dedicated computing resources. These projects are not geared toward production models yet, with their trunks full of computer servers or their $\$ 70,000$ roof-mounted LiDAR units, in the case of Google's effort (Silberg and Wallace, 2012). However, if the past is any predictor of the future, sensors and computers will shrink and drop in cost to the point where they are implemented at the fleet level.

A related effort currently underway is what has been termed the connected vehicle. The connected vehicle refers to the effort to improve the safety, mobility, and environmental impact of vehicles through vehicle-to-vehicle and vehicle-toinfrastructure communications (U.S. DoT RITA ITS Joint Program Office, 2014a). The safety aspect will be achieved by allowing nearby vehicles to report conditions to each other. The mobility and environmental impact aspects will be achieved through "big data" aggregation and reporting, helping commuters and transportation personnel make better use of the finite road resource. One particular way the mobility and environmental impact goals of connected vehicles will be achieved is through the concept of cooperative adaptive cruise control, where a group of vehicles with similar routes on a roadway will match their velocities and form a 
flotilla, both increasing the upper limit of vehicle density on roads and achieving higher fuel economy through aerodynamic effects (Shladover, 1991).

It is natural to envision that autonomous vehicles will also be connected vehicles because the goals of both efforts overlap. Additionally, connected vehicle technology will complement the capabilities of autonomous vehicle technology and mitigate some of its weaknesses, specifically, the limited sensing range of autonomous vehicles. For the purposes of this thesis, an autonomous vehicle will be assumed to also be a connected vehicle, able to communicate with various vehicle information resources.

We are some decades away from the end goal of fewer vehicles on the road, most of which will operate autonomously at much higher utilization rates. Prior to that will be a period of transition where increasing numbers of fully autonomous and semi-autonomous vehicles (specifically, those with adaptive or cooperative adaptive cruise control) capable of sensing traffic congestion, have the capacity to make small adjustments that would incrementally work against the symptoms of congestion. The physical platform required for sensing those conditions is quite modest, namely, a device for detecting axial movement (i.e. accelerometer) and a devices for identifying the location of a vehicle (i.e. GPS receiver). In fact, everything required to sense the relevant conditions, process the information, and advise the driver can be found in a modern smart phone. This opens the door to providing more intelligent, real-time decision support to drivers and cars smarter, right now, while we wait for the future to arrive. 


\subsection{Personal Motivations}

The automobile and the Interstate Highway System have had significant impacts on my life. My grandfather was the first truck driver hired at Potashnick Construction Company in Sikeston, Missouri. He spent much of his career driving low-boy, flatbed trucks transporting heavy equipment for large construction projects (including dozens of Interstates and the Ohio Turnpike) throughout the United States from the 1950s through 1970s. My father, while a high school and college student, worked as a surveyor's assistant during the summer on Western Kentucky Parkway survey crews.

Growing up, I lived in suburban South St. Louis County. My father worked in downtown St. Louis. To avoid the morning and afternoon rush hours, he kept a 7:00 a.m.-3:00 p.m. workday. Throughout my childhood it was clear this was why we kept the household schedule we did. It was the first major but indirect effect that urban freeway congestion had on my life.

At the same time, our summer vacations were all based around Interstate road trips, commonly Florida and elsewhere in the South, Kentucky, Kansas, and Colorado, camping along the way. I developed a taste for the open road. While in college at the University of Missouri-Columbia in the late 1980s and early 1990s, I would return to St. Louis for the occasional weekend home. While driving eastbound on U.S. Highway 40 through St. Charles County on Friday afternoons, trip after trip I would see westbound traffic backed up from the MO-94 exit for miles, across the Daniel Boone Bridge, through Chesterfield and West St. Louis County, miles and miles of stop-and-go traffic. I resolved not to move to an area after college where I would have to commute like that. 
I continued making road trips as a young adult: Florida and the South, multiple times; Route 66; Arizona, California, and Utah. And, memorably, New England into Canada, where I had no idea what the signs telling drivers to keep two chevrons between vehicles meant while driving through Toronto. Similarly impressive was the adrenaline surge of driving into Los Angeles, pacing seasoned commuters right up to the moment traffic came to a complete stop.

It was on one of these road trips that I thought "What if cars could drive themselves?" That thought experiment provided mental fodder for many miles

and years to come: sensors; vehicle-to-vehicle communication; the possibility for intersection-scheduling algorithms; mobility-on-demand, parcel, and shipping services; routing databases and the commercial interests that would exert influence over them; the ability to hack vehicles, for good or ill; the value of open systems; the chance to end congestion.

Some of this science fiction has come to pass, through the DARPA Grand Challenges, the work of Brad Templeton, Sebastian Thrun and Stanford, Google, and many others around the world. Yet the bulk of the work toward fully autonomous vehicles still lies ahead, and that was a major factor in my decision to pursue a Master's degree in Transportation Engineering.

\subsection{Organization of this Thesis}

The remainder of this thesis is organized as follows. First, a review of the literature will discuss the nature and impact of urban traffic congestion; the availability of driver assistance systems; and the creation of an intelligent driver feedback system. 
Second, a discussion of the methods used to develop the intelligent driver feedback system, including traffic modeling and machine learning, is presented. Third, the results of traffic modeling and machine learning are presented and analyzed. Finally, conclusions are drawn and opportunities for extending the work are described. 


\section{Chapter 2}

\section{Literature Review}

The following literature review is organized into three major sections relevant to this thesis research: 1) Congestion, 2) Driver Assistance Feedback Systems, and 3) Creating a Feedback Heuristic.

\subsection{Congestion}

One of the biggest problems autonomous vehicle technology could help mitigate is the problem of congestion. The phenomenon of congestion on urban freeway systems is familiar to drivers, chronic around the world (Downs, 2004; Elkatsha, 2013), and a major focus of study: between 2003 and 2012, 6.85\% of all articles cataloged in the Transportation Research Board's Transportation Research Integrated Database (TRID) are tagged with the keyword "congestion" (Transportation Research Board, 2014). Congestion is commonly characterized as a breakdown in traffic flow, flow being the number of vehicles passing a given point during a specific period of time. 
Flow breakdown occurs after a capacity threshold has been exceeded and can occur anywhere along sections of highway where the influences of fixed bottlenecks and traffic stream dynamics combine to precipitate capacity changes (Öğüt and Banks, 2005). Capacity changes occur when traffic flow increases beyond road capacity or road capacity decreases below traffic flow. These changes can be attributed to bottlenecks (lane unavailability due to accident, interchanges, etc.); increases in traffic flow beyond capacity; or sometimes from transient disturbances, such as individual driver behavior, that the system is unable to absorb. When the capacity changes cross a non-deterministic threshold, congestion will result (Öğüt and Banks, 2005).

The onset of congestion may be broadly described as follows: the traffic stream degrades from free flow, where vehicles have few interactions (individual vehicles' accelerations and decelerations do not affect other vehicles); through synchronized flow, where vehicles interact but maintain homogeneous velocities and are able to absorb perturbations; to congestion. Symptoms of congestion are expressed as flow reduction and velocity shockwaves propagating against the flow of traffic (upstream) from the disturbance (Cassidy and Bertini, 1999; Wilson, 2008; Orosz et al., 2009; Yeon et al., 2009). At the level of the individual driver, congestion is characterized as either prolonged cycles of speed changes: stop-and-go traffic (also referred to as slow-and-go) or, in the worst case, complete traffic paralysis. Congestion which cannot be traced to a bottleneck is often referred to as a selforganized or "phantom" jam and occurs when the critical threshold of vehicle density is exceeded (Sugiyamal et al., 2008). Jerath and Brennan (2012) show both that the traffic density (vehicles per unit distance) at which congestion criticality 
is reached is higher in a fleet of adaptive cruise control (ACC) equipped vehicles and that adding non-ACC vehicles to the fleet, where humans are responsible for longitudinal control (acceleration and deceleration), increases the likelihood of a phantom jam. One may therefore conclude that driver reaction time and subsequent overreaction to a braking stimulus is a key contributing factor to triggering a phantom jam (Zielke et al., 2008). The speed of individual cars varies widely within congested conditions, but as a whole, traffic moves at a rate substantially lower than at maximum flow (6\%, Hall and Agyemang-Duah, 1991), (10\%, Cassidy and Bertini, 1999), (20\%, Treiber et al., 2000). Additionally, a statistical analysis of four months

of empirical data revealed that the U.S. Department of Transportation Highway Capacity Manual (2011) overestimated flow within flow breakdown conditions and at leaving such conditions (Yeon et al., 2009). It is worth considering whether drivers have a more difficult time adapting to flow breakdown conditions than is generally recognized (Levinson et al., 2004), and if feedback can improve driver performance and therefore overall conditions.

\subsubsection{Impacts of Congestion}

The magnitude of traffic congestion is enormous and growing. A study of five years of data from California freeways shows drivers spending $20 \%$ of their time in congestion (Varaiya, 2005). The INRIX Gridlock Index of the 10 largest cities in the U.S. for October 2012 reported trips at 17.6\% longer than necessary (INRIX, 2012). Gibbens and Saatci (2008) evaluated 247 weekdays in 2003 from the M25 roadway in London and showed that the median delay experienced by commuters is $30 \%$ of the median hours traveled. The Texas Transportation Institute has reported on 
the growing problem of congestion over the past 30 years. Commuters now waste approximately $20 \%$ of their commute in congested traffic (Schrank et al., 2012). The American Community Survey \#15 from the U. S. Census Bureau (2011) reported $76 \%$ of workers (105.5 million people) in the United States driving alone on their daily commute. Traffic congestion is therefore a significant cost in the United States to individual drivers ( $\$ 818$ and 38 hours average delay annually) and society as a whole (\$121 billion and 5.5 billion hours delay annually) (Schrank et al., 2012, 2011 figures).

All drivers experience higher stress and aggression in high-congestion situations than low-congestion situations (Hennessy and Wiesenthal, 1999). However, the effects of stress are inconsistent and vary from driver to driver. As a whole, a driver's susceptibility to congestion related stress has been expressed in the literature using an impedance metaphor (Koslowsky et al., 1995; Novaco and Gonzalez, 2009; Amichai-Hamburger, 2009). Individuals appear to have different impedance levels with respect to commuting in general and congestion in particular. For those who are susceptible, however, the different effects make for a very unpleasant list: physical problems include elevated cardiac activity and stress hormone levels, physical pain, especially back problems, digestive disorders, and even stroke and cancer; psychological problems in the workplace include low performance, missed work, and job turnover. Additionally, traffic congestion has been associated with increased incidence of accidents resulting in death or serious injury (Wang et al., 2013). Congestion therefore can be viewed as exacting a significant human cost.

The Texas Transportation Institute has published findings that fifty-six billion pounds (25.4 million metric tons) of carbon dioxide were released in 2011 due to 
urban roadway transportation congestion (Schrank et al., 2012). Using the U.S. Energy Information Administration figure of 1,519 million metric tons emitted by the transportation sector in 2011, approximately $1.7 \%$ of all transportation-related greenhouse gas emissions were due to congestion.

\subsubsection{Solutions}

Solutions to congestion are elusive, but the need for them is great (Schrank et al., 2012). The traditional approach to solving roadway congestion has been to build new roads and add more lanes to existing roads, yet it is a trivial economics exercise to show that increasing supply increases demand. As a result, adding road capacity does nothing to address congestion and actually worsens the situation; known as the Lewis-Mogridge Position and Downs-Thomson or Pigou-Knight-Downs Paradox (Lewis, 1977; Mogridge, 1990; Arnott and Small, 1994). Newer, systemic approaches based on implementable technology such as ramp metering, variable speed limits, dynamic messaging systems, and congestion pricing are deployed in many urban centers around the world, but are designed to address the macroscopic performance of the system (Shibata and French, 1997; Chen et al., 1999). Even with these measures, traffic systems are unable to quickly recover high-flow conditions following a perturbance (Gibbens and Kelly, 2011).

At the leading edge of automobile technology are autonomous vehicle projects by Audi, BMW, Daimler, Ford, GM, Google, Nissan, Toyota, Volvo, and others that aim to reduce human driver fallibility (Knight, 2013), but general availability and widespread adoption is many years away not only due to a patchwork of regulatory frameworks (Kalra et al., 2009; Lassa, 2013) but also because the current 
cost of technology for a fully autonomous vehicle significantly exceeds the cost of an average vehicle (Rebsamen et al., 2012; Litman, 2013). Additionally, autonomous vehicles in a heterogeneous fleet will only be able to drive as efficiently as (though more safely than) the human-controlled vehicles around them (Jerath and Brennan, 2012).

A related approach to solving congestion at the microscopic scale is the concept of connected vehicles, that is, human-operated vehicles which can communicate information about their state to nearby vehicles and roadside infrastructure (U.S. DoT RITA ITS Joint Program Office, 2014a). Connected vehicles are expected to increase driver awareness of their immediate surroundings, but the effect of that awareness remains a topic of research by the US DOT RITA Connected Vehicle Safety Pilot (U.S. DoT RITA ITS Joint Program Office, 2014b). Cooperative Adaptive Cruise Control based on simulated connected vehicle technology has been shown to counteract stop-and-go shockwaves in urban freeway traffic models, reducing emissions, noise, and vehicle wear by decreasing the kinetic intensity of vehicles (Ma et al., 2012). However, that particular computer model required a very high percentage of adoption in order to realize overall improved flow and environmental benefit (Ma et al., 2012). This requirement directly contradicts conclusions in other research into the effects of Adaptive Cruise Control on congestion (Section 2.2.2), indicating a need for further research. Regardless, most vehicles lack these embedded systems, and aftermarket solutions do not exist due to their nature and risk of liability (Kalra et al., 2009).

There appears to be a lack of practical solutions to address congestion at the microscopic scale. This thesis proposes one solution for filling that gap. Though 
the mass-adoption of autonomous vehicles is not a feasible alternative at this time, driver-assistance technologies, like the heuristic model developed later in this paper, can serve as an evolutionary step toward that reality.

\subsubsection{Congestion Shockwaves And Traffic Oscillations}

A traffic shockwave is defined as the point of transition from one state of traffic flow to another (Öğüt and Banks, 2005). A vehicle on an urban highway may travel at the speed limit in uncongested conditions (free flow), having little interaction with surrounding vehicles (in particular, the vehicle directly ahead), until the vehicle catches up to a slower vehicle. The position of the vehicle when it is obliged to brake in order to maintain a safe following distance is the position of the traffic shockwave. Shockwaves almost always travel upstream, passing from leading car to following car (Cassidy and Bertini, 1999). This is easily demonstrated in that a leading vehicle can force a following vehicle to brake, but a following vehicle cannot force a leading vehicle to accelerate. Traffic oscillations are cyclical changes in vehicle streams' speed over time (Zielke et al., 2008) as vehicles react to passing through shockwaves.

One of the tell-tale signs of congestion is stop-and-go traffic along sections of roadway. The oscillation of vehicle speeds in stop-and-go traffic is a symptom of string instability within a platoon of vehicles (Kesting and Treiber, 2008). Traffic oscillations may be unavoidably linked to human driver behavior (Igarashi et al., 2001; Kesting et al., 2008a; Wilson, 2008; Orosz et al., 2009), for example, anticipation and overreaction (Yeo and Skabardonis, 2009) or aggressiveness and timidity (Laval and Leclercq, 2010). 
Zheng et al. (2011) define an oscillation as deceleration and subsequent acceleration. Zielke et al. (2008) define a traffic oscillation more formally by citing cyclical traffic speed, congested conditions, and upstream propagation. Once in an oscillation pattern, a driver is then obliged to brake and accelerate cyclically until her destination is reached or flow recovers. The repetition of braking and accelerating are mutually canceling events, and therefore entirely wasteful.

Additionally, the performance characteristics of the vehicle's internal combustion engine must be taken into account; tailpipe emissions are greatly increased during high acceleration (Coelho, 2005; Servin et al., 2006; Coelho et al., 2009). Barth and Boriboonsomsin (2008) examine congestion and its carbon footprint and recommend macroscopic traffic-smoothing techniques through variable speed limits and intelligent speed adaptation, incurring a 7.0-12.0\% (typical) to $45.0 \%$ (ideal) reduction in carbon-dioxide emissions. An empirical study of 90 drivers "concluded that a modest saving in fuel can be achieved by maintaining a steady pace and leaving substantial headway" (Jones and Appleby, 1978, p. 540). Similarly, Evans (1979) and Barkenbus (2010) recommend anticipating conditions ahead and minimizing braking and acceleration to reduce fuel consumption.

These recommendations foreshadow the concept of a minimally consumptive (fuel, brake pad, etc.) driving behavior currently called "eco-driving". Eco-driving training at Mercedes-Benz was found to result in a 15.0\% savings based only on "accurate information and feedback" (van der Voort, 1997). The International Transport Forum concluded that eco-driving could achieve 10.0\% fuel savings (International Transport Forum and International Energy Agency, 2007). In another study (Frey et al., 2003), short-term events (specifically high acceleration) were found to con- 
tribute significantly to overall emissions and the authors advise addressing factors that lead to their occurrence.

In order to approximate desirable driving behaviors numerically, a mathematical solution could be beneficial. O'Keefe et al. (2007) define kinetic intensity (p. 5) as the ratio of characteristic acceleration (a measure of a cycle's acceleration and grade intensity, p. 4) to the square of aerodynamic speed (p. 4). A vehicle will produce a higher kinetic intensity value during hard acceleration and deceleration actions, though this value will decrease as the vehicle's speed increases. Smooth driving with gentle acceleration and deceleration will produce a lower kinetic intensity. Kinetic intensity is therefore a quantitative measure of eco-driving behaviors which will later be used to gauge individual driving actions and overall trip impact.

\subsubsection{Mathematical Solubility}

Colombo and Groli (2004) use calculus of variations (Gelfand and Fomin, 2000) to prove the existence of an average traffic density that will minimize oscillations in the classic Lighthill-Whitham-Richards (Lighthill and Whitham, 1955; Richards, 1956) and related macroscopic traffic flow models, resulting in more fluent traffic. This is accomplished, in part, by selecting a Lipschitz function that minimizes "the (weighted) total variation of the car speed" (Colombo and Groli, 2004, p. 701). Briefly, a Lipschitz function is constrained in how fast its value can change. While that mathematical construct will not be explored here, it establishes that fluent traffic is dependent on achieving an average traffic density, which in turn is dependent on minimizing the total variation of vehicle speeds. This point will be revisited later in the thesis. 


\subsection{Driver Assistance and Feedback Systems}

Having established that a particular set of general driving behaviors can mitigate traffic congestion, the question becomes one of determining if there are actions drivers can take at specific times to mitigate congestion and how to motivate the driver to behave in that manner. Driver-assistance systems such as dynamic messaging signs, congestion maps, and personal routing devices provide valuable information to individual drivers that may affect their trip planning but do not generally contribute to helping them become better drivers. Currently, several aftermarket products exist that provide drivers with additional dashboard-style information calculated from data obtained through the vehicle's On-Board Diagnostics (OBD-II) port. ScanGauge and UltraGauge are dedicated hardware components that include function-specific displays reporting data from the engine computer, including engine maintenance codes, to the driver (ScanGauge, 2013; UltraGauge, 2014). Being firmware-based solutions, their feature set and analysis capability is limited. Other approaches use a driver's smart phone as the compute and display platform. Automatic, OBD Mileage, DrivingStyles OBD-II Behavior, and others take a hybrid approach, connecting via the Bluetooth wireless standard to an adapter connected to the OBD-II port to provide feedback about rough driving behaviors (strong acceleration, speeding, hard braking), summarize and quantify these events to motivate more environmentally conscious driving behaviors, tracks trips and gas mileage, initiate crash alerts, interpret engine maintenance codes, and provide directions to where the car is parked (Automatic, 2013; Universitat Politècnica de València and Grupo de Redes de Computadores, 2014; Findra Studio, 2014; Ishida $R \& D, 2013)$. 
These products calculate and report values of importance to eco-drivers such as fuel consumption (instantaneous miles per gallon). While a knowledgeable driver is able to consciously choose to adopt an eco-driving style, many drivers are unaware of eco-driving techniques, need external reinforcement to maintain those behaviors, or would welcome assistance, particularly during stressful, congested traffic situations.

According to the definitive study by Zielke et al. (2008) upstream propagation is an integral component of oscillating traffic. The effects of a drivers' actions in congested traffic are propagated from leading to following (upstream) vehicles. These actions can be characterized as having a positive or negative effect on traffic flow. Having established that maximizing traffic flow is dependent on minimizing the variation of velocities (Section 2.1.4), smooth driving behaviors, including ecodriving behaviors and the velocity adjustments recommended by a smart phone application that could be developed from this thesis, can be said to have a positive effect on traffic flow. As such, assisting drivers to change their driving styles and providing "accurate and frequent" (van der Voort, 1997, p. 32) feedback to maintain the new behaviors over time can have immediate and widespread positive economic and environmental impacts for any driver in any vehicle (Seligman, 1978; van der Voort, 1997; van der Voort et al., 2001; Barkenbus, 2010). However, as these are optional behavioral changes, the percentage of drivers adopting these behaviors is likely to remain small. It then remains to be seen if the actions of a small percentage of drivers can have an overall positive impact on the fleet. 


\subsubsection{Safety}

Zheng et al. (2010) reviews three years of loop detector and crash data for a segment of Interstate highway north of Portland, Oregon. Analysis of their data indicates that the crash occurrence probability increases by $8.4 \%$ per 5 -mile-per-hour increase in the standard deviation of the fleet speed distribution during congested traffic conditions. Zhang et al. (2011) finds that drivers in stop-and-go traffic can react to relative speed stimulus from as much as the third car ahead and that drivers are more aware of speed than space differences. Bauer et al. (2009) describes Variable Message Sign systems in Germany and show that speed harmonization leads to a $10-30 \%$ drop in accident rates. Riener and Ferscha (2009) model vehicles with time-to-collision feedback to the driver and find that throughput on the simulated road increases and trip time decreases across a range of vehicle densities. Islam et al. (2012) studies loop detector data from a stretch of roadway over a period of sixteen months in Edmonton, Canada and find that higher speeds, higher standard deviation of speeds, higher coefficient of variation of speeds, and average occupancy are all indicators of the likelihood of a crash. Vadde et al. (2012) models a stretch of US Highway 90 in San Antonio, Texas where an Active Traffic Management system is proposed and find that speed harmonization results in $14 \%$ fewer crashes. Harmonizing vehicles' velocities so that all vehicles along a stretch of roadway are traveling at approximately the same speed as a means to reduce the likelihood and severity of freeway accidents and improve flow and capacity is an established method with a proven track record. 


\subsubsection{Adaptive Cruise Control}

Advanced driver assistance systems (adaptive cruise control, lane departure warning, etc.) are generally only found in luxury vehicles and serve as a driver convenience rather than driver enhancement (Nirschl (2007) in Smith and Salvendy, 2007). Research has shown that appropriately calibrated Adaptive Cruise Control can reduce traffic congestion on a roadway even when only a small percentage of the fleet is so equipped (Zhou and Peng, 2005; Kesting et al., 2007b, 2008b, 2010; Orosz et al., 2010). Adaptive Cruise Control improves traffic conditions by delaying the onset of congestion and reducing the maximum queue length behind a bottleneck, which allows the congestion to dissipate earlier (Kesting et al., 2007b). Yet the fleet penetration of Adaptive Cruise Control worldwide is projected to reach only 5.2 million vehicles by 2015 (Global Industry Analysts, Inc., 2012), a small fraction of the one billion vehicles currently on the road (Sousanis, 2011). Additionally, in evaluating the tendency of drivers to lose attention and situational awareness when using Cruise Control and Adaptive Cruise Control, Vollrath et al. (2011) observed that "it would make more sense to develop warning and intervention systems which support the driver" (Parasuraman and Riley, 1997; Young and Stanton, 2002; Vollrath et al., 2011). The heuristic proposed in this thesis will initially be implemented as if to be deployed as a driver-assistance tool via a smart-phone application but could easily extended to be embedded in an Adaptive Cruise Control system. As vehicles become more fully autonomous, the driver distraction issue noted by Vollrath et al. (2011) should become less of an issue. 


\subsubsection{Smart Phones}

A smart phone is generally defined as a mobile computer capable of running thirdparty applications and featuring a variety of embedded sensors and antennae, allowing for communication on modern wireless networks (Lane et al., 2010). The adoption of smart phones has outpaced any previous consumer technology (DeGusta, 2012; McGrath, 2013). In the United States, $56 \%$ of the adult population owns a smart phone as of 2013 and is even higher among two-thirds of the workforce (ages 18-29, 66\%; ages 30-49, 59\%) (Smith, 2012). Smart phones represented 77\% of the growth in mobile devices worldwide in 2013 (Cisco, 2014). As such, availability of a smart phone as a platform for the development of an intelligent driver feedback application designed to utilize the heuristic developed here is seen as an opportunity more than an obstacle.

\section{Smart Phone Applications}

The current generation of smart phones include a variety of physical sensors. Smart phone manufacturers and third-party application-developer communities have written many applications that utilize these sensors. There has been a general recognition of opportunities for constructive use of smart phone applications in transportation. Other papers describe research applying smart phones' accelerometers and GPS receivers to accurately classify driving behavior (Johnson and Trivedi, 2011), detecting accidents (White et al., 2011), detecting drunk driving (Dai et al., 2010), providing driver feedback regarding a variety of conditions (Fazeen et al., 2012), providing warning of upcoming stop signs (Tucker et al., 2012), and collecting data about road conditions (Bhoraskar et al., 2012). 
A handful of smart phone eco-driving applications have been developed for the general public. Hunter Research and Technology's greenMeter (Hunter Research \& Technology, 2014) uses the iPhone accelerometer to gather data and infer eco-driving behaviors, providing a variety of options for data display similar to ScanGauge and UltraGauge (Section 2.2). No conclusion can be reached about its efficacy based on the application's reviews, though the web site estimates that the application has allowed drivers to save two million gallons of gasoline in the first year of its availability. However, the application's web site does not document the collection of these data. The application is available for US\$5.99 (April, 2014) on the U.S. Apple iTunes Store, but has not been updated since August, 2009. DriveGain (DriveGain Limited, 2011) provides similar functionality and outputs and purports to allow drivers to save US\$210 per year on fuel. Unlike greenMeter, it explicitly uses the iPhone's GPS receiver. The application is available in free (with optional inapplication purchases) or US\$6.99 versions (April, 2014). No equivalent non-OBD-II smart phone applications were found for the Google Android platform. Other smart phone platforms were not surveyed due to their low market penetration. A smart phone application based on the intelligent feedback heuristic developed in this thesis would differ from existing applications in the following ways:

1. It will detect a particular set of traffic conditions and recommend a particular behavior, rather than provide general feedback on general driving behaviors;

2. It is meant only for use in metropolitan and urban freeway settings, rather than in all traffic situations;

3. While it will continuously monitor traffic conditions and driver response, it will not provide continuous display or feedback, only providing decision support as necessary; 
4. When it provides feedback to the driver, it will be able to sense whether or not its recommendation was followed;

5. It will be able to detect the duration of time between providing feedback to the driver and the vehicle braking, allowing it to calculate its own accuracy, whether or not the driver followed the feedback;

6. If it detects that its feedback was followed, it will be able to estimate savings incurred by the driver and by following vehicles.

In a traffic context, the most important information a smart phone can give a driver includes the following:

- Conditions ahead (requires external information; cf. Google Maps, Apple Maps, Waze, etc.);

- Characterization of traffic conditions (Bhoraskar et al., 2012);

- Accident detection (White et al., 2011);

- Indicators of erratic driving (Dai et al., 2010; Johnson and Trivedi, 2011; Fazeen et al., 2012);

- Indication of speed oscillation (independent detection).

Zhang (1999) found traffic oscillation frequencies to average around one minute, which is a reasonable time window for a smart phone application to track data for processing. Chen et al. (2012) report oscillation periods approaching a lower bound in the range of 2-3 minutes. Kesting et al. (2008a) in Uhrmacher and Weyns (2009) list the period of stop-and-go waves as being on the order of ten minutes, while Laval and Leclercq (2010) report others' findings of longer periods of up to 15 minutes. If indeed such traffic phenomenon are on the order of 10-15 minute intervals, then the impact of a smart phone decision support device on driver behavior may be limited. Further research is clearly required to investigate this issue. 


\section{Sensing and Locating}

The 3G Partnership Project specification 25.305 lists standardized methods for a smart phone to determine its position, including Global Positioning Systems (GPS) (3rd Generation Partnership Project, 2013). The GPS receiver chip is among the highest power-consuming environmental sensors on a mobile device (Mohan et al., 2008; Ben Abdesslem et al., 2009; Dai et al., 2010; Lu et al., 2010; Tucker et al., 2012). Conversely, the accelerometer is its lowest power-consuming environmental sensor (Ben Abdesslem et al., 2009), making it practical for continuous, background use. By combining continuous monitoring of the battery-friendly accelerometer with strategic sampling of the power-hungry GPS receiver, a continuous location can be approximated within the error range of the Global Positioning System (Ben Abdesslem et al., 2009), maximizing location data availability while minimizing power consumption. The Jigsaw continuous sensing engine for smart phones also seeks to maximize location accuracy while minimizing power consumption ( $\mathrm{Lu}$ et al., 2010). It is not unrealistic to propose implementing a smart phone application intended to be active during a commute that depends on these two device sensors, assuming that sufficient coverage by navigation satellites exists.

\section{Human Factors}

Driver distraction from electronic devices (among many other in-vehicle items and activities) has been shown to be an undesirable condition. A responsible driverassistance smart phone application will minimize its complexity and interface in order not to create excessive risk (Young and Regan, 2007). Visual driver feedback in a dashboard context is most often correctly interpreted when presented in a 
toggle (on/off or yes/no) format, though the careful incorporation of incrementalformat information provides significantly more information without exposing the interface to too much risk of misinterpretation (Graving et al., 2010). Unfortunately, a second study of providing driving fuel-efficiency feedback found that detailed advice was required to significantly modify driving habits (van der Voort et al., 2001). Given the issues involved, the design of any driver-assistance smart phone application requires a thoughtful consideration of such human factors and a wellplanned implementation, in order to provide clear, accurate, contextual, and lowrisk information (van der Voort, 1997), perhaps using audio rather than visual cues.

\title{
2.3 Creating A Feedback Heuristic
}

\author{
"Essentially, all models are wrong, but some are useful." \\ GEORGE BOX AND NORMAN DRAPER, \\ EMPIRICAL MODEL-BUILDING AND RESPONSE \\ SURFACES, P. 424
}

Traffic models are mathematical descriptions and computer simulations that attempt to accurately describe the behavior of traffic based on a set of equations and rules. Accuracy, in this sense, is the level of agreement with empirical data sets of macroscopic traffic measurements. As such, being able to make predictions about traffic behavior which may later be observed is an important goal. Given their importance, traffic models have been and continue to be an area of active (and contentious) research. Reviews of the state of the art in traffic modeling can be 
found in Chowdhury et al. (2000), Helbing (2001), Maerivoet (2005); Maerivoet and De Moor (2005), Orosz et al. (2010), and Schadschneider et al. (2011).

Traffic models can be classified as either macroscopic or microscopic. Macroscopic models aim to predict the state of traffic as a whole over time via parameters such as flow, density, and speed. Microscopic models aim to mimic the behavior of individual vehicles in traffic and are considered valid if they produce macroscopic results that match empirical measurements (Maerivoet and De Moor, 2005). The remainder of this section will focus on microscopic traffic modeling approaches, given that the purpose of this work is to create a machine learning algorithm which can provide driver feedback at the individual level. Additionally, there is general agreement that only a microscopic model is capable of simulating flow breakdown, which is central to this work (Duncan and Littlejohn, 1997; Knospe et al., 2004; Treiber and Kesting, 2010).

There are three primary families of deterministic microscopic traffic models (Kesting et al., 2008a). The first family includes those models, such as Follow-theLeader and Optimal Velocity, which are continuous (that is, can be assigned any real number value) in all variables (time, distance, velocity, and acceleration). The second family comprises the many Traffic Cellular Automata models, including Nagel-Schreckenberg and its derivatives, which are discrete (that is, are restricted to a set of values) in all variables. The third family is a hybrid of the first two, discrete in time but continuous in the rest, based on the Coupled-Map Lattice. There are also a variety of other approaches to modeling traffic, including stochastic models, for which references to comprehensive reviews can be found at the beginning of the section. Given the goal of the proposed research, to isolate the conditions that 
will maximize the effect of a change in the behavior of a vehicle, and the need to compare its results with empirical data, it was determined that a model from the first family was the most appropriate (Treiber et al., 2000).

\subsubsection{Intelligent Driver Model}

The model selected to generate simulated data to train the heuristic at the heart of this thesis is a recent microscopic model which has withstood scrutiny in the literature, the Intelligent Driver Model (IDM) (Treiber et al., 2000, 2006b; Kesting et al., 2008a; Kesting and Treiber, 2008). It is one member of the Follow-the-Leader, Car-Following class of models with the following advantages:

1. It behaves as if accident-free because of the dependence on the relative velocity;

2. For similar reasons and because of metastability, it shows the selforganized characteristic traffic constants demanded by Kerner and Konhaüser, hysteresis [delay] effects, and complex [traffic] states;

3. All model parameters have a reasonable interpretation, are known to be relevant, are empirically measurable, and have the expected order of magnitude;

4. The fundamental diagram and the stability properties of the model can be easily (and separately) calibrated to empirical data;

5. It allows for a fast numerical simulation; and

An equivalent macroscopic version of the model is known, which is not the case for most other microscopic traffic models (Treiber et al., 2000, p. 1806).

Brockfeld et al. $(2003,2004,2005)$ examine the calibration and validation of a variety of microscopic models and found the Intelligent Driver Model to be as accurate as other, more complicated models (those with a larger number of parameters). It must be noted, however, that all models had a residual error rate of at 
least $15.5 \%$ compared to the empirical data used. In general, the simplest model is recommended, as more complex models were not found to be qualitatively better (Brockfeld et al., 2004), but it has been observed that any model is "appropriate for describing the average dynamic behavior" of traffic (Brockfeld et al., 2005). Hoogendoorn and Hoogendoorn (2010) confirm the findings of Brockfeld et al. (2004) and show that the IDM is statistically better than the (admittedly simple) Gazis, Herman, and Rothery model (Gazis et al., 1961). An open-source reference implementation of the IDM exists at the web site www.traffic-simulation.de, maintained by Martin Treiber (Treiber and Kesting, 2010).

\section{Mathematical Description of the Intelligent Driver Model}

The IDM attempts to address shortcomings of prior car-following models, which suffered from a variety of undesirable or unrealistic features, including symmetric acceleration and deceleration, lack of equilibrium values, unrealistically large or small acceleration and deceleration values, or lack of macroscopic features seen in empirical data. The IDM improves upon prior models by using an equation of acceleration which is dominated by the vehicle's desired velocity when the intra-vehicle gap is large and by the same gap when that gap is small. In turn, the vehicle seeks a desired gap which is a combination of minimum gap at maximum (jam) density, minimum time headway (amount of time to cover the distance) to the leading vehicle, and a term which increases as the vehicle's speed increases. The parameters of each vehicle are continuous in time and space and based on a car-following equation of acceleration. For a full theoretical discussion of the IDM, including how it improves upon specific car-following models, please see Treiber 
Table 2.1: Model Parameters of the Intelligent Driver Model

\begin{tabular}{lcl}
\hline \multicolumn{3}{c}{$\begin{array}{c}\text { Model Parameters of the IDM } \\
\text { (Treiber and Helbing, 1999; Treiber et al., 2000) }\end{array}$} \\
\hline IDM Parameter & Symbol & Typical value \\
\hline Time & $t$ & $\mathbf{R} \geq \mathbf{0}$ \\
Vehicle index & $\alpha$ & $\mathbf{Z} \geq \mathbf{0}$ \\
Vehicle length & $l_{\alpha}$ & $1 / \rho_{\max } \approx 5 m$ \\
Vehicle position & $x_{\alpha}(t)$ & $\mathbf{R} \geq \mathbf{0}$ \\
Vehicle velocity & $v_{\alpha}(t)$ & $\mathbf{R} \geq \mathbf{0}$ \\
Velocity difference & $\Delta v_{\alpha}(t)$ & $\mathbf{R}$ \\
Vehicle gap & $s_{\alpha}(t)$ & $\mathbf{R} \geq \mathbf{0}$ \\
Desired vehicle gap & $s_{\alpha}^{*}$ & $\mathbf{R} \geq \mathbf{0}$ \\
Desired velocity & $v_{0}$ & $120 \frac{\mathrm{km}}{\mathrm{h}}$ \\
Safe time headway & $T$ & $1.6 \mathrm{~s}$ \\
Acceleration & $\dot{v}_{\alpha}(t)$ & $\mathbf{R}$ \\
Maximum acceleration & $a$ & $0.73 \frac{\mathrm{m}}{\mathrm{s}^{2}}$ \\
Desired deceleration & $b$ & $1.67 \frac{\mathrm{m}}{\mathrm{s}^{2}}$ \\
Acceleration exponent & $\delta$ & $1-5$ \\
Jam distance & $s_{0}$ & $2 \mathrm{~m}$ \\
\hline
\end{tabular}

et al. (2000). In the interests of simplicity, the same notation used by Intelligent Driver Model articles in the literature will be used throughout this thesis, as detailed in Table 2.1.

Per the same sources, the equations defining the Intelligent Driver Model are as follows. The standard equation of change of position is:

$$
x_{\alpha}(t+\Delta t)=x_{\alpha}(t)+v_{\alpha}(t) \Delta t+\frac{1}{2} \dot{v}(t)(\Delta t)^{2}
$$

The standard equations of change of velocity are:

$$
v_{\alpha}(t+\Delta t)=v_{\alpha}(t)+a^{\alpha}(t) \Delta t
$$




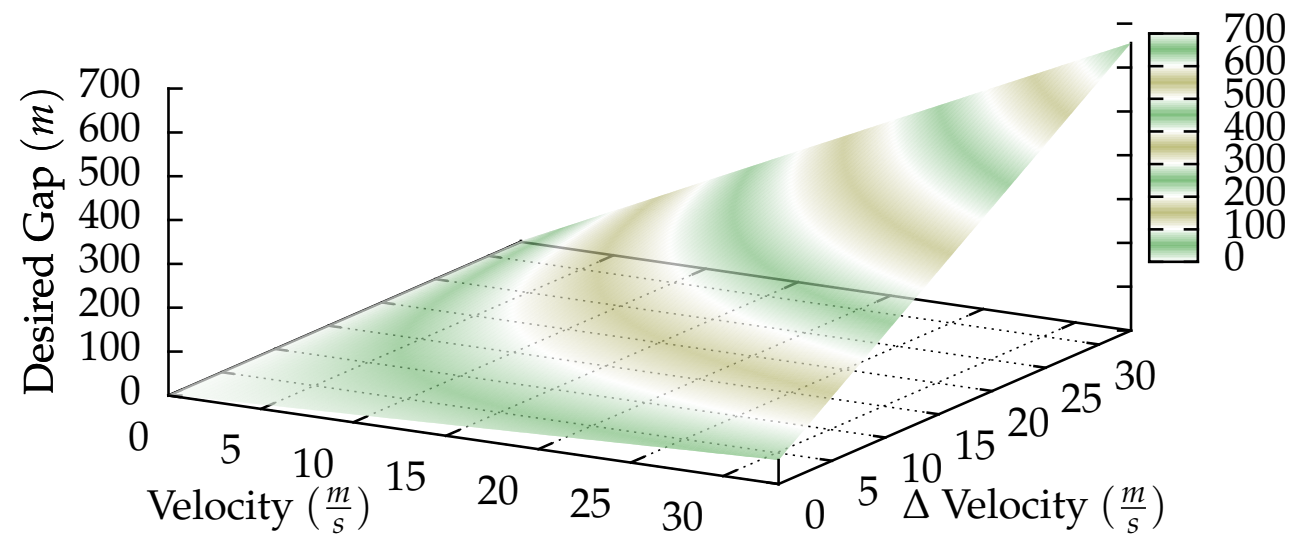

Figure 2.1: Desired gap as a function of the vehicle's velocity and the difference in velocity between the vehicle and its leading vehicle.

$$
\Delta v_{\alpha}(t)=v_{\alpha}(t)-v_{\alpha-1}(t)
$$

A prerequisite for the equation of acceleration is the desired gap $s^{*}$ :

$$
s^{*}\left(v_{\alpha}(t), \Delta v_{\alpha}(t)\right)=s_{0}^{\alpha}+v T^{\alpha}+\frac{v_{\alpha}(t) \Delta v_{\alpha}(t)}{2 \sqrt{a^{\alpha} b^{\alpha}}}
$$

Figure 2.1 shows the values for $s^{*}$ across the ranges that can be taken by $v$ and $\Delta v$ based on the desired velocity $v_{0}=120 \frac{\mathrm{km}}{\mathrm{h}}=33 . \overline{3} \frac{\mathrm{m}}{\mathrm{s}}$.

Finally, the equation of acceleration (denoted by $\dot{v}$ ) is:

$$
\dot{v}_{\alpha}(t)=a^{\alpha}\left[1-\left(\frac{v_{\alpha}(t)}{v_{0}^{\alpha}}\right)^{\delta}-\left(\frac{s^{*}\left(v_{\alpha}(t), \Delta v_{\alpha}(t)\right)}{s_{\alpha}(t)}\right)^{2}\right]
$$

Figure 2.2 shows the values for $\dot{v}$ across the ranges that can be taken by $v$ and $\Delta v$ based on the desired velocity $v_{0}=120 \frac{\mathrm{km}}{\mathrm{h}}=33 . \overline{3} \frac{\mathrm{m}}{\mathrm{s}}$ and an actual gap $s_{\alpha}=$ 


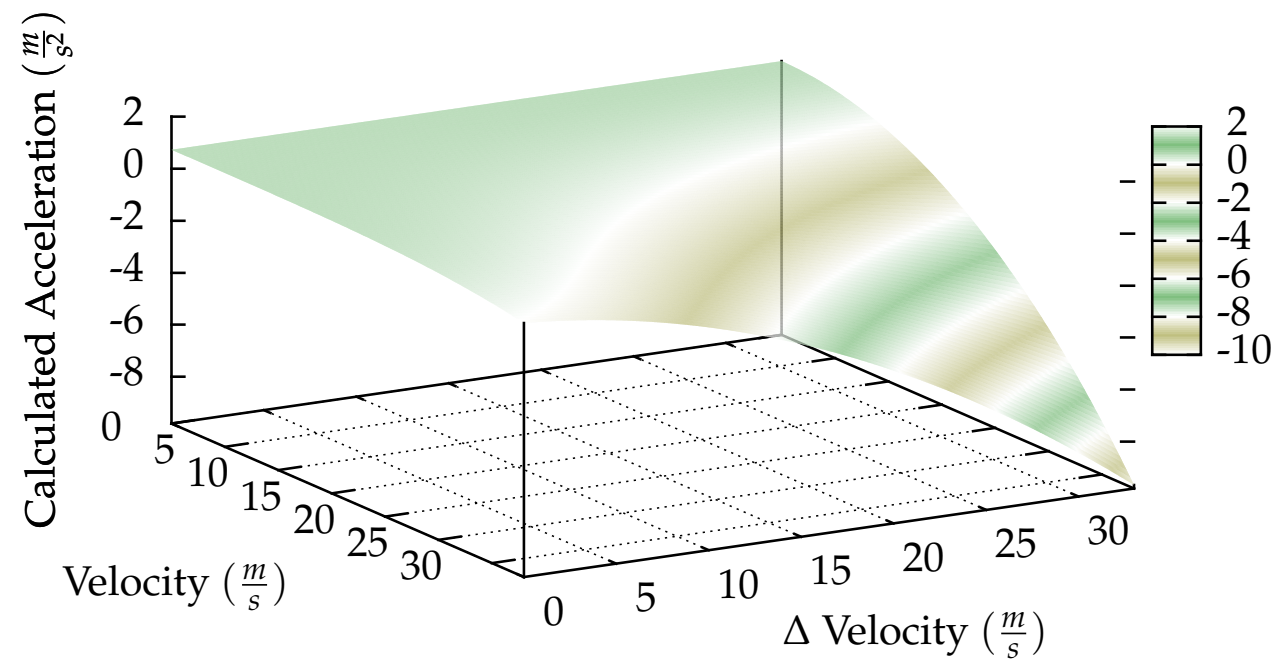

Figure 2.2: Acceleration as a function of the vehicle's velocity and the differences in velocity and actual gap between the vehicle and its leading vehicle.

$180 \mathrm{~m}$. Note that deceleration can exceed the desired deceleration $b$ if the vehicle is suddenly presented with unrealistic (dangerous) differences in velocity.

\subsubsection{Discrete Wavelet Transformations}

Wavelet transforms have become a major tool in the sciences since their introduction in 1982 (Sifuzzaman et al., 2009; Zheng et al., 2011). Wavelet transforms have a variety of applications in transportation, including incident detection, work zone traffic management, and flow forecasting (Adeli and Karim, 2005; Adeli and Jiang, 2009). See Jensen and Cour-Harbo (2001) for a brief introduction to the mathematics and a longer discussion of the implementation of wavelet transforms. In addition to their straightforward computation, wavelet transforms have the advantage over 
the Fourier transforms of being able to store local information about a signal, useful in identifying isolated features. In the context of this work, wavelet transforms:

- Can extract features (information) from a signal, in this case accelerometer readings;

- Can analyze noisy vehicle trajectory data, making microscopic analysis possible (Zheng et al., 2011);

- Can detect acceleration changes which can be fed to an external heuristic to recognize congestion and oscillation waves;

- Operate on a time resolution suitable for both effective detection of traffic conditions and deployment on a smart phone.

\subsubsection{Machine Learning Algorithms}

This work attempts to provide intelligent feedback to the driver or vehicle control system based on the current state of the vehicle's acceleration curve. The intelligent feedback must be based on some prior knowledge of traffic behavior and outcomes. It therefore requires a computational approach which will allow it to predict a favorable result in the future if it provides feedback to the driver based on what is currently known. It is impossible, of course, to know all possible traffic states, and so a generalization of conditions and outcomes is sought.

Machine Learning (ML) algorithms are those designed to learn from past experiences in order to correctly react to new experiences or to correctly group new experiences (Mitchell, 1997). Machine learning algorithms commonly work by abstracting input data relationships in space (Cluster analyses, Support Vector Machines, etc.) or in networks (Artificial Neural Networks, Decision Trees/Forests, Bayesian Networks, etc.), broadly speaking, through mathematical and statistical 
processes of classification and regression (Steinwart and Christmann, 2008). All machine learning algorithms require some kind of feedback about their decisions. In Supervised Learning and related approaches, the algorithms are trained by being given some number of classified training data initially on which to base future decisions. In Unsupervised or Reinforcement Learning, the algorithm seeks to either structure the data or to improve the feedback it receives (reinforcement) about its decisions (Mitchell, 1997).

In this thesis, actual individual drivers will not be used to pilot/test the intelligent feedback mechanism that is developed. Rather, many different traffic scenarios will be identified through systematic simulation. The events obtained from the simulations (i.e. traffic conditions and driver response) will then be classified as positive or negative based on their effect on traffic flow. These simulations will then be used to train a supervised machine learning algorithm. Specifically, the Support Vector Machine technique is used as it is particularly well-suited to the task.

\section{Support Vector Machines}

A Support Vector Machine (SVM) is a type of classifying machine learning algorithm, that is, it attempts to correctly classify new inputs based on a set of training data whose classes are also known. This works by seeking a binary classification of the current state of the vehicle: provide no feedback, or provide feedback to the driver or vehicle control system to take action. Unary, binary, and multi-class classification is one of the main applications of Support Vector Machine techniques (Steinwart and Christmann, 2008). 


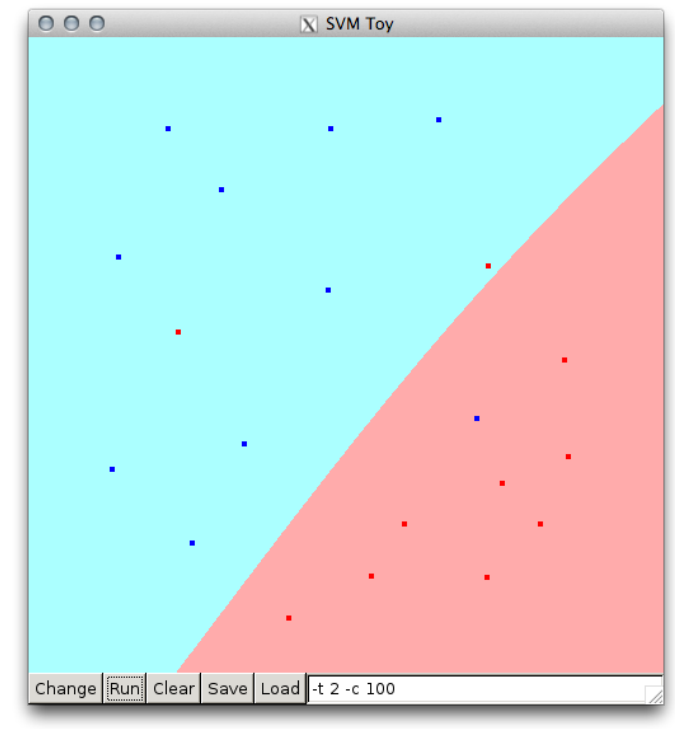

(a) Two classes

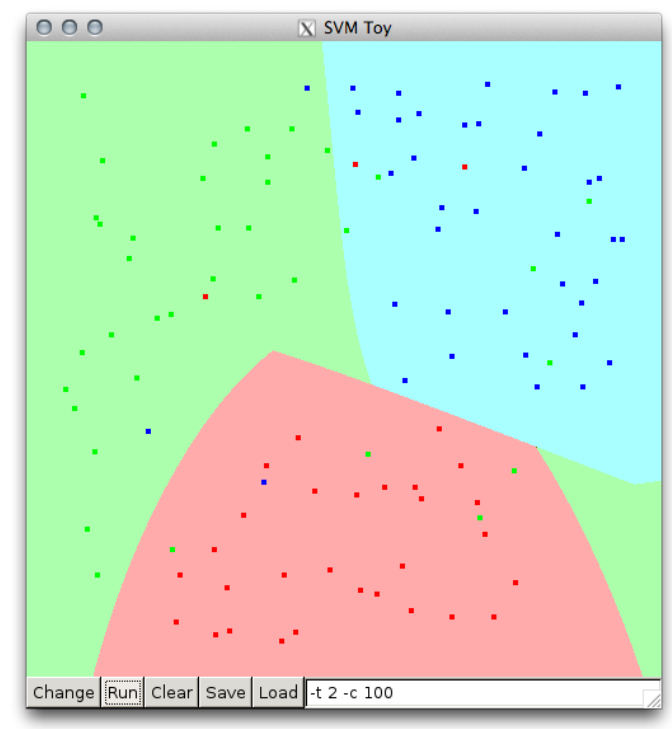

(b) Three classes

Figure 2.3: Example Support Vector Machines 
A Support Vector Machine functions by calculating a hyperplane (an n-dimensional plane) that spatially separates the two classes (in the case of binary classification) of training data inputs (Figure 2.3). The hyperplane is called the decision boundary, and the support vector machine attempts to maximize the width of the margin to either side of the boundary as a criterion of finding a near-optimal solution. Each input is a vector, the number of elements of which determines the lower bound of the dimensionality of the machine calculation. In a so-called hard margin SVM, the hyperplane must perfectly (with no error) separate the two classes. This, however, can lead to problems of solvability and overfitting, overfitting being the finding of a solution which is too specific to the training data set used, mainly by forcing the solution calculation into a high number of dimensions. The soft margin SVM allows for some degree of misclassification in order that the solution is not overfitted to the training data set. It becomes the task of the support vector machine to minimize misclassification while maximizing the width of the margin. According to Abe (2010), Support Vector Machines exhibit the following advantages and disadvantages compared to multilayer neural networks:

\section{Advantages}

1. Maximization of generalization ability. [.... Because] a support vector machine is trained to maximize the margin, the generalization ability does not deteriorate very much, even [when training data are scarce and linearly separable].

2. No local minima. [.... Because] a support vector machine is formulated as a quadratic programming problem, there is a global optimum solution.

3. Wide range of applications. [...] In support vector machines, generalization ability is controlled by changing a kernel, its parameter, and the margin parameter. [....] 
4. Robustness to outliers. [....] In support vector machines the margin parameter $\mathrm{C}$ controls the misclassification error. If a large value is set to C, misclassification is suppressed, and if a small value is set, training data that are away from the gathered data are allowed to be misclassified. Thus by properly setting a value to $C$, we can suppress outliers.

\section{Disadvantages}

1. Extension to multiclass problems. Unlike multilayer neural network classifiers, support vector machines use direct decision functions. Thus an extension to multiclass problems is not straightforward, and there are several formulations. [....]

2. Long training time. Because training of a support vector machine is done by solving the associated dual problem, the number of variables is equal to the number of training data. Thus for a large number of training data, solving the dual problem becomes difficult from both the memory size and the training time [...].

3. Selection of parameters. In a support vector machine, we need to select an appropriate kernel and its parameters, and then we need to set the value to the margin parameter $\mathrm{C}$. To select the optimal parameters to a given problem is called model selection. [....] In support vector machines, model selection is done by estimating the generalization ability through repeatedly training support vector machines. [....]

Abe, 2010, pp. 58-59.

This work avoids the first disadvantage entirely in that it seeks binary classification only. The second and third disadvantages can be overcome by the application of additional computation time, which has an ever-decreasing cost.

\subsubsection{Implications for the Present Work}

While elimination of congestion remains the ultimate goal, the problem can be addressed in multiple ways. Systemic approaches such as congestion pricing, 
ramp metering, and variable speed limits are not the focus of this work. Rather, a microscopic, driver-assistance approach will be discussed. The goal of this thesis is to describe a method to smooth, and ultimately harmonize, vehicle speed overall through individual vehicle perturbations. This will have the net effect of introducing destructive interference to the stop-and-go velocity wave, decreasing the amplitude of velocity oscillations in congested traffic, and leading to flow recovery.

In the physical sense, at the level of a participating vehicle, the vehicle will not accelerate quite as much as it may have in the absence of feedback, while not having to decelerate as much in turn. This creates a positive feedback loop for following vehicles and the velocity shockwave is not amplified, or, ideally, is dampened as it propagates upstream. Therefore, there is both a driver and societal benefit to be realized.

The proposed heuristic is devised as a machine learning algorithm trained to make constructive recommendations to the driver based on acceleration data from the smart phone's accelerometer while in urban freeway traffic. The motivation behind the development of a smart phone application is to be able to advise the individual driver when to cease acceleration in anticipation of the next braking action in the cycle. In this way, the driver will inject information into the system that will dampen oscillations. In effect, this attempts voluntary, single-driver Intelligent Speed Adaptation, a method a managing vehicle speeds on roadways (Servin et al., 2006). The intelligent feedback application will appeal to operational and strategic behaviors of the driver-agent model (Kesting et al., 2008a) in (Uhrmacher and Weyns, 2009): limited estimation capability, limited attention span, desired acceleration and deceleration, smooth driving, and so on. Drivers are also thought to 
be most receptive to modifying behaviors when fuel savings can be shown (Gonder et al., 2012). Intelligent feedback will help drivers refrain from rapid acceleration and hard braking at one extreme to moderate acceleration ( $3 \mathrm{mphs}$ Gonder et al., 2012) and light braking to eliminating acceleration and deceleration completely (Gonder et al., 2012). Addressing these problems will hopefully minimize the kinetic intensity of the commute and reduce environmental impact. Deploying the heuristic in a smart phone application would therefore meet the requirements set out in van der Voort (1997, p. 73):

It has been concluded that for a device to significantly improve fuel economy, it should:

- provide the driver with clear, accurate, and non-contradictory information

- take into account the context a vehicle is in

- place no requirements on the driver which are too high to combine with the actual driving task

- work within an urban environment.

An additional requirement is that the device needs to be cost-effective.

Gonder et al. (2012) describe how "speed/acceleration data [...] from the GPS/accelerometer on a non-connected smart phone can provide the feedback basis for the most critical fuel efficiency behaviors" (p. 459). Van der Voort (1997) also recommend the accelerometer approach for its ability to detect and measure hard braking, in itself undesirable driving behavior and indicative of prior acceleration to too high a speed. A smart phone application incorporating the proposed intelligent feedback would have to alert the driver appropriately and display the effect of the driver's action, based on whether the driver acted on the feedback. 
In lieu of experimenting on actual drivers, the proposed machine learning algorithm will be trained and evaluated based upon simulations of individual vehicles using a microscopic traffic model. This model will collect acceleration data from a sensor vehicle, change the acceleration behavior of the sensor vehicle, and determine, both through calculation of kinetic intensity and macroscopic measures of the simulated traffic, whether its recommendations improve the commute for the sensor vehicle individually and the system as a whole, respectively. 


\section{Chapter 3}

\section{Methods}

This section of the thesis details the various processes used to implement an instance of the Intelligent Driver Model (Treiber et al., 2000; Kesting et al., 2008a). This simulation routine is implemented for high performance computing and capable of assessing the behavior of individual vehicles. Also, the wavelet transforms used to refine the simulation data and ultimately provide information to the support vector machine will be described.

\subsection{Implementation Of The Intelligent Driver Model}

It is desirable to generate machine learning algorithm training data that covers a wide variety of traffic conditions, but simulating these conditions may require a significant amount of time and computing resources. The software tools used to simulate traffic and generate test events were chosen with an eye toward High Performance Computing. Specifically, the equations at the center of the simulator, 
which come from the works of Treiber, Hennecke, Helbing, and Kesting (Treiber et al., 2000; Kesting et al., 2008a) on the Intelligent Driver Model (Section 2.3.1), are implemented in the $\mathrm{C}$ programming language with OpenMP compiler directives for parallel processing. The GNU C Compiler gcc version 4.8.2 (Free Software Foundation, Inc., 2014) is used, which supports the OpenMP version 3.1 specification (Free Software Foundation, Inc., 2013). The choice of a parallel programming architecture to simulate vehicle movement and generate test events is solely to increase the speed at which it executes. In fact, the OpenMP compiler directives can be disabled at compile-time, resulting in a correct, serial-processing program.

\subsubsection{Simulator Architecture}

The architecture of the traffic simulator is as follows. The primary transportation infrastructure is of a one-lane road segment of a user-specified length, containing information about the road segment itself (designation, lane number, length, upstream road segment reference, downstream road segment reference, and detector references), virtual detectors collecting macroscopic traffic characteristics (density, flow, and velocity) and an array of data structures containing information about vehicles on the road segment (current position, velocity, acceleration, and kinetic intensity). As this simulation is designed to run in a high-performance-computing parallel-processing environment, the array is statically allocated in memory, rather than implemented using object-oriented programming, as in the source code available from Treiber (2011) (Section 2.3.1). The array is sized to hold information about the number of vehicles that could be present at jam density on the specified length 
of road, plus a small margin of error in case the road segment is pushed beyond jam density conditions.

As the array is statically allocated, information about the vehicles does not change position within the array as might be expected in a dynamic-list data structure. Instead, when a vehicle is added as the upstream-most vehicle, it remains at the assigned array index until it becomes the downstream-most vehicle, moves beyond the length of the road segment during a single time step, and is moved to the downstream road segment. Possible initial conditions are illustrated pictographically:

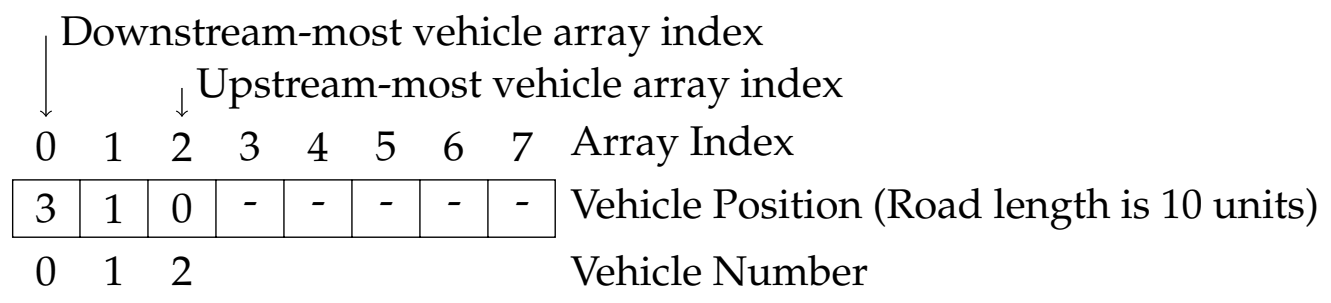

Intermediate steps are skipped as vehicles advance along the road segment uniformly. The lead (downstream-most) vehicle eventually reaches the end of the road segment:

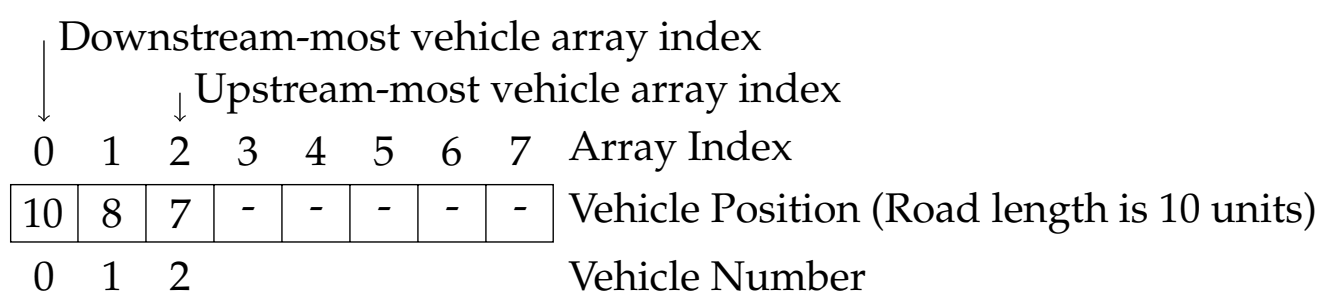

The lead vehicle now moves to a downstream road segment and is removed from the current road segment: 


\begin{tabular}{|c|c|c|c|c|c|c|c|c|}
\hline \multirow[b]{2}{*}{0} & \multicolumn{8}{|c|}{$\begin{array}{l}\text { Downstream-most vehicle array index } \\
\downarrow \text { Upstream-most vehicle array index }\end{array}$} \\
\hline & 1 & 2 & 3 & 4 & 5 & 6 & 7 & Array Index \\
\hline- & 9 & 8 & - & - & - & - & - & Vehicle Position (Road length is 10 units) \\
\hline
\end{tabular}

A new vehicle might enter the road segment at its beginning and is added at the next available higher array index:

\begin{tabular}{|c|c|c|c|c|c|c|c|c|}
\hline \multirow[b]{2}{*}{0} & \multicolumn{8}{|c|}{$\mid \begin{array}{c}\text { Downstream-most vehicle array index } \\
\downarrow \text { Upstream-most vehicle array index }\end{array}$} \\
\hline & 1 & 2 & 3 & 4 & 5 & 6 & 7 & Array Index \\
\hline- & 9 & 8 & 0 & - & - & - & - & Vehicle Position (Road length is 10 units) \\
\hline
\end{tabular}

New vehicles enter the ring road in two ways. The first is through an approximation of a ramp merge. The second is by the vehicle passing from the end of the upstream road segment to the beginning of the current road segment. In the case of the ring road implemented in this thesis, this means that a vehicle moves off the end of the road to the beginning of the same road; the ring road is its own upstream and downstream. Each time a vehicle enters the road by either method, it is assigned a new number. Vehicle numbers only increment as implemented in this simulator. Eventually, the downstream-most and upstream-most vehicle numbers will be greater than the length of the array, as vehicles are added to the end and removed from the beginning. As such, modulo arithmetic with the array length as the divisor is used to keep information about vehicles within the array bounds. It is therefore inevitable to have vehicles in a road segment arranged as follows: 


\begin{tabular}{|c|c|c|c|c|c|c|c|c|}
\hline \multirow[b]{2}{*}{0} & & \multicolumn{7}{|c|}{ Upstream-most vehicle array index } \\
\hline & 1 & 2 & 3 & 4 & 5 & 6 & 7 & Array Index \\
\hline 3 & 2 & - & - & - & 6 & 5 & 4 & Vehicle Position (Road length is 10 units) \\
\hline
\end{tabular}

This static memory architecture allows the system to maximize CPU cycles spent on simulation computation, rather than memory management, as would be the case in a dynamic memory allocation architecture.

In light of the parallel programming environment employed here, it is important to note that two sets of the physical characteristics (acceleration, velocity, position, and kinetic intensity) of vehicles are stored in the vehicle data structure. This is so that present characteristics of a vehicle may be read for the calculations of another (i.e. following) vehicle's future characteristics even while new characteristics for the vehicle are being written, eliminating a significant data dependency from the simulation. Read-and-write behavior is alternated by a binary modulo operation against the (integer) simulation clock (Table 3.1). While this approach doubles the memory requirements for each vehicle, those additional memory requirements are on the same scale as the memory requirements that would be necessary for implementing linked list or object reference pointers in a dynamic memory allocation architecture, so the overall penalty is small.

Finally, while the simulation leverages the OpenMP library for parallel processing, OpenMP provides only pseudo-parallel processing by means of process threads, rather than true Single-Instruction-Multiple-Data (SIMD) parallel processing provided by true high performance computing systems or General Purpose 
Table 3.1: Controlling read and write operations to memory in a parallel programming environment.

\begin{tabular}{rc|cc|cc|c}
\hline Clock & 0 & & 1 & \multicolumn{2}{c}{2} \\
\hline Pass & 0 & \multicolumn{2}{c}{1} & \multicolumn{2}{c}{0} \\
\hline Parameter Set & 0 & 1 & 0 & 1 & 0 & 1 \\
Allowed Action & Read & Write & Write & Read & Read & Write \\
Position & 0 & 1 & 2 & 1 & 2 & 3 \\
Velocity & 6 & 7 & 7 & 7 & 7 & 6 \\
Acceleration & 0 & 1 & -1 & 1 & -1 & 0 \\
Kinetic Intensity & 1 & 2 & 2 & 2 & 2 & 3 \\
\hline
\end{tabular}

Graphics Processing Units (GPGPUs). This work primarily leverages OpenMP's capacity for loop unrolling, the ability to process multiple loop iterations in parallel.

\subsection{Simulation}

This experiment simulates single-lane ring roads of varying lengths beginning at 19 vehicles per kilometer and increasing to approximately 62 vehicles per kilometer, at which threshold the simulation terminates (Figure 3.1). All vehicles are modeled to be homogeneous, sharing the same physical characteristics and behaviors. The fundamental activity of the simulator at each time step is to calculate the Intelligent Driver Model equations of the desired gap, acceleration, velocity, and position for each vehicle on the road and update them accordingly. Again, the purpose of the simulations is to generate data on traffic conditions and driver response that will become a corpus for training the machine learning algorithm. The flowchart in Figure 3.1 shows the general computational structure of the simulator that implements the Intelligent Driver Model. The sole purpose of the simulator is 


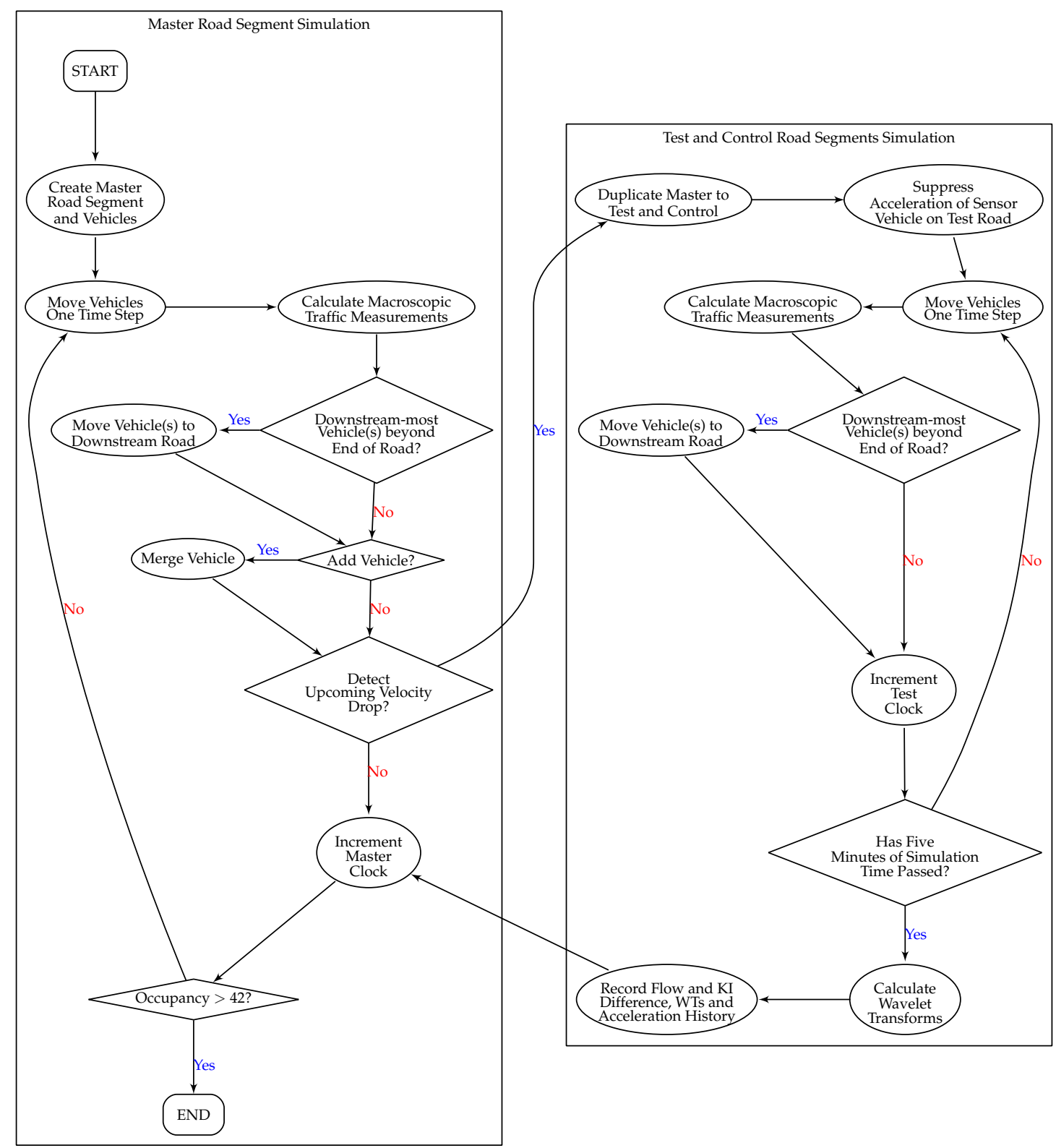

Figure 3.1: High-level Flowchart of the Proposed IDM Simulation Project. 

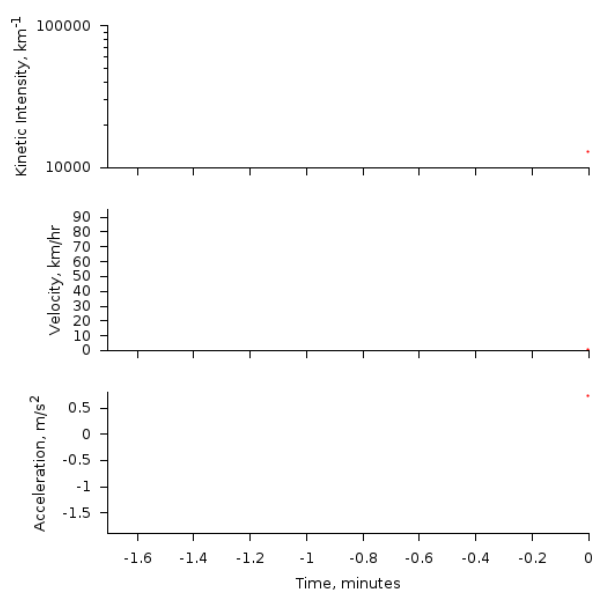

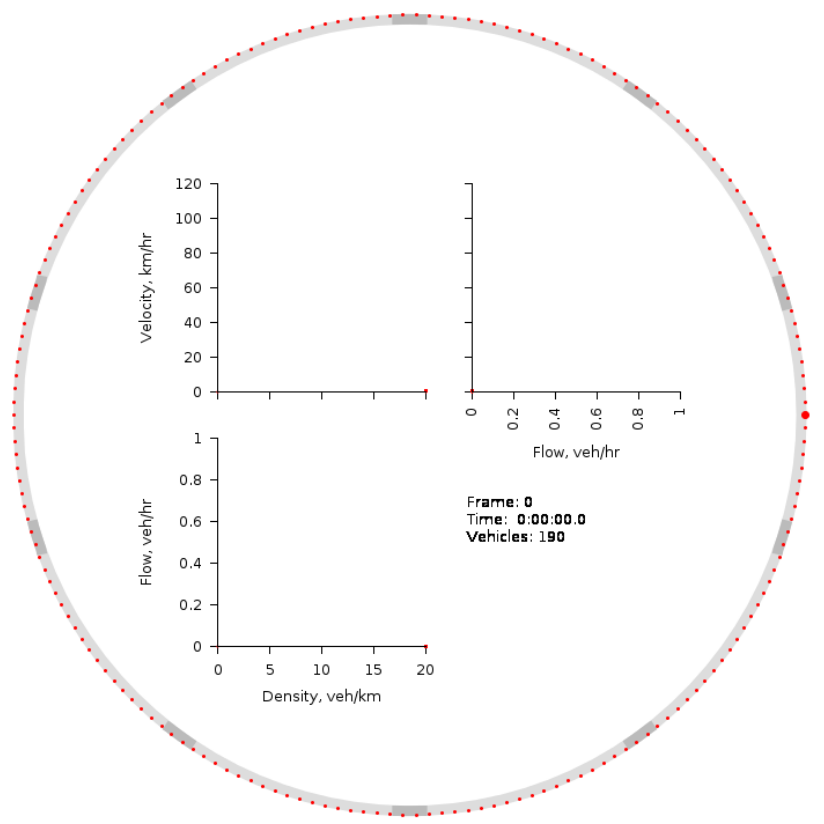

Figure 3.2: Display of the IDM simulator at clock step 0 for a $10 \mathrm{~km}$ ring road. The light gray circle is the road; the medium gray patches represent the sensing area of macroscopic traffic detectors. The red dots represent the location of vehicles on the road. The large dot is the sensor vehicle. Fundamental macroscopic diagrams are inside the road; the acceleration and velocity of the sensor vehicle and kinetic intensity for all vehicles on the road appear in the graphs to the left of the road. 
Table 3.2: Parameters of the IDM Simulator

\begin{tabular}{ll}
\hline \multicolumn{2}{c}{ Parameters of the IDM Simulator } \\
\hline IDM Parameter & Typical value \\
\hline Vehicle length & $5 \mathrm{~m}$ \\
Desired velocity & $120 \frac{\mathrm{km}}{\mathrm{h}}=33 . \overline{3} \frac{\mathrm{m}}{\mathrm{s}}$ \\
Safe time headway & $1.6 \mathrm{~s}$ \\
Maximum acceleration & $0.73 \frac{\mathrm{m}}{\mathrm{s}^{2}}$ \\
Desired deceleration & $1.67 \frac{\mathrm{m}}{\mathrm{s}^{2}}$ \\
Acceleration exponent & 4 \\
Jam distance & $2 \mathrm{~m}$ \\
Time step & $0.2 \mathrm{~s}$ \\
Merge interval & $10 \mathrm{~s}$ \\
Velocity drop threshold & $24 \frac{\mathrm{km}}{\mathrm{h}}$ \\
Sensor vehicle data history length & $102.4 \mathrm{~s}$ (512 time steps) \\
\hline
\end{tabular}

to operate vehicles on a roadway, look for the future occurrence of a velocity drop by the sensor vehicle, then conduct an experiment which compares traffic measurements between two different scenarios to generate a test event.

\subsubsection{Virtual Macroscopic Traffic Detectors}

The simulator creates one virtual macroscopic traffic detector collecting density, flow, and velocity values for each kilometer of road length. The simulator updates these macroscopic traffic parameters at each time increment over the simulated period based on the location and speed of all vehicles (the fleet) on the road. The simulator is configured to calculate density at each sensor based on the number of vehicles within 50 meters to either side of the detector; calculates flow based on the number of vehicles that have passed the detector in the previous 30 seconds; and 
calculates velocity based on the average space-mean-speed of the vehicles within 50 meters to either side of the detector. However, the simulator could be easily modified to accommodate any other parameter settings. Visual representations of the state of the road use all three parameters to create the fundamental diagrams of macroscopic traffic models, while the simulator uses only flow as part of the output data.

\subsubsection{Vehicle Merging}

The simulator is designed to attempt to merge an additional vehicle onto the road at position 0 (zero) at a user-defined interval (ten seconds in this application) of simulation time using a deterministic, ramp-meter approach. If there is sufficient gap between the vehicles immediately downstream and upstream of position 0 , a new vehicle is added at the average of the down- and upstream vehicles' position, with the velocity and acceleration of the downstream vehicle. While artificial, it approximates realistic merging behavior by bisecting the available gap and matching the speed and acceleration of the leading vehicle. If there is an insufficient gap between the vehicles immediately downstream and upstream of position 0 , the simulator will continue to attempt to add a vehicle each subsequent time step until it succeeds.

\subsubsection{Sensor Vehicle}

At the beginning of each run, the simulator designates one active vehicle as the sensor vehicle, recording its acceleration and velocity at each time step of the 
simulation. Additionally, the simulator calculates, sums, and records the kinetic intensity (Section 2.1.3) of the fleet with the sensor vehicle data. In all other respects, the sensor vehicle is identical to all other vehicles on the road.

\subsubsection{Master Road Simulation}

As the nature of this work is to address stop-and-go traffic by recognizing patterns in the acceleration curve of a participating vehicle, we are interested in future velocity drops by the sensor vehicle. At 0.2 second intervals throughout the simulation, an ancillary 102.4-second look-ahead simulation is conducted to help detect any upcoming significant decline (i.e. below $20 \%$ of the desired velocity) in the sensor vehicle's velocity. Therefore, at each time step of the simulation, the simulator replicates the existing road and conditions-its detectors, its vehicles, and their state- to conduct a look-ahead. The simulator then runs this ancillary system forward in time, up to the duration of the sensor vehicle's data recorder (512 time steps or 102.4 seconds at 0.2 seconds per time step as implemented in this experiment). If the simulator does not detect a velocity drop by the sensor vehicle in the look-ahead system, then the original road simulation resumes and the look-ahead system is discarded. If a velocity drop is predicted, the master simulation is paused and duplicated to create test and control simulations to assess the ramifications of suppressed acceleration (test) and unmodified behavior (control) responses. 

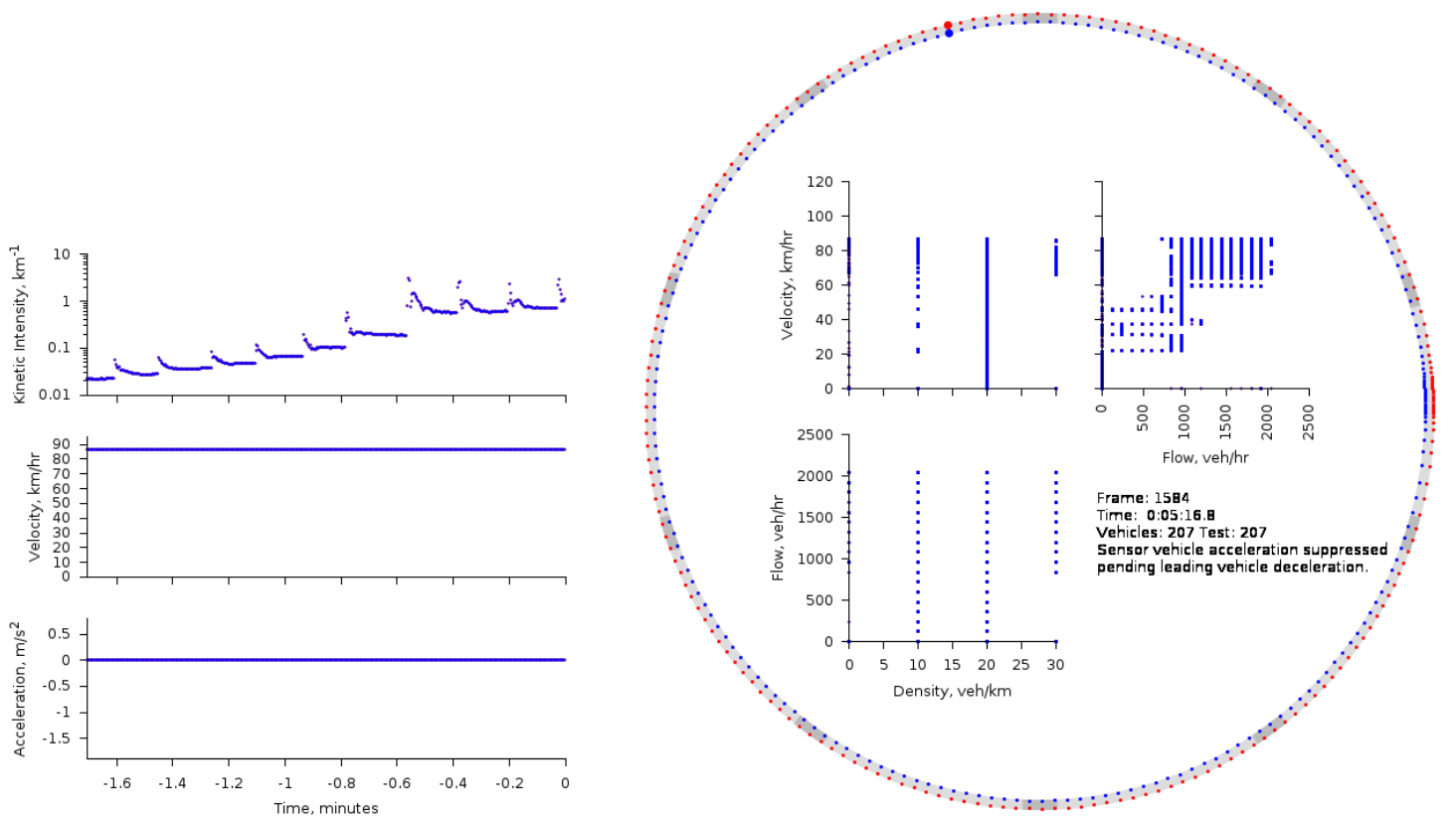

Figure 3.3: Display of the IDM simulator at the beginning of a test run. The blue and red vehicles are the same vehicles on the same road, not a two-lane road. The test sensor vehicle (large blue dot) has been instructed to maintain velocity. 

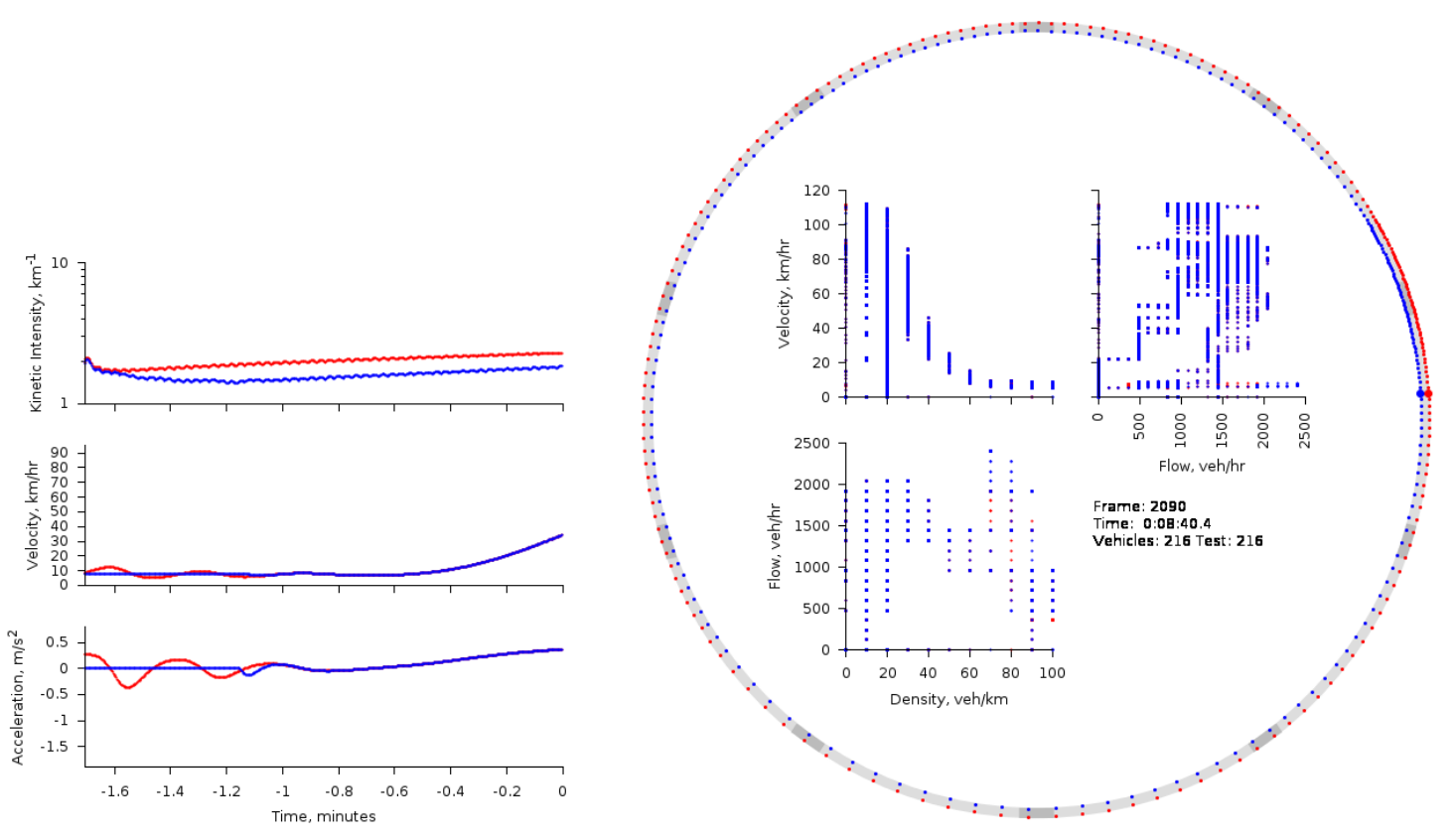

Figure 3.4: Note how the control sensor vehicle has accelerated and decelerated repeatedly, while the test sensor vehicle has been able to maintain its speed for over a minute, braking only slightly before exiting the congestion. 

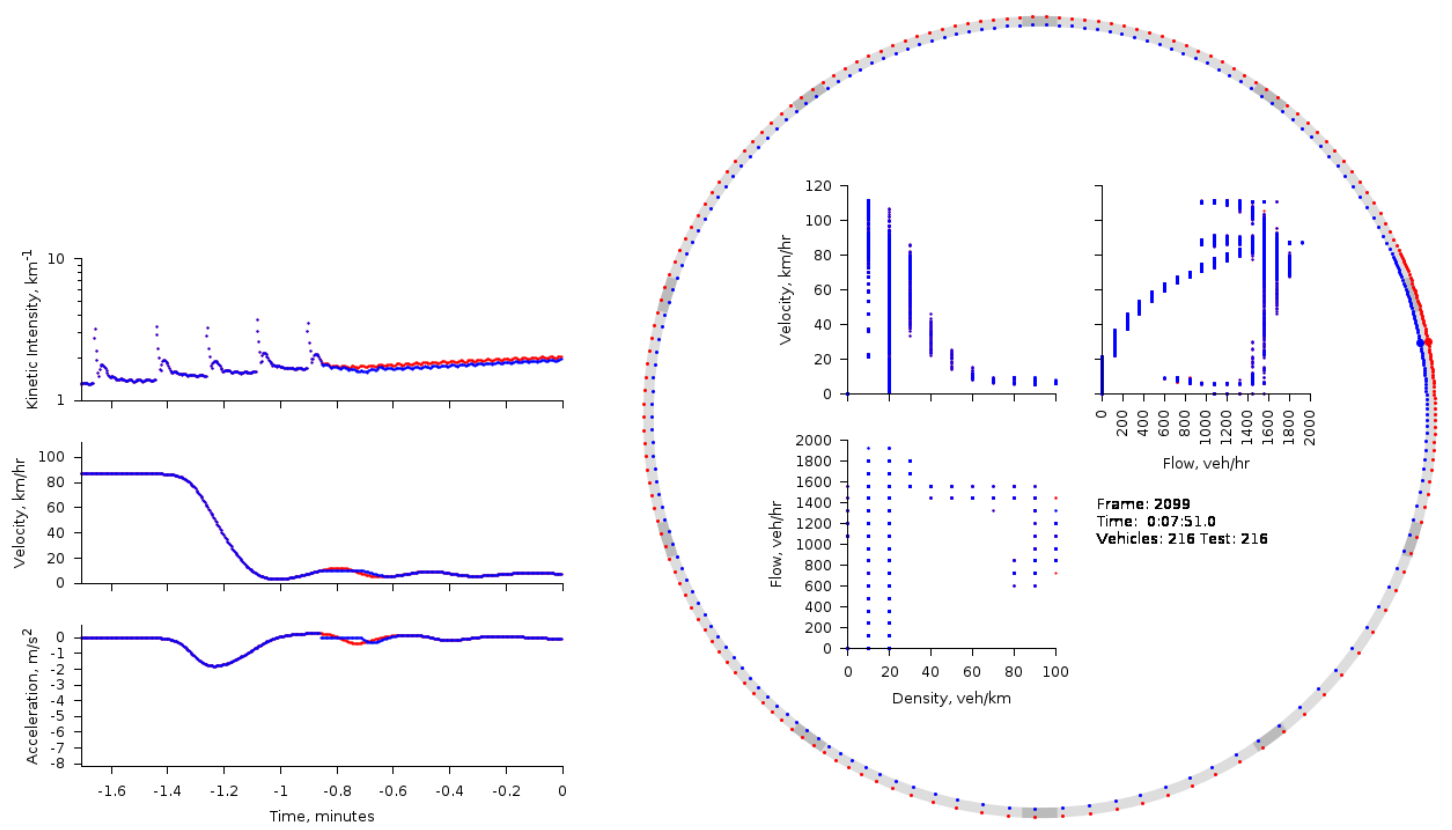

Figure 3.5: The short, 8.8-second delay in acceleration in this figure resulted in a $2.4 \%$ improvement in flow on the test road compared to the control road.
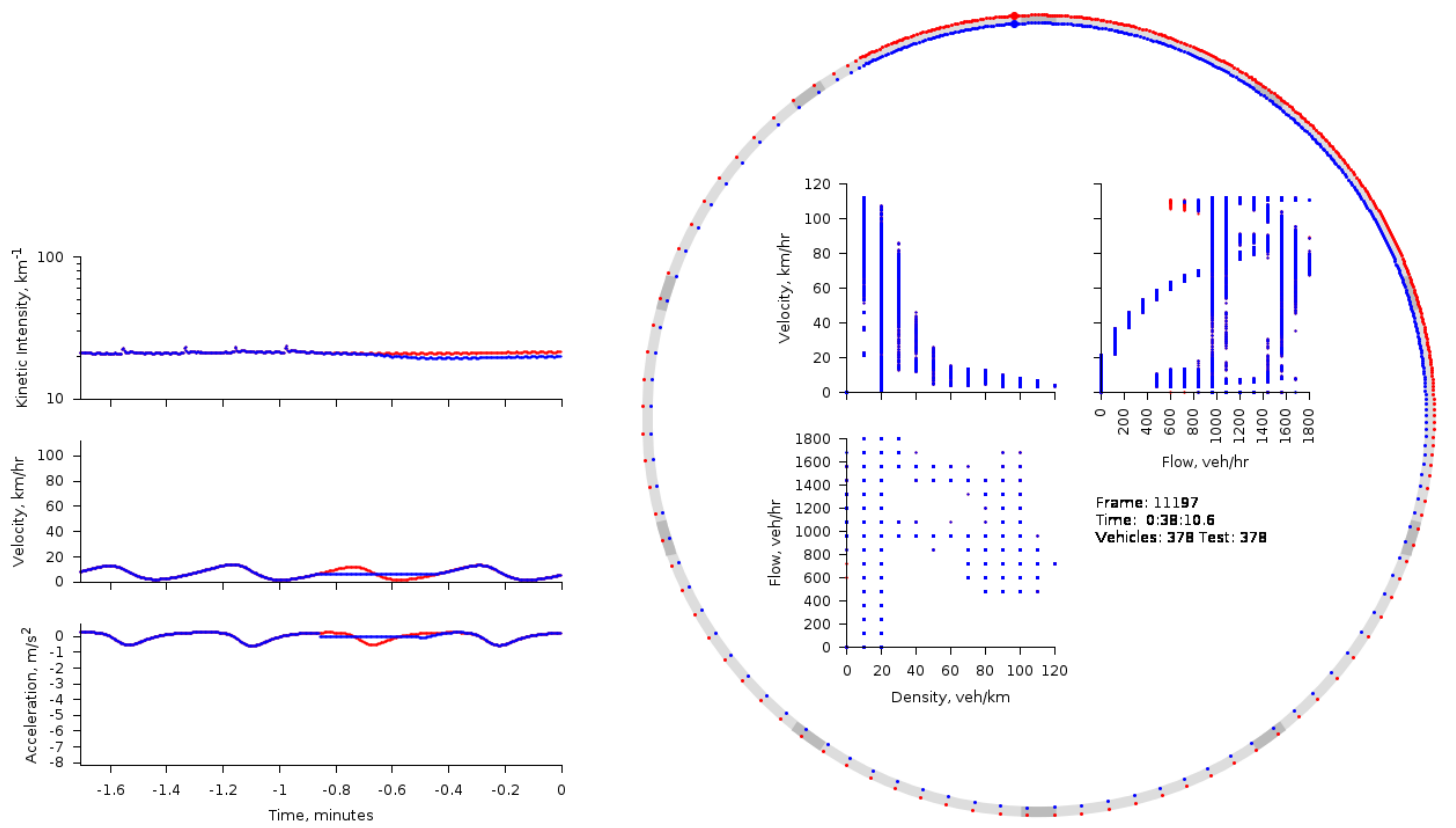

Figure 3.6: A 20.8-second delay in acceleration in this figure resulted in a $2.3 \%$ improvement in flow on the test road compared to the control road. 

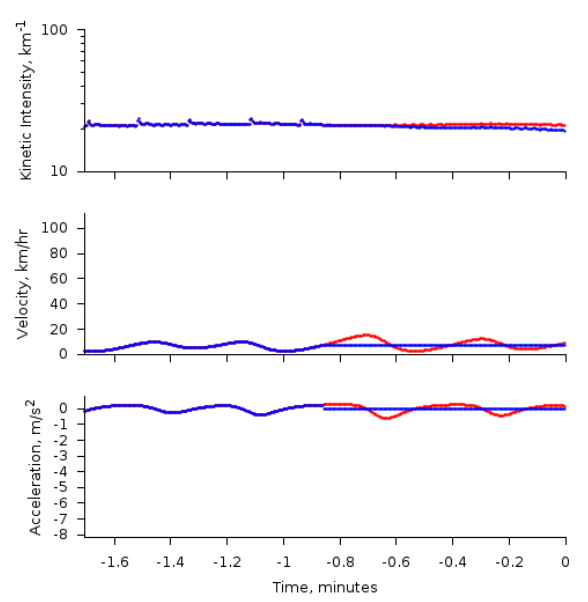

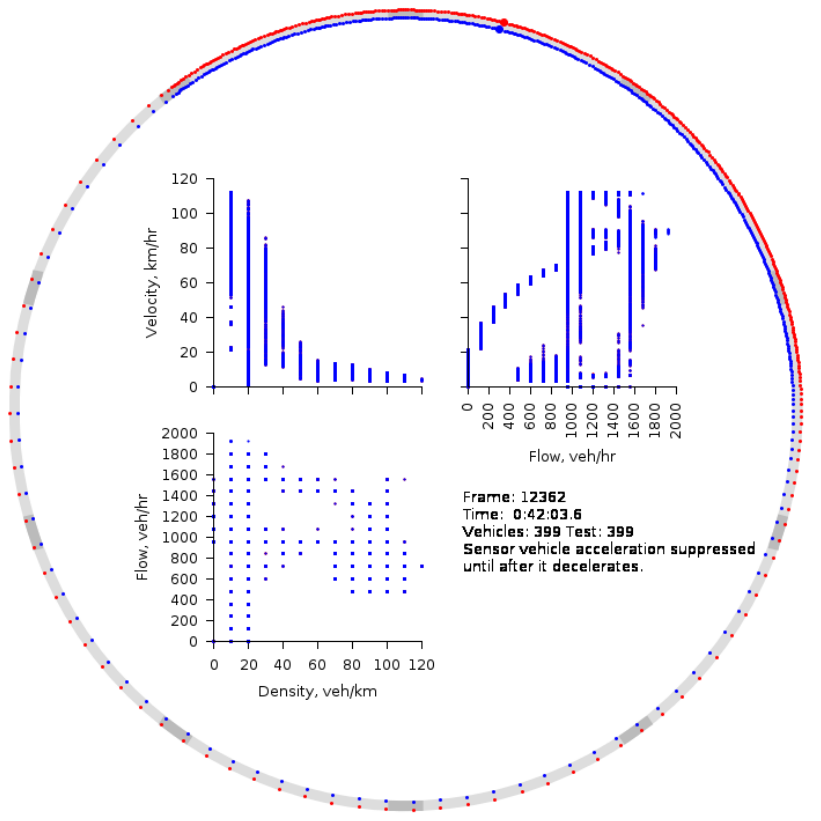

Figure 3.7: In breakdown conditions, acceleration suppression can go on for a very long time, 105.4 seconds in this test, resulting in a $2.1 \%$ improvement in flow.

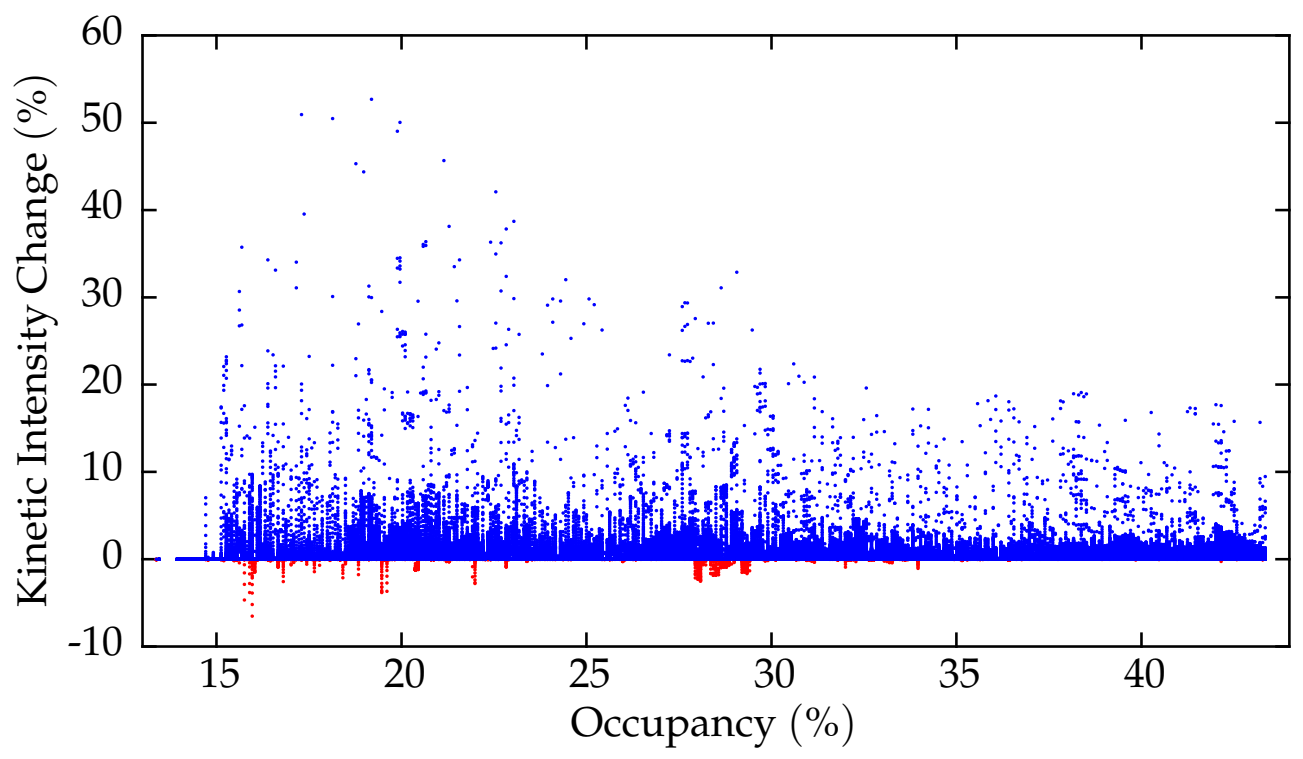

Figure 3.8: Plot of Percent Road Occupancy versus Kinetic Intensity. 


\subsubsection{Testing Modified Behavior of the Sensor Vehicle}

When the simulator detects an upcoming velocity drop, it creates two copies of the simulated system at that point in time, including its detectors, its vehicles, and their state: one copy is used as a test and the other as a control (Figure 3.3). A flag in the sensor vehicle on the test system which prohibits the sensor vehicle from accelerating until the IDM equation of acceleration becomes negative, that is, the sensor vehicle is required to maintain its velocity until it must brake. This is the sole change in state and behavior between the two, otherwise identical, roads and fleets.

An explanation of the chosen behavior modification is appropriate at this point. Assume that two vehicles in an urban highway setting are interacting; that is, the accelerating and braking (longitudinal) actions of the leading vehicle have an immediate impact on the following vehicle, inducing it to accelerate or requiring it to brake, respectively. If the following vehicle was to detect that the leading vehicle was accelerating with respect to the following vehicle (the gap between the vehicles is increasing), the following vehicle would normally be induced to accelerate in order to reach its desired velocity. If, however, the following vehicle chose or was advised not to accelerate, the gap between the vehicles would continue to grow until both vehicles reached the same velocity at some later time. Conversely, if the following vehicle was to detect that the leading vehicle was braking with respect to the following vehicle (the gap between the vehicles is decreasing), the following vehicle would normally be required to brake in order to avoid a collision. If, however, the following vehicle chose or was advised not to brake, the gap between the vehicles would continue to shrink until the vehicles collided. It is the opinion of this author that it is unsafe to pursue a heuristic that might tell a driver 
not to brake. The modified behavior of the sensor vehicle will be limited to not accelerating when it might otherwise be induced to accelerate.

After the test and control roads have been created and the behavior of the sensor vehicle has been modified, the simulator then runs the test and control systems forward in time (without adding new vehicles) for five minutes, calculating microscopic (kinetic intensity) and macroscopic (flow) traffic parameters as always. At the end of five minutes of simulated time, the simulator calculates the differences in total kinetic intensity and flow between the test and control systems. The simulator records these data, along with a wavelet transform of the acceleration data from the sensor vehicle leading up to the test. In this way, the simulator is able to record the effect for the entire fleet, positive or negative, of the sensor vehicle taking a particular action (maintaining velocity until obliged to brake), given an initial condition-the shape of the acceleration curve-prior to taking the action.

Another unknown in the search for accurate driver feedback is with regard to how much acceleration data history leading up to a suppressed acceleration action is required to generate an accurate prediction. Therefore, the simulator records multiple versions of each test event, so that later steps may compare feedback prediction accuracy and determine the best acceleration-data history length.

Each event from the test/control simulations produces two inputs for the training the support vector machine. The first input is a wavelet transform of the acceleration data leading up to the beginning of each test run. The second input is the result from the test simulation, how much flow was increased or decreased by the changed behavior of the sensor vehicle on the test system compared to the control system. The amount by which kinetic intensity increased or decreased is 
also produced by the simulator as an input (Figure 3.8), but with vanishing few exceptions (shown in red below $y=0.0$ ) it is always less (shown in blue above $y=0.0$ ) on the test road than the control road, and is not used in the analysis.

\subsection{Wavelet Transforms}

Raw acceleration data are undesirable for sending to a machine learning algorithm. Certainly, in a real-world situation, accelerometer data will contain some degree of noise: vehicle vibration, road surface irregularities, motion of the sensor within the vehicle, and so on. Therefore, a discrete, fast wavelet transform signal-processing algorithm is applied to the acceleration data to reveal the component frequencies. These isolated frequencies serve as much stronger stimuli for a machine learning algorithm attempting multi-resolution analysis.

\subsubsection{GNU Scientific Library}

There are many variations in the literature on the original wavelet transform (Jensen and Cour-Harbo, 2001). This work implements the wavelet transforms in the wellestablished and open-source GNU Scientific Library (Free Software Foundation, Inc., 2013). The GNU Scientific Library implements the B-spline, Daubechies, and Haar wavelet families. In particular, the GNU Scientific Library implements oneand two-dimensional wavelet transforms based on the biorthogonal B-spline order

$$
(i, j)=(1,3),(1,5),(2,2),(2,4),(2,6),(2,8),(3,1),(3,3),(3,5),(3,7),(3,9)
$$


and Daubechies maximum phase

$$
\frac{k}{2}, k=4,6,8,10,12,14,16,18,20
$$

wavelet families. The simulator sends the acceleration data from each test event through all available wavelet families and members, twenty in all, in order to enable accuracy values from later machine-learning-algorithm training to determine which wavelet transform is best suited for the task of providing intelligent driver feedback.

A third consideration is the amount of computation efficiency required in a proposed smart phone application to process the data and provide feedback to the driver. Simple wavelet transform algorithms require sample sizes that are powers of two (Jensen and Cour-Harbo, 2001). The simulation preserves 512 acceleration data points leading up to each test run. This value is selected as a balance between the discrete wavelet transform requirement of data lengths that are powers of two versus the value being the lower bound of dimensionality for the support vector machine. Thus, the allowed wavelet transforms sizes are 512, 256, 128, 64, 32, 16, $8,4,2$, and 1 . Since the simulations proceed at a time increment of 0.2 seconds, this translates to $102.4,51.2,25.6,12.8,6.4,3.2,1.6,0.8,0.4$, and 0.2 seconds of acceleration history, respectively. Because driver reaction time must be taken into consideration, sample sizes of 16 and fewer clock steps are not considered.

In a real-world implementation, time is continuous and the accelerometer in a smart phone can be sampled at a variety of time scales, dependent on the sensor hardware in the device, but within the range 10Hz-100Hz (Apple, Inc., 2013; Corona Labs Inc., 2014; STMicroelectronics, 2014a,b). However, elapsed time between sam- 
ples cannot be guaranteed, so some combination of oversampling and interpolation may be necessary prior to performing the wavelet transform.

Each $2^{n}, n=5,6,7,8,9$ sample size subset of data from simulation test events is run through each GNU Scientific Library discrete wavelet transform and the results saved, 100 variations derived from each test event generated by the simulator. Each of these variations will be used as input to the machine learning algorithm and the ability of the support vector machine (SVM) to make an accurate prediction will be used to determine both the acceleration data sample size needed and the discrete wavelet transform most capable of producing correct feedback to the driver.

The different transformations of the acceleration data, the binary positive or negative event classification, and the magnitude (weight) of that classification are used as input to the weighted data variant of the support vector machine LIBSVM (Chang and Lin, 2011).

\subsection{Machine Learning}

By the end of its execution, the simulator as configured in this thesis will have generated several thousand test events, each containing a wavelet transform of the sensor vehicle's acceleration data and the effect on macroscopic traffic flow over the time of the sensor vehicle suppressing its own acceleration temporarily. The simulated events will then be used as input for an artificial intelligence machine learning algorithm to attempt to classify good versus bad results from the simulation. 


\subsubsection{LIBSVM}

This thesis makes use of a support vector machine from the well-established (BenHur and Weston, 2010) and open-source LIBSVM (Chang and Lin, 2011). LIBSVM supports one-class and multi-class classification and regression, with a variety of kernels (linear, polynomial, radial basis function, and sigmoid). LIBSVM also implements solution cross-validation which allows one to determine the best parameters to use with the radial basis function kernel, which is recommended by the authors as a good general-purpose kernel:

$$
K\left(\mathbf{x}_{i}, \mathbf{x}_{j}\right)=\exp \left(-\gamma\left\|\mathbf{x}_{i}-\mathbf{x}_{j}\right\|^{2}\right), \gamma>0
$$

As a support vector machine itself has tunable parameters $C$ and $\gamma$, it is necessary to search the parameter space methodically to find the parameters that will produce the best accuracy. The authors of LIBSVM document such a method in a paper accompanying the software library (Hsu et al., 2010). That method is as follows, in brief (Hsu et al., 2010, p. 3):

- Scale data;

- Use the Radial Basis Function (RBF) kernel;

- Find the best combination of values for $C$ and $\gamma$;

- Use the best $C$ and $\gamma$ for the entire training set.

LIBSVM comes in its standard form and also in several variants. One variant supports weighted vectors. Each simulated event can be weighted based on the positive or negative effect it had on traffic flow. As noted in Section 3.3.1, this thesis uses the weighted variant of LIBSVM. LIBSVM itself does not have a parallel-processing 
architecture and could be used in a smart phone application or embedded control system implementing the intelligent driver feedback heuristic.

\subsection{Signal Processing}

The output of each wavelet transform contains all coefficients necessary to reconstruct the original acceleration data. A wavelet transform contains information about the features of a signal, and not the signal itself, in its coefficients. It may be the case that the full wavelet transform contains too much detail for the support vector machine to be able to maximize its solution accuracy. Coefficients with smaller values correspond to less important features. Therefore an arbitrary amount of "noise" is removed from the wavelet transform by setting one or more of the smallest coefficients to zero by removing it from the data file. The Support Vector Machine is retrained using the significant features only and the accuracy compared to that from the complete wavelet transform. 


\section{Chapter 4}

\section{Results}

This chapter reports on the performance of the traffic simulation, the wavelet transforms, and the machine learning algorithm, and signal processing done in this work. The results of all iterations of the support vector machine training are explored and analyzed in detail.

\subsection{Simulation}

The events of multiple simulations recording data from different sensor vehicles on the roadway are detailed in Table 4.1. The table includes:

1. The execution number (run),

2. The ring road length (circumference in kilometers),

3. Which vehicle in the traffic stream was sampled (sensor vehicle number),

4. How many events collected from the sensor vehicle were generated by each run, 


\begin{tabular}{cccccccc}
\hline Run & $\begin{array}{c}\text { Road } \\
\text { Length } \\
(\mathrm{km})\end{array}$ & $\begin{array}{c}\text { Sensor } \\
\text { Vehicle } \\
\text { Number }\end{array}$ & $\begin{array}{c}\text { Test } \\
\text { Events }\end{array}$ & $\begin{array}{c}\text { Total } \\
\text { Clock } \\
\text { Iterations }\end{array}$ & $\begin{array}{c}\text { Real-time } \\
\text { Equivalent } \\
\text { (days) }\end{array}$ & $\begin{array}{c}\text { Simulation } \\
\text { Duration } \\
\text { (hours) }\end{array}$ & $\begin{array}{c}\text { \# of } \\
\text { CPUs }\end{array}$ \\
\hline 1 & 10.0 & 0 & 5,902 & $41,097,466$ & 95.1330 & 5.148 & 4 \\
2 & 10.0 & 95 & 5,645 & $39,814,964$ & 92.1643 & 6.413 & 8 \\
3 & 10.0 & 150 & 5,810 & $40,111,950^{1}$ & $92.8517^{1}$ & - & 4 \\
4 & 10.0 & 111 & 5,859 & $40,258,950$ & 93.1920 & 23.570 & 8 \\
5 & 10.0 & 37 & 6,004 & $40,979,014$ & 94.8588 & 8.630 & 4 \\
6 & 10.0 & 48 & 5,563 & $39,568,9641$ & $91.5948^{1}$ & - & 4 \\
7 & 10.0 & 6 & 6,147 & $41,466,511$ & 95.9873 & 3.740 & 6 \\
8 & 10.0 & 5 & 6,194 & $41,538,512$ & 96.1540 & 4.253 & 5 \\
9 & 10.0 & 2 & 6,155 & $41,499,512$ & 96.0637 & 4.969 & 2 \\
10 & 10.0 & 3 & 6,432 & $41,898,517$ & 96.9873 & 7.671 & 3 \\
11 & 10.0 & 7 & 6,248 & $41,594,011$ & 96.2824 & 3.729 & 7 \\
12 & 10.0 & 8 & 6,465 & $41,912,010$ & 97.0185 & 3.771 & 8 \\
\hline Total & - & - & 72,424 & $491,740,381$ & 1138.29 & 71.894 & - \\
\hline
\end{tabular}

1 estimated

Table 4.1: Results from Different IDM Simulations

5. How many 0.2 second clock iterations elapsed on the master, look-ahead, and test roads combined,

6. The same amount expressed in decimal days, and

7. The particulars of the computational environment.

\subsubsection{Explanation of Collected Data}

Each test event consists of two items: first, an initial condition consisting of the sensor vehicle's (wavelet transformed) acceleration curve leading up to the modified behavior and second, the increase or decrease in traffic flow between the test and control roads over a five-minute period following the temporary change in behavior of the sensor vehicle. (The increase or decrease in kinetic intensity of all vehicles on 
Table 4.2: Descriptive Statistics from All Twelve Simulation Runs

\begin{tabular}{lrl}
\hline Data Property or Statistic & Value & Position \\
\hline Count & 72424 & \\
Minimum percent change flow $(\% \Delta Q)$ & -5.53311 & \\
Maximum $\% \Delta Q$ & 3.50466 & \\
Mean $\% \Delta Q$ & -0.08459 & \\
Median $\% \Delta Q$ & -0.06678 & \\
Standard Deviation $\% \Delta Q$ & 0.68133 & \\
95th percentile $\% \Delta Q$ & 0.99870 & 68,802 \\
96th percentile $\% \Delta Q$ & 1.07816 & 69,527 \\
97th percentile $\% \Delta Q$ & 1.18244 & 70,251 \\
98th percentile $\% \Delta Q$ & 1.33252 & 70,975 \\
99th percentile $\% \Delta Q$ & 1.58095 & 71,699 \\
99.9th percentile $\% \Delta Q$ & 2.35329 & 72,351 \\
99.99th percentile $\% \Delta Q$ & 3.04055 & 72,416 \\
99.999th percentile $\% \Delta Q$ & 3.50466 & 72,423 \\
\hline
\end{tabular}

the road is also recorded, but not used; see Section 2.1.3 and Figure 3.8.) A positive result is taken to be one in which traffic flow increased and a negative result is taken to be one in which traffic flow did not change or decreased. In this way, it is possible to use the shape of the acceleration curve as a determinant of providing intelligent feedback to the driver in order to improve traffic flow.

In the simulation, test events are generated at each clock step when the sensor vehicle has an upcoming velocity drop below 20\% of its desired velocity (Figure 3.1). Additionally, each event was recorded with five different lengths of wavelet transformed acceleration data: 32, 64, 128, 256, and 512 data points; see Section 3.3. 


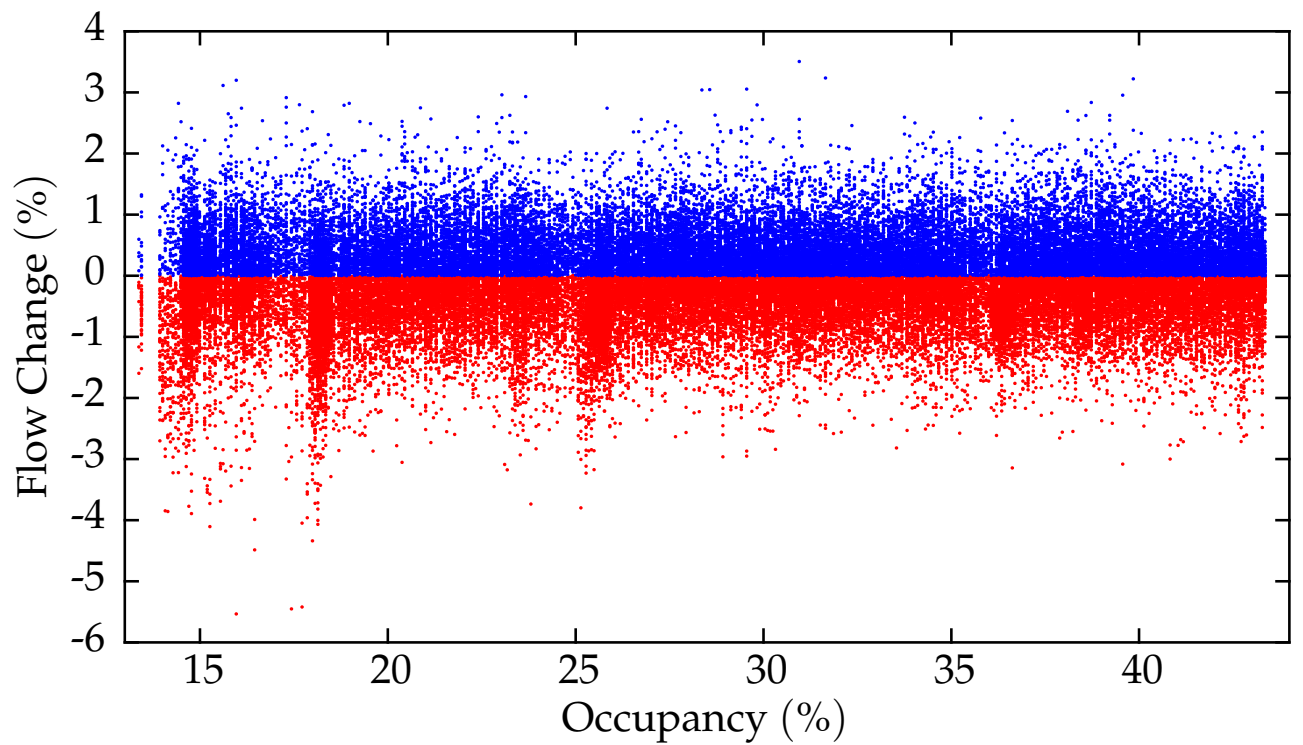

Figure 4.1: Effects of each test on traffic flow across the range of road occupancy simulated.

\subsubsection{General Characteristics of Collected Data}

Table 4.2 provides descriptive statistics from events from all simulations. In particular, an event resulting in a $1.0 \%$ or better improvement in traffic flow on the roadway occurs beginning at the 95th percentile and above. Figure 4.1 shows all test events visually; a positive test event is represented by a blue dot above 0.0 on the y axis; a negative test event is represented by a red dot below 0.0 on the y axis. The ability of the sensor vehicle to affect flow is fairly uniform across all occupancy levels.

Figure 4.2 displays the effect of all test events on traffic flow as a histogram, rounded to the nearest tenth of a percent. The histogram suggests that it is slightly easier to have a negative than positive effect on flow; in fact, $55 \%$ of test events reflect a negative outcome $(n=39,826)$. 


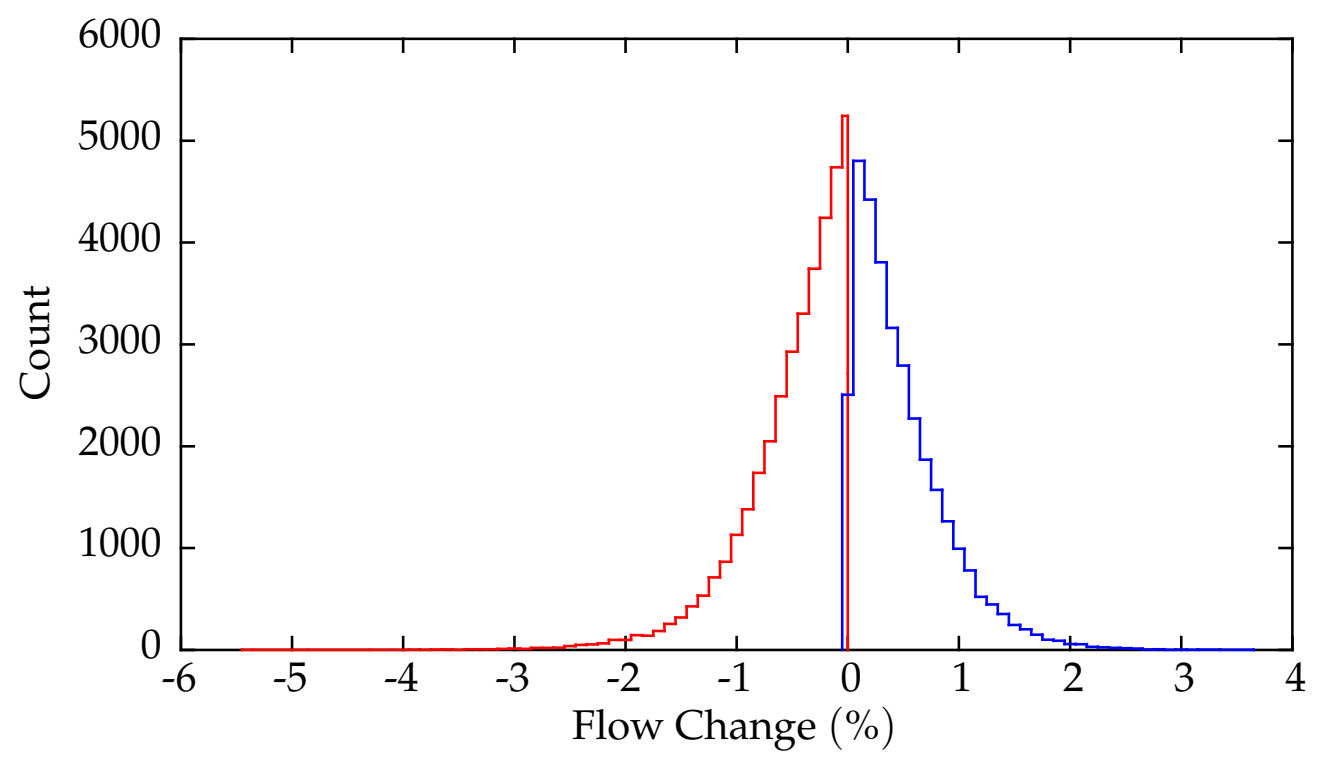

Figure 4.2: Effects of each test on traffic flow summarized as a histogram.

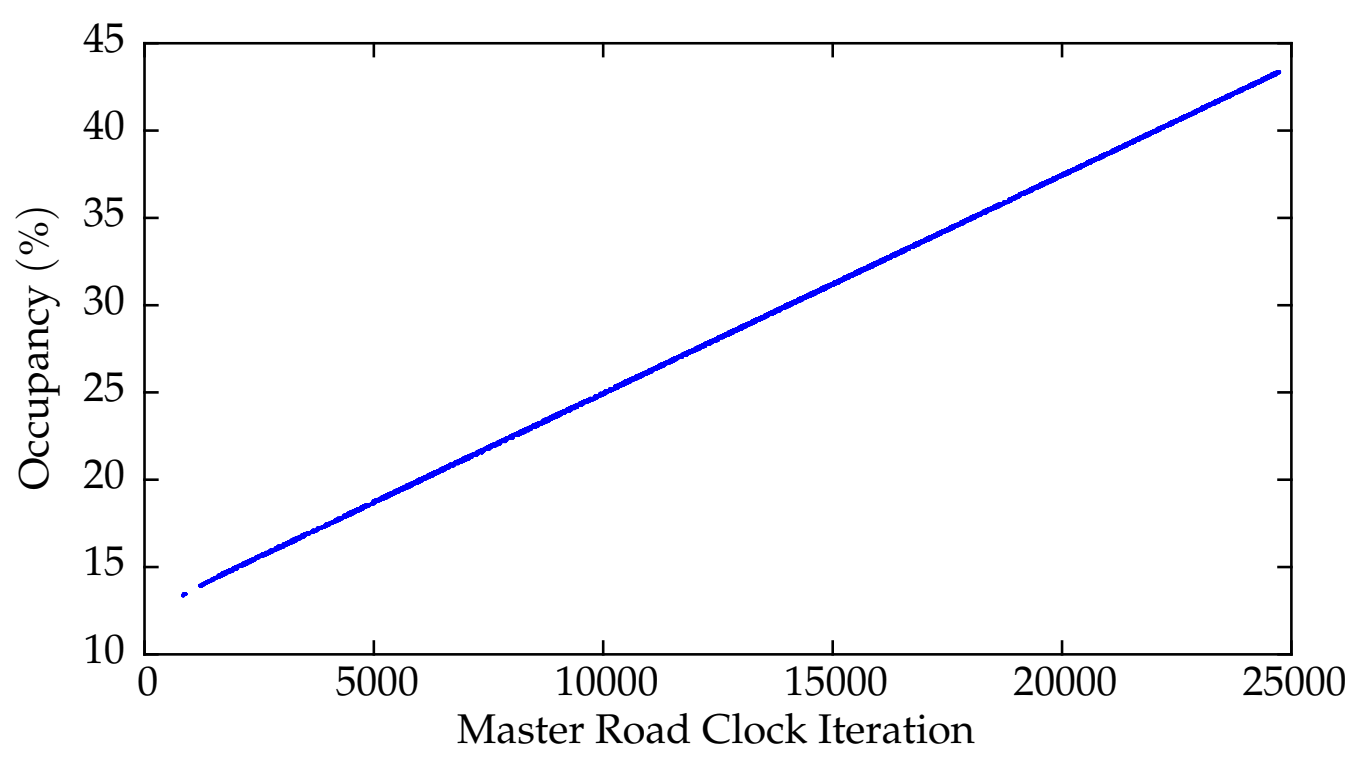

Figure 4.3: Road occupancy as a function of elapsed simulation time. 


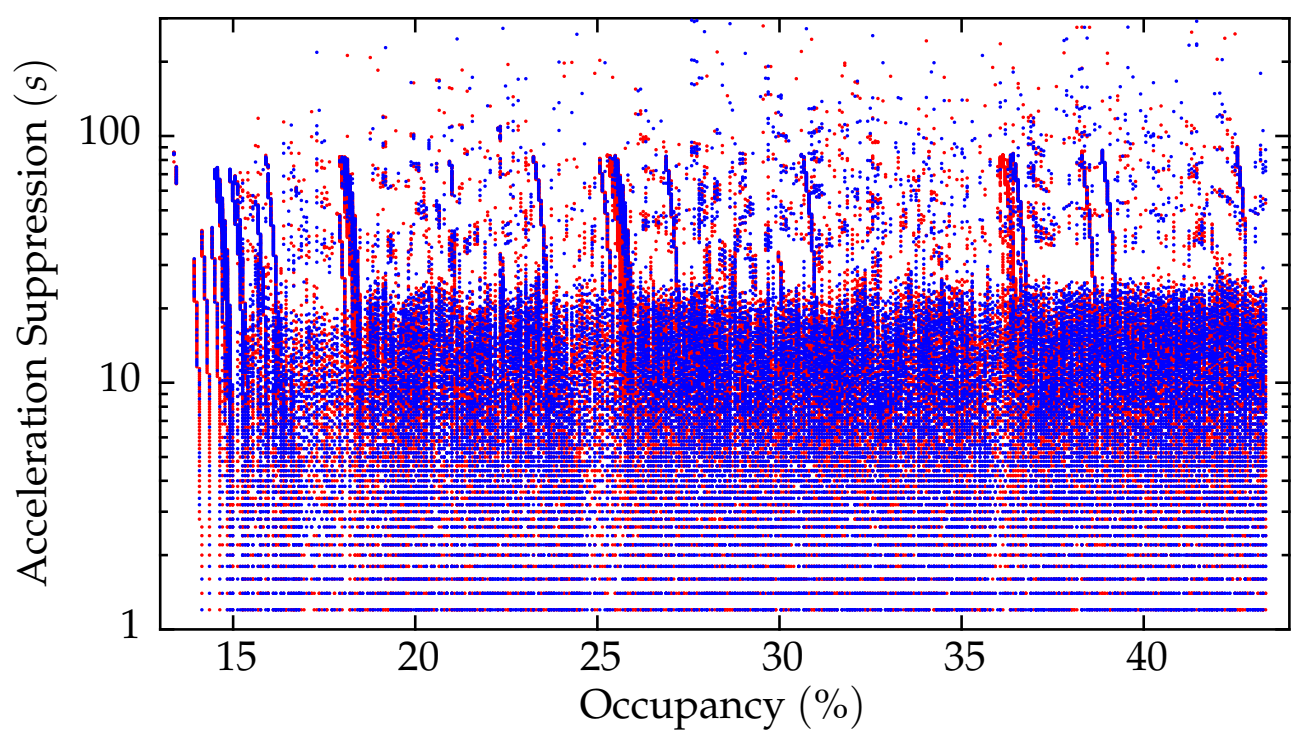

Figure 4.4: Duration of acceleration suppression as a function of road occupancy.

Figure 4.3 illustrates how the simulator is configured to add vehicles to the traffic stream as occupancy increases. The simulator is set to attempt to add a vehicle to the road every 10 seconds in this application (it supports any clock increment greater than 0 ) and, as can be seen in the figure, is able to keep that pace throughout, as confirmed by the linear trend.

Figure 4.4 displays the amount of time that the acceleration of the sensor vehicle is suppressed (velocity maintained) prior to catching up with a velocity shockwave, necessitating braking. Positive test events are shown in blue and negative test events are shown in red (Section 4.1.1). The horizontal banding observed below 10 seconds is due to the time step used in the simulator, 0.2 seconds, as displayed on the logarithmic scale used for the y axis. No pattern in occupancy or duration of acceleration suppression can be visually identified in the figure. The most striking visual feature is the de facto maximum duration of acceleration suppression between 


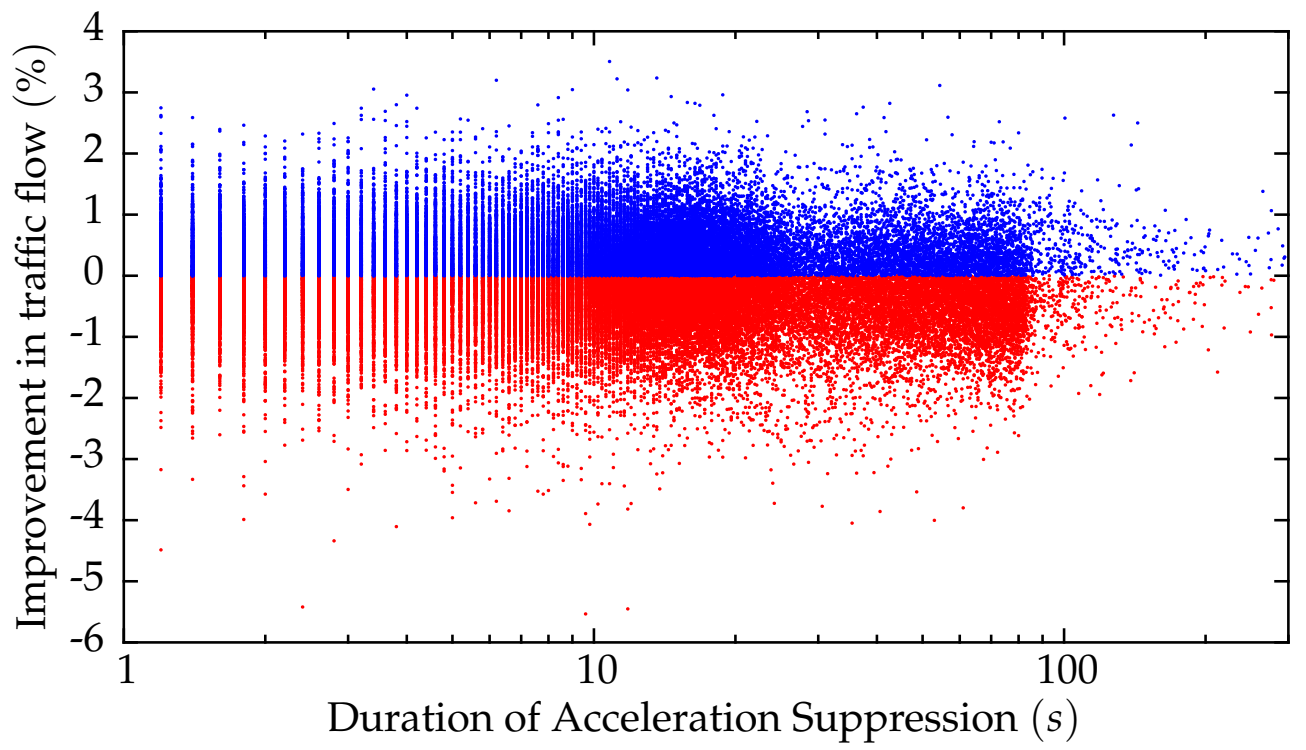

Figure 4.5: Test event effect on traffic flow as a function of the duration of acceleration suppression.

20 and 30 seconds, but the most unusual visual feature is the existence of data points above 102.4 seconds. This is because 102.4 seconds (512 data points) is the maximum duration of the look-ahead when detecting a velocity drop. To have a duration of acceleration suppression longer than 102.4 seconds means that the sensor vehicle was able to completely absorb the downstream velocity shockwave. Unfortunately, these events do not always have a positive effect on traffic flow, are rare enough to be outliers, and are therefore not explored further in this thesis.

Figure 4.5 depicts a scatterplot of the duration of the acceleration suppression by the sensor vehicle and the resulting effect on traffic flow. In this figure, the vertical banding below 10 seconds is due to the time step used in the simulator, 0.2 seconds, as displayed on the logarithmic scale used for the $\mathrm{x}$ axis. Similar features can be observed here as in Figure 4.4, such as the drop in data points above 25 and 85 seconds. 


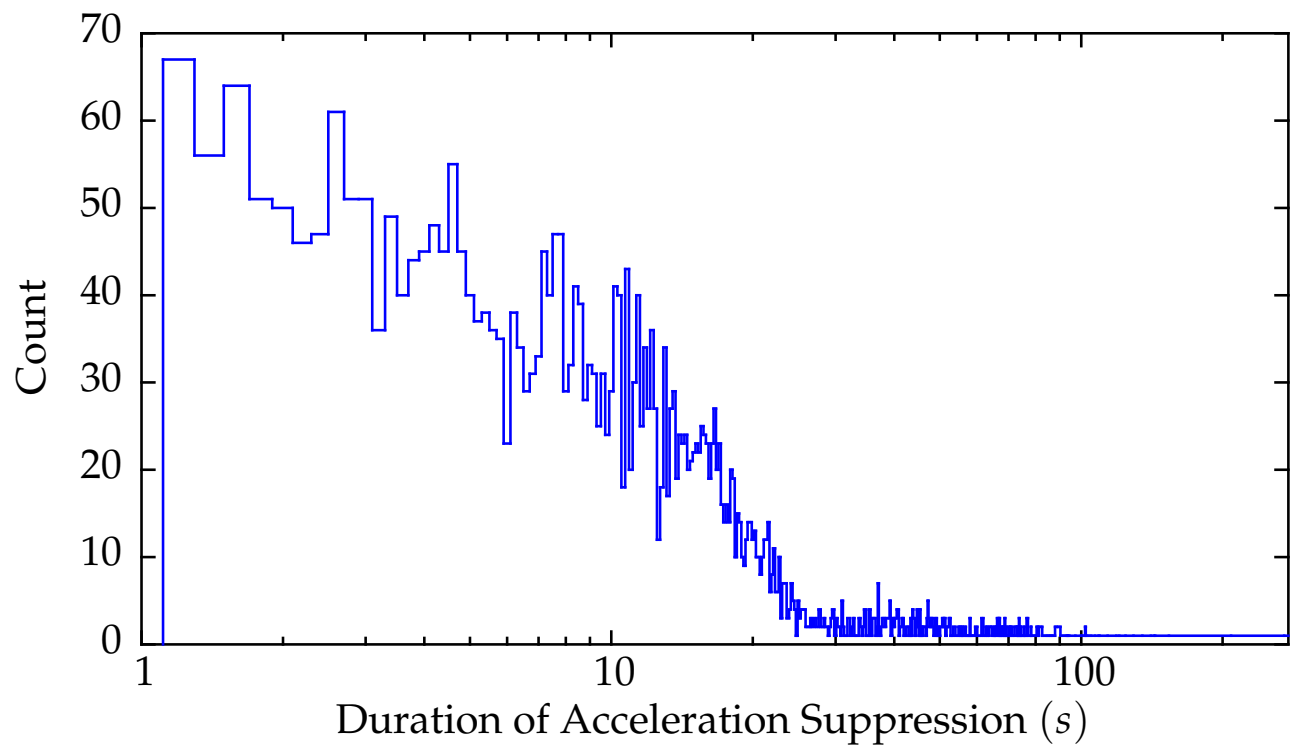

Figure 4.6: Histogram of Acceleration Suppression Duration.

Figure 4.6 shows a histogram of the duration of sensor vehicle acceleration suppression drawn from test events having a $1 \%$ or better effect on traffic flow. Most events have a duration of 20 seconds or less, though there is a long tail extending beyond 200 seconds. This suggests that some upper bound of what constitutes an actionable event could filter the positive events before going to the support vector machine.

Figure 4.7 depicts a histogram of the test events where the sensor vehicle suppressing its acceleration produced an improvement in traffic flow for the entire road greater than $1 \%$. The changes in traffic flow are rounded to the nearest $0.1 \%$ in this figure. Most events have around a $1 \%$ to $1.5 \%$ effect, though a very few substantial effects above $2 \%$ exist. The drop in the count at $1.0 \%$ is an effect of rounding, because tests with an effect of $0.95 \%-1.0 \%$ are not in the set of positive events. 


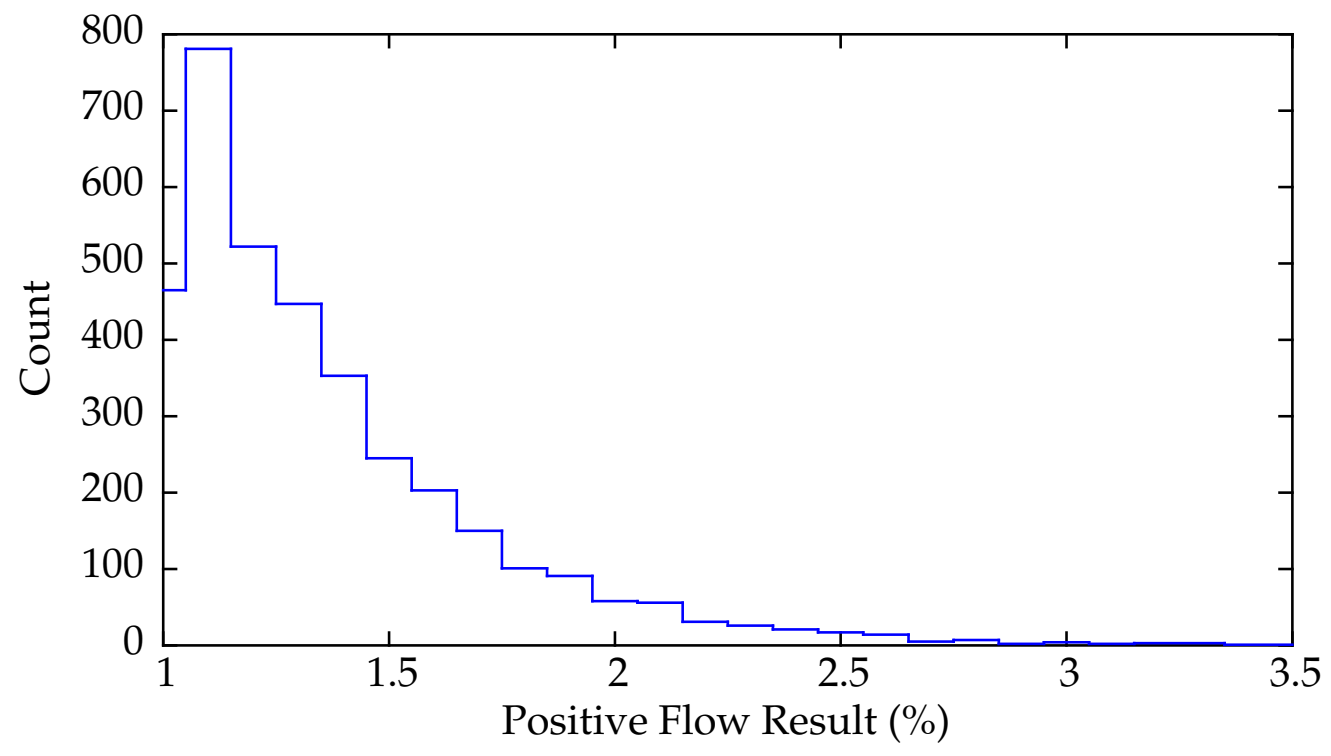

Figure 4.7: Histogram of event flows greater than one percent.

Finally, Figure 4.8 plots duration of acceleration suppression against traffic flow effect. In this figure, the vertical banding below 10 seconds is due to the time step used in the simulator, 0.2 seconds, as displayed on the logarithmic scale used for the $x$ axis. This figure is, in essence, the top portion of Figure 4.5 and shows only the detail of test events with a positive effect on traffic flow in excess of $1 \%$. Similar to Figure 4.6, test events drop off somewhat above 20 seconds' duration of acceleration suppression.

At this point in the analysis it is not possible to isolate any range of occupancy levels as being particularly sensitive to the behavior tested, namely, suspending acceleration in anticipation of an upcoming velocity drop. Statistically, triggering this behavior randomly would have a slightly higher likelihood of having a negative effect on traffic flow on the roadway (55\% to $45 \%$ ). However, the experiment seeks 


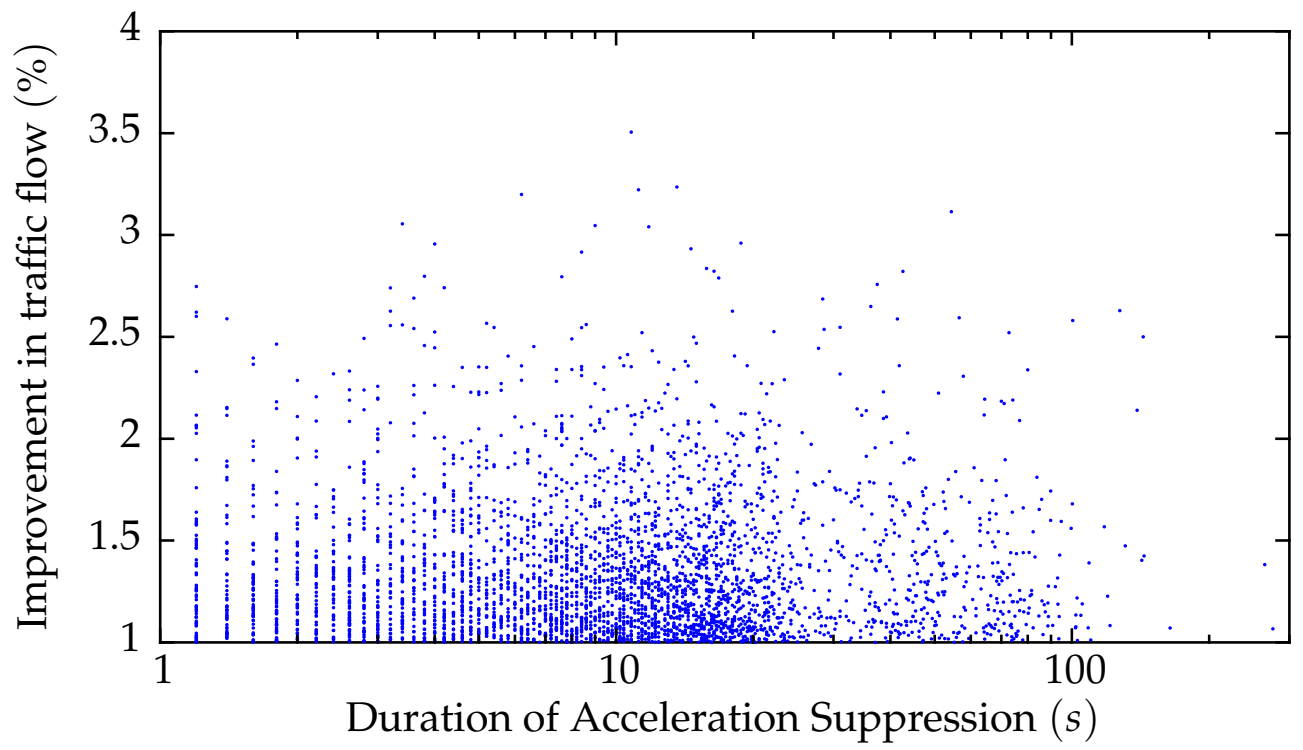

Figure 4.8: Scatterplot of Positive Results vs. Suppression Duration.

to understand the initial conditions required to produce a positive result when triggering the behavior. 


\subsection{Wavelet Transform Performance}

The experiment post-processed each length of acceleration curve using each of twenty discrete wavelet transforms in the GNU Scientific Library and recorded the transformed data along with the associated positive or negative effect on macroscopic traffic flow improvement. The relative performance of each wavelet transform will be compared in the next section in the context of the ability of the support vector machine to accurately classify new events based on its learning from simulator events.

\subsection{Support Vector Machine Calculation}

The goal of this work is to find a local, if not global, maximum of classification accuracy across four dimensions: one of five lengths of acceleration data samples; one of twenty varieties of discrete wavelet transformation; and two parameters required by the support vector machine $-C$, the penalty parameter of the error term, and $\gamma$, a parameter of the radial basis function kernel (Section 3.4.1) (Chang and Lin, 2011). At this point, the standalone support vector machine trainer in the weighted variant of LIBSVM, svm-train, is used to calculate a hyperplane separating positive results (increased traffic flow) from negative results (decreased traffic flow). This work uses an iterative approach to find the best accuracy possible. 


\subsubsection{Support Vector Machine Calculation: First Iteration}

This iteration processes the events from simulator runs number 1 and 2 (Table 4.3) using each of the five sample lengths and 20 discrete wavelet transforms for a total of 100 possible support vector machines. The intent of this broad search is to discard from further consideration poorly performing wavelet transform and sample length combinations based on the classification accuracy of support vector machines trained using those poor combinations.

Additionally, a Support Vector Machine using a Gaussian Radial Basis Function kernel uses two adjustable parameters, $C$ and $\gamma$. The LIBSVM cross-validating solution space search script easy . py spawns instances of svm-train, using values of $C$ over the range $\log _{2} C=-5,-3, \ldots, 15$ and $\gamma$ over the range $\log _{2} \gamma=-15,-13, \ldots, 3$ for a total of 110 trials (Figure 4.9) per combination of sample length and wavelet transform in order to determine parameter values that will produce the highest classification accuracy ("best-in breed"). Svm-train uses $75 \%$ of the 11,547 events as the training corpus $(n=8,661)$, and the final $25 \%$ for the testing corpus $(n=2,886$; Table 4.3).

The experiment did not calculate all 100 possible "best-in-breed" support vector machines due to an interruption in power to the computers used. The experiment was not restarted owing to the poor accuracy observed overall. The lack of variation in accuracy between sample lengths and wavelet transform families and members is surprising and requires further investigation. No firm conclusion about which time window sample sizes or wavelet transforms work best can have a significant impact on traffic flow can be inferred from these results. 


\begin{tabular}{|c|c|c|c|c|c|c|}
\hline Sample Length & DWT Family & 32 & 64 & 128 & 256 & 512 \\
\hline B-spline 1,3 & & 53.7929 & 54.0238 & 54.0584 & 54.1854 & - \\
\hline B-spline 1,5 & & 53.6543 & 54.2085 & 54.1739 & 54.2663 & - \\
\hline B-spline 2,2 & & 53.7582 & 53.5735 & 53.6659 & 53.8390 & - \\
\hline B-spline 2,4 & & 53.7582 & 53.7120 & 53.6428 & 53.7236 & - \\
\hline B-spline 2,6 & & 53.7120 & 53.7005 & 53.5966 & 53.8044 & - \\
\hline B-spline 2,8 & & 53.7351 & 53.8621 & 53.6659 & 53.7467 & - \\
\hline B-spline 3,1 & & 53.6890 & 53.6659 & 53.6774 & 53.6543 & - \\
\hline B-spline 3,3 & & 53.7929 & 53.7351 & 53.6890 & - & - \\
\hline B-spline 3,5 & & 54.0122 & 53.7467 & 53.6543 & - & - \\
\hline B-spline 3,7 & & 53.3888 & 53.7120 & 53.6543 & - & - \\
\hline B-spline 3,9 & & 53.7813 & 53.6774 & 53.6197 & - & - \\
\hline Daubechies 6 & & 53.4349 & 53.7236 & - & - & - \\
\hline Daubechies 8 & & 53.6543 & 53.7582 & - & - & - \\
\hline Daubechies 10 & & 54.1162 & 53.7120 & - & - & - \\
\hline Daubechies 12 & & 54.0122 & 53.7929 & - & - & - \\
\hline Daubechies 14 & & 53.9199 & 53.7929 & - & - & - \\
\hline Daubechies 16 & & 53.8044 & 53.9083 & - & - & - \\
\hline Daubechies 18 & & 53.9083 & - & - & - & - \\
\hline Daubechies 20 & & 53.9083 & - & - & - & - \\
\hline Haar 2 & & 53.7813 & - & - & - & - \\
\hline
\end{tabular}

Table 4.3: Best percent accuracy predicting testing data across all values of $C$ and $\gamma$. 
Figure 4.9 shows an example of results from LIBSVM using the data from simulator runs 1 and 2. The figure displays topographic contours at fixed height intervals of the result surface generated by invoking the Support Vector Machine calculation with different values of $C$ and $\gamma$. The surface height is the classification accuracy (\%) on the training corpus. LIBSVM's easy . py script implements the following approach:

1. svm-scale to perform data scaling;

2. grid.py to iterate over the parameter space for $C$ and $\gamma$, repeatedly calling svm-train to actually generate a support vector machine;

3. svm-predict to calculate model accuracy against the testing corpus; and

4. gnuplot to visualize the results generated.

The figure generated by gnuplot is a contour plot showing the results within the context of neighboring parameters. In the example, there are two local maxima above $52.5 \%$. It is possible to specify smaller parameter ranges and step sizes to narrow the parameter search to the most promising areas.

\subsubsection{Support Vector Machine Calculation: Second Iteration}

Given the disappointing but nearly identical accuracy of all discrete wavelet transform variations and different amounts of wavelet-transformed acceleration data, this iteration focuses only on a Haar 2 wavelet transform of 32 acceleration data points. However, this change is not to address the disappointing support vector machine classification accuracy. Instead, the experiment reevaluates what constitutes a positive classification.

When the simulation generates a test event, it classifies the event based on whether the test road's macroscopic traffic flow measurement increases (class +1 ) 
Best accuracy on training corpus $=52.9038 \%$

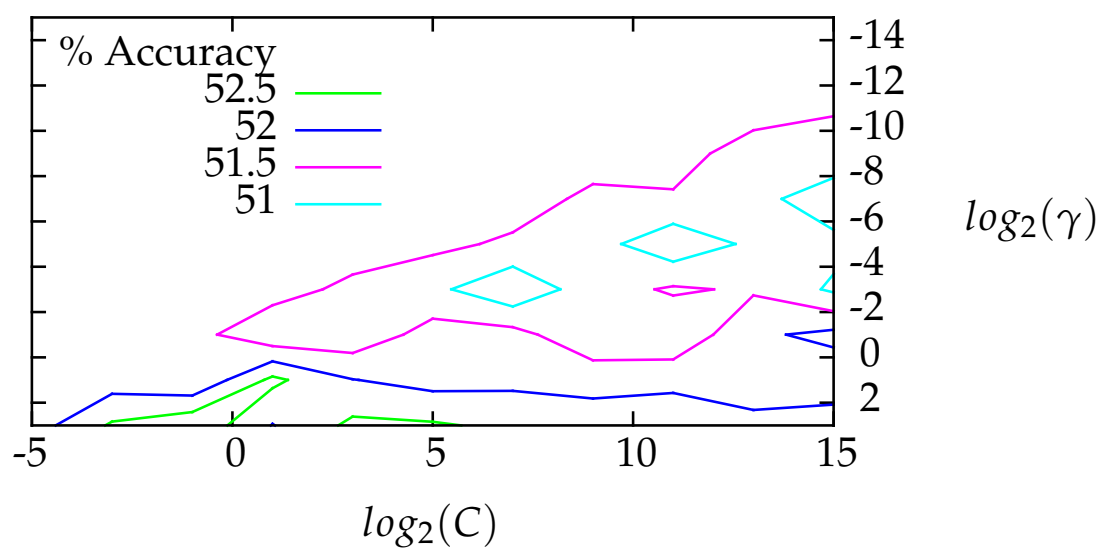

Figure 4.9: Visual display of support vector machine accuracy over ranges of parameters.

or decreases (class -1) compared to the control road. Any non-negative difference, however small, is considered a positive result, and any negative difference is considered a negative result. However, the goal of this work is to make a measurable difference in congested traffic flow. Therefore, the second iteration biases results so that only those that have at least a one percent improvement in flow on the test road over the control road are classified positive. In fact, a statistical analysis of the data used to train and test the support vector machine $(n=11,547)$ shows a one percent improvement of traffic flow occurring at the 88th percentile and above of ranked events (Table 4.4).

As before, the easy .py script walks the solution space, but only against the Haar 2 wavelet transform of the 32-sample acceleration data. Figure 4.10 shows the results from LIBSVM using the data from simulator runs 1 and 2 biased to 
Table 4.4: Descriptive Statistics from Simulation Runs 1 and 2

\begin{tabular}{lrr}
\hline Data Property or Statistic & Value & Position \\
\hline Count & 11547 & \\
Minimum percent change flow $(\% \Delta Q)$ & -5.53311 & \\
Maximum $\% \Delta Q$ & 3.22185 & \\
Mean $\% \Delta Q$ & -0.08040 & \\
Median $\% \Delta Q$ & -0.04997 & \\
Standard Deviation $\% \Delta Q$ & 0.93846 & \\
88th percentile $\% \Delta Q$ & 0.99033 & 10,161 \\
89th percentile $\% \Delta Q$ & 1.03511 & 10,276 \\
\hline
\end{tabular}

classify only events with a $1 \%$ or higher effect on traffic flow into the +1 class. The classification accuracy is very good across the entire support vector machine parameter search space, in excess of $87 \%$, and only dropping as the cost of the error term $C$ becomes quite large. The uniform performance remains unexpected, however, requiring deeper exploration. Support vector machines are recalculated with additional data from other simulator runs in an attempt to reveal why the support vector machine produces nearly identical classification accuracies over such a wide range of parameters.

\subsubsection{Support Vector Machine Calculation: Third Iteration}

The third iteration of SVM training includes data from all twelve simulator runs, $n=72,424$ (Table 4.1). Simulator events are again biased so that only events with a $1 \%$ or higher positive effect on traffic flow are classified as positive (Table 4.2). LIBSVM easy . py cross-validated accuracy improves with the additional data to $95 \%$ (Figure 4.11), but the unusually uniform accuracy remains. 
Best accuracy on training corpus $=87.7843 \%$

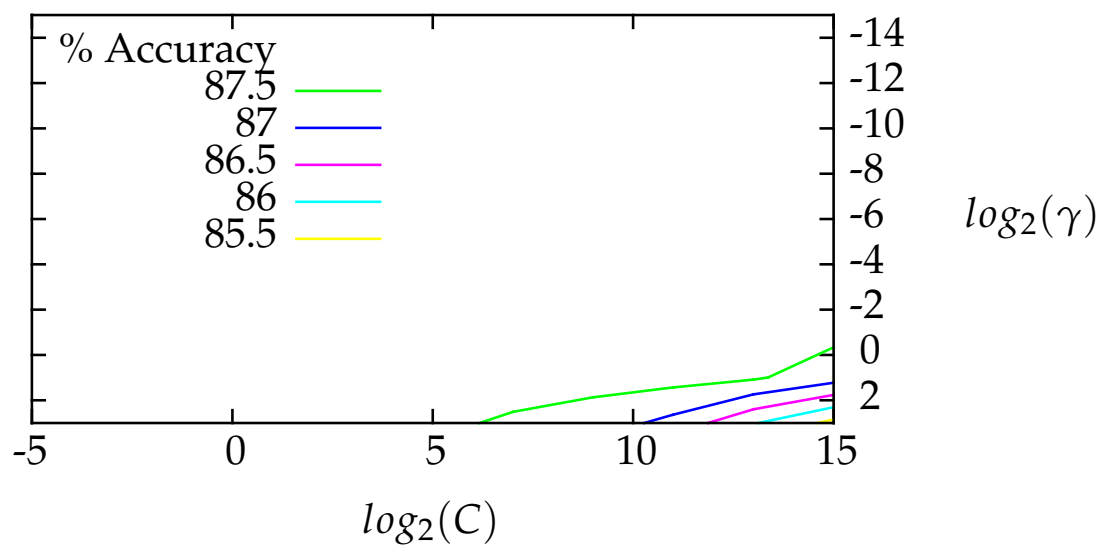

Figure 4.10: Visual display of support vector machine accuracy over ranges of parameters using the 32-sample Haar 2 discrete wavelet transform.

Best accuracy on training corpus $=95.0145 \%$

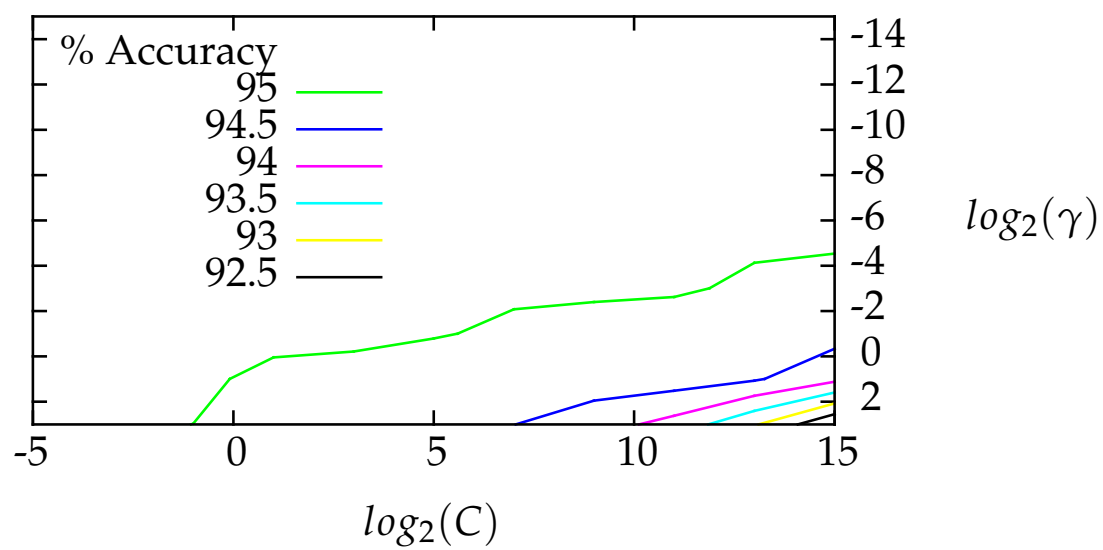

Figure 4.11: Results from LIBSVM using the data from all simulator runs biased to classify only events with a $1 \%$ or higher effect on traffic flow into the +1 class. 


\section{Classification Failures}

The poor and nearly uniform classification accuracy results from the first iteration of support vector machine calculations and nearly uniform classification accuracy results across the parameter space from the second and third iterations invites skepticism. Carefully evaluation of the svm-predict results reveals that the classification of every result in the testing corpus was -1 for many discrete wavelet transform types and across many parameter spaces. LIBSVM silently failed, in that it adopted a majority-class classifier behavior, that is, classified all vectors into the -1 class. This explains the calculated accuracy in many of the results, because the distribution between classes -1 and +1 is $55 \%$ and $45 \%$, respectively, in the first iteration, $88 \%$ and $12 \%$ in the second iteration, and $95 \%$ and $5 \%$ in the third iteration. Therefore, classifying all test data as -1 will guarantee accuracy equal to the percentage population of the -1 class. The majority-class classifier behavior is due to unbalanced data (Ben-Hur and Weston, 2010).

To address this, the misclassification costs change according to this formula (Ben-Hur and Weston, 2010):

$$
\frac{\text { Pos. examples soft }- \text { margin constant }}{\text { Neg. examples soft }- \text { margin constant }}=\frac{\text { Number of neg. examples }}{\text { Number of pos. examples }}
$$

or

$$
\frac{C_{+}}{C_{-}}=\frac{n_{-}}{n_{+}}=\frac{10195}{1352} \approx \frac{7.54}{1}
$$

Based on the relative frequency of classes in the simulation events used in the first iteration, the training and testing corpora are biased so that only events improving 
traffic flow by $1 \%$ or greater are classified +1 . A misclassification cost of 7.54 is used for the +1 class for the remainder of the support vector machine calculations.

At this point, support vector machine calculation restarts with two important modifications to the approach: classifying only the best events into the +1 training and testing corpora, and adjusting the +1 class misclassification cost to address unbalanced data.

\subsubsection{Support Vector Machine Calculation: Fourth Iteration}

Having reclassified events to prefer better outcomes, and having adjusted misclassification costs to address unbalanced data, all varieties of wavelet transform, acceleration data time window, and support vector machine parameter are recalculated. The best accuracy support vector machine for each wavelet transform and acceleration data time window appear in Table 4.5 and graphically in Figure 4.12. Figure 4.12 is commonly known as a "heat map" where different shades along a gradient of colors represent different numeric values. Each cell of the figure represents the classification accuracy for the associated wavelet transform and acceleration data history time window. Shortcomings of the 32-data point time window and Bspline 1,3 and 1,5 and Haar 2 discrete wavelet transforms are immediately apparent. The clearly superior results from the 512-data point time window also stand out.

Two observations can be drawn from these results. First, the shortest acceleration data time windows (32-sample, 6.4-second corpora) perform the worst overall, and the longest acceleration data time windows (512-sample, 102.4 second corpora) perform the best overall. Second, two families of support vector machine perform poorly overall (Haar 2 and B-spline 1,j). The fairly consistent performance of B- 


\begin{tabular}{|c|c|c|c|c|c|c|}
\hline Sample Length & DWT Family & 32 & 64 & 128 & 256 & 512 \\
\hline B-spline 1,3 & & 85.4816 & 83.8184 & 81.3583 & 82.2245 & 85.8281 \\
\hline B-spline 1,5 & & 85.6202 & 3.1254 & 1.2543 & 2.2592 & 5.9667 \\
\hline B-spline 2,2 & & 84.6500 & 86.4865 & 87.5953 & 88.4269 & 88.3576 \\
\hline B-spline 2,4 & & 84.6154 & 86.0360 & 87.4220 & 88.4962 & 88.2190 \\
\hline B-spline 2,6 & & 84.2689 & 86.5904 & 87.5606 & 88.3922 & 88.2190 \\
\hline B-spline 2,8 & & 84.2342 & 85.9321 & 87.5260 & 88.4962 & 88.2536 \\
\hline B-spline 3,1 & & 88.2536 & 86.4172 & 85.7588 & 85.7935 & 89.3624 \\
\hline B-spl & & 84.6847 & 86.4172 & 85.8628 & 85.5856 & 89.3971 \\
\hline B-sh & & 4.5807 & 86.6944 & 85.6549 & 85.6895 & 89.3624 \\
\hline B-s] & & 84.6500 & 86.5 & 86.1 & 85.8628 & 89.3624 \\
\hline B-sp & & 61 & 86 & 8 & 49 & 87.6299 \\
\hline Dau & & 5.1351 & 86.8 & 86.4 & 85.7588 & 86.7983 \\
\hline Daub & & 85.2044 & 86.5211 & 86.1400 & 85.6549 & 89.3278 \\
\hline Daub & & 84.2342 & 86.4865 & 85.6202 & 85.6895 & 89.2585 \\
\hline Daub & & 83.6105 & 86.4518 & 85.8281 & 87.3181 & 88.7387 \\
\hline Daub & & 83.4373 & 86.1400 & 85.9321 & 86.1400 & 88.5308 \\
\hline Daul & & 83.6452 & 86.6251 & 86.4865 & 86.4172 & 88.7734 \\
\hline Dauk & & 83.4719 & 86.3825 & 86.1400 & 87.5953 & 89.3624 \\
\hline Daubechies 20 & & 83.6105 & 86.3132 & 86.0707 & 86.3825 & 89.2931 \\
\hline & & 084 & & 81.2197 & 82.2245 & 85.9667 \\
\hline
\end{tabular}

Table 4.5: Best percent accuracy classifying support vector machine testing corpora across all values of $C$ and $\gamma$. The five highest accuracies are in bold type. 


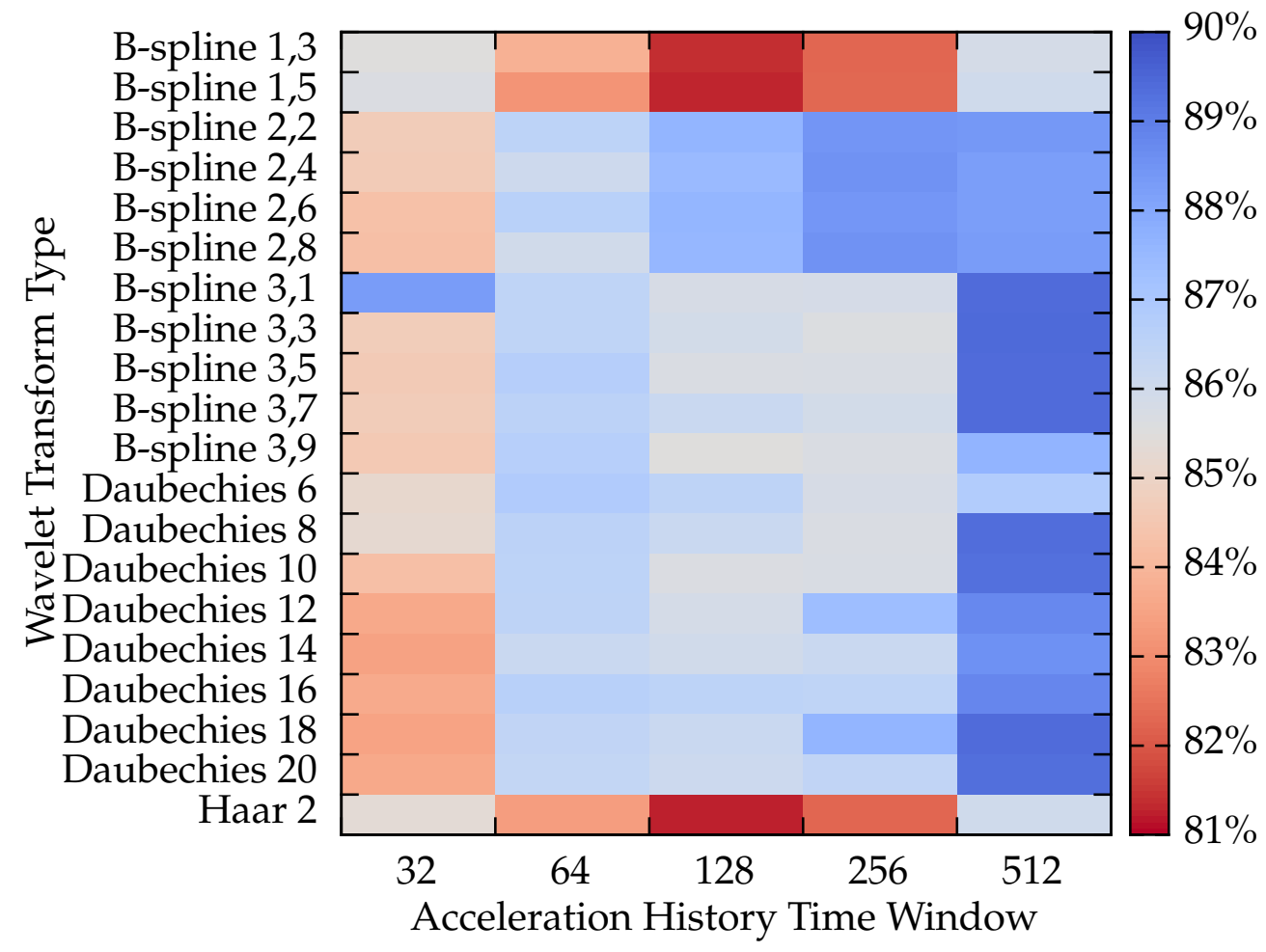

Figure 4.12: Numeric data from Table 4.5 as a heat map for visual interpretation. 
spline $2, j$ notwithstanding, a longer acceleration history is preferable over the use of a particular wavelet transform most of the time.

In an effort to improve classification accuracy above $89.4 \%$, a simple method of noise reduction signal processing now begins using the best overall acceleration data time window, 512 data points, and wavelet transform, B-spline 3,3. Additionally, misclassification costs are adjusted to reflect the relative frequency of +1 and -1 classes across all 12 simulator runs

$$
\frac{C_{+}}{C_{-}}=\frac{n_{-}}{n_{+}}=\frac{68819}{3605} \approx \frac{19.09}{1}
$$

in order to avoid repeating the unbalanced data errors described in Section 4.3.3, and also to use misclassification costs that accurately describe the data from all simulator runs used in the remainder of the analysis.

\subsection{Signal Processing}

A methodical search across the applicable parameter spaces has found a class of discrete wavelet transform that produces superior results and established that a longer acceleration history is necessary for classification accuracy. The final refinement attempts to improve accuracy by removing less important features from the wavelet transform of the acceleration data. Table 4.6 shows the results of training the support vector machine using a B-spline 3,3 wavelet transform of an acceleration data history that is 512 samples at 0.2 seconds per sample (102.4 seconds). All but the largest $n$ coefficients in the wavelet transform have been set to zero. Classification accuracy by the support vector machine improves over the 


\begin{tabular}{lcccc}
\hline Coefficients Preserved (Removed) & $32(480)$ & $64(448)$ & $96(416)$ & $128(388)$ \\
\hline Best accuracy on training data & $85.699 \%$ & $89.4308 \%$ & $91.7762 \%$ & $92.4427 \%$ \\
Best accuracy on testing data & $91.2681 \%$ & $94.2174 \%$ & $93.6043 \%$ & $93.2509 \%$ \\
\hline
\end{tabular}

Table 4.6: Support vector machine classification accuracy given a signal with smaller signal feature coefficients removed (noise reduced).

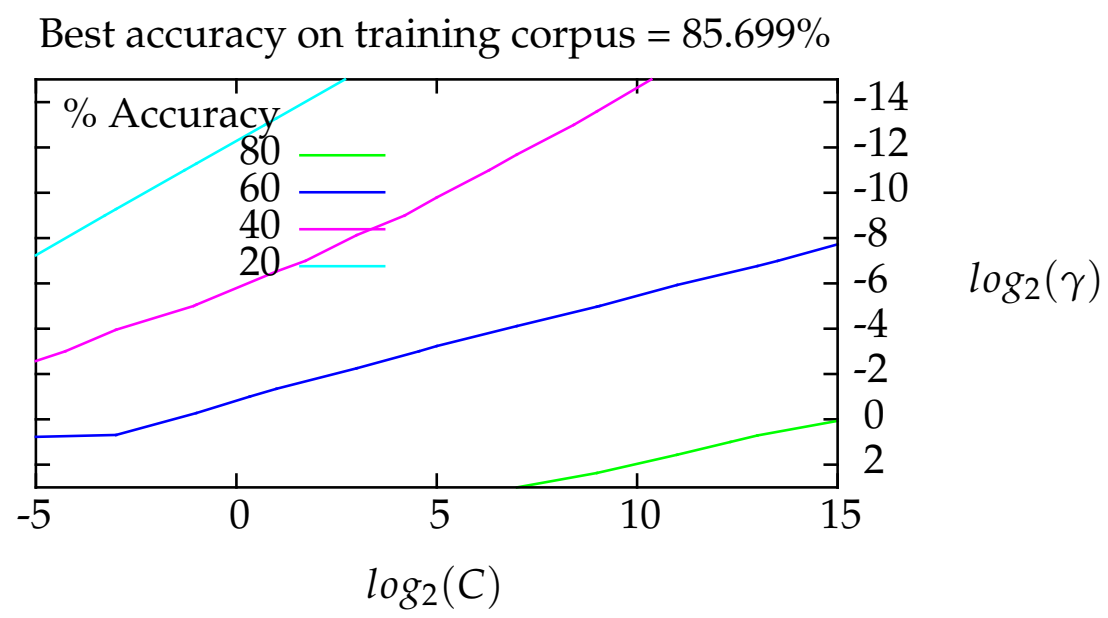

Figure 4.13: Contour map of Support Vector Machine accuracy given signals with the 480 smallest features removed.

original wavelet transform even though more than $80 \%$ of the feature coefficients of the signal have been removed. Optimal noise reduction of the wavelet transform would appear to lie somewhere in the neighborhood of the strongest 64 to 96 signal features. 
Best accuracy on training corpus $=89.4308 \%$

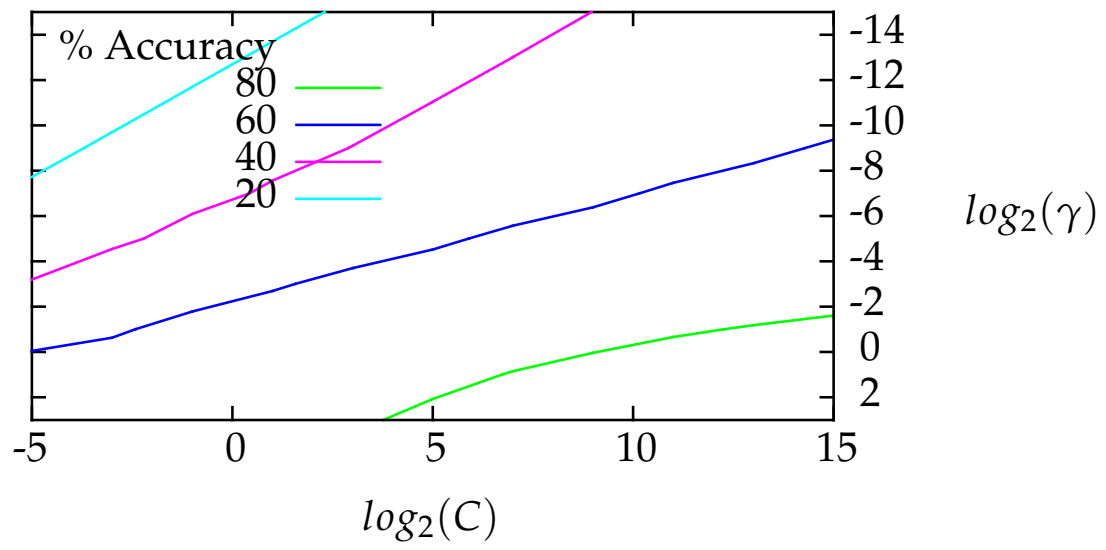

Figure 4.14: Contour map of Support Vector Machine accuracy given signals with the 448 smallest features removed.

Best accuracy on training corpus $=91.7762 \%$

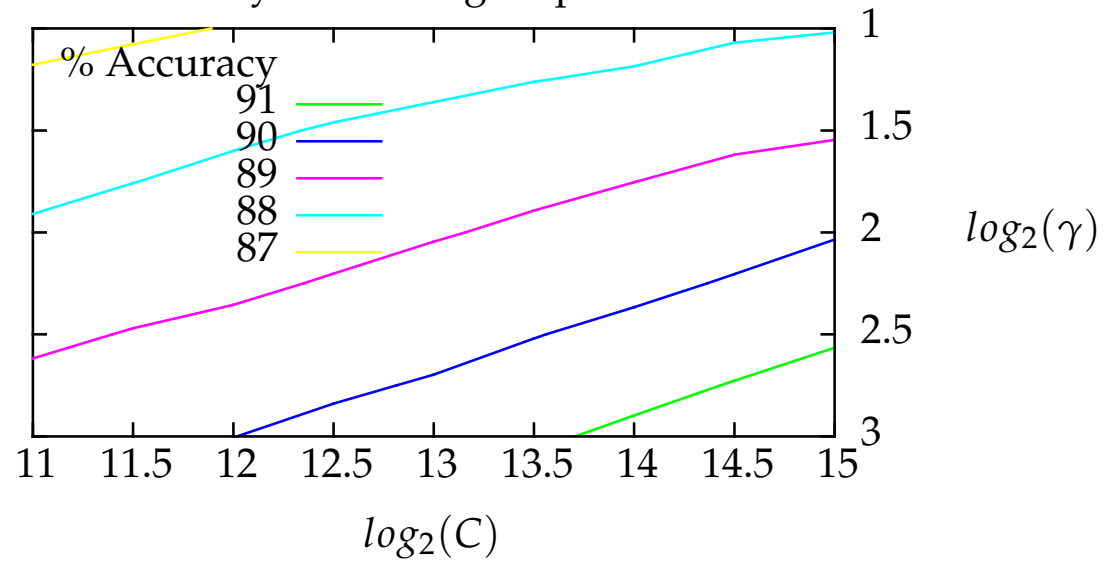

Figure 4.15: Contour map of Support Vector Machine accuracy given signals with the 416 smallest features removed. 


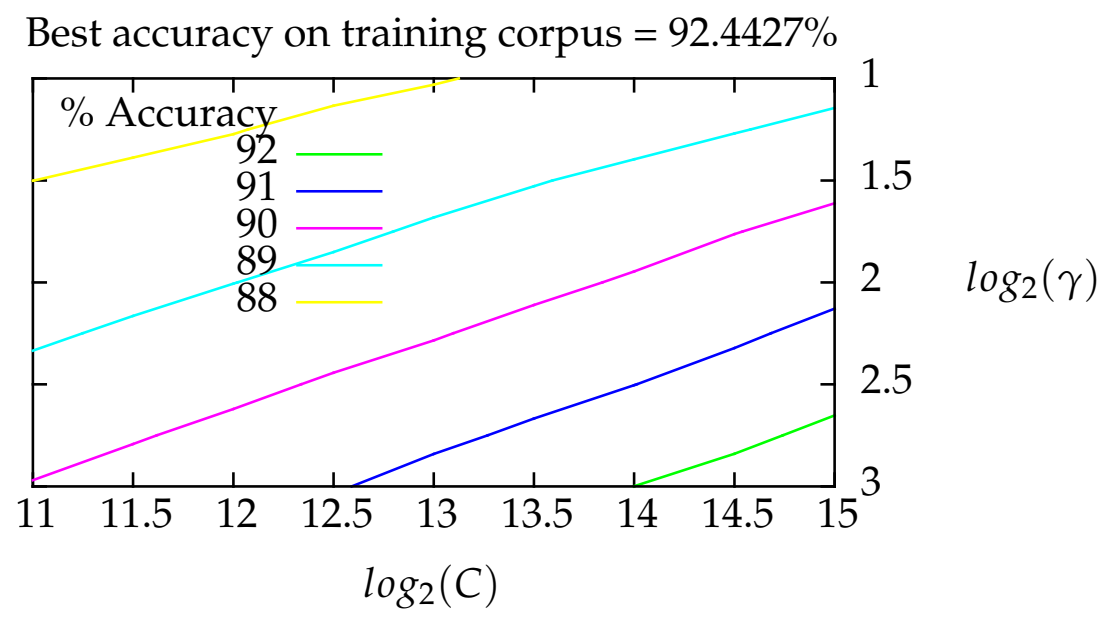

Figure 4.16: Contour map of Support Vector Machine accuracy given signals with the 384 smallest features removed.

\subsection{Summary}

This chapter has described the process of simulating an urban freeway, generating and evaluating test events, with the goal of creating an artificially intelligent heuristic that can distinguish desirable driver behavior events from undesirable ones. At this point in the ongoing research, 12 simulator runs have generated 72,424 test events using a simple freeway simulator with a proven vehicle dynamics model across a wide range of traffic conditions. Each event consists of two things: initial conditions (a longitudinal acceleration signal) and a test result (increase or decrease in traffic flow). The initial conditions are transformed using a Discrete Wavelet Transform to extract features from the signal. Events are classified by desireability of outcome and used to train a heuristic in the form of a Support Vector Machine. 
The heuristic could then be used to provide intelligent feedback, at a later time, to a driver that will allow the driver to mitigate congestion in urban freeway settings via a smart phone application or embedded vehicle system. The heuristic can detect, with greater than $94 \%$ accuracy, opportunities for a driver to take action that will result in increased traffic flow. 


\section{Chapter 5}

\section{Conclusion}

\subsection{Summary}

This thesis attempts to identify conditions in a traffic stream leading up to a velocity drop due to velocity shockwaves in contested traffic. The intent of identifying these conditions is to provide intelligent feedback to a driver or autonomous system. The driver or autonomous system may then take measures to dampen the shockwave, improving traffic flow for the individual vehicle and following vehicles. Introducing this destructive interference into the shockwave works against the emergent, selforganizing, self-amplifying nature of stop-and-go traffic.

In order to develop an intelligent driver feedback heuristic, a microscopic traffic model simulation is programmed. This simulation is structured to detect traffic stream velocity drops, conduct experiments measuring traffic flow based on a change in behavior premise by a sensor vehicle, and collect acceleration data prior 
to the change in behavior and the measure of traffic flow change resulting from the change in behavior. Because little is known about the amount of vehicle history required to make an accurate prediction, multiple lengths of acceleration data history are kept for separate analysis.

Raw acceleration data are merely streams of numbers. This thesis seeks to extract information from those data. Therefore, the acceleration data are processed using a discrete wavelet transformation to extract oscillation features from the signal. There are many different families of discrete wavelet transforms, each with many members. All data generated by the simulations are processed with multiple types of discrete wavelet transforms in order to determine the wavelet transform best suited for this application.

Test events from the simulations are classified according to their impact on traffic flow. Only events at the 95th percentile and above, corresponding to an improvement on traffic flow of at least $1 \%$, are classified as a desirable outcome. The remainder of events are classified as undesirable outcomes. These classified events, with their effect on traffic flow and associated discrete wavelet transformed acceleration data, are then passed along to a support vector machine learning algorithm as training and testing corpora.

Support vector machines are calculated across a range of operational parameters and cross-validated to ensure the best possible classification accuracy. Results show that more acceleration data history produces higher support vector machine classification accuracy. Also, the B-spline 3, $j$ family of support vector machines produces somewhat higher support vector machine classification accuracy for this application than other families of support vector machine. 
Noise reduction is speculatively performed on the wavelet transformed acceleration data signal to remove smaller features of the signal. An improved classification accuracy is observed even when more than $80 \%$ of the coefficients of the wavelet transform are missing.

The final result of this work is a heuristic in the form of a support vector machine model. This heuristic can identify conditions in the traffic stream suitable for providing positive feedback to an individual driver or vehicle with $94.2 \%$ accuracy. Acting on that feedback by ceasing acceleration and maintaining velocity until braking is needed will cause the driver to counteract congestion shockwaves, improving traffic flow on the entire roadway by $1 \%$ to $3.5 \%$ over a subsequent five-minute period.

\subsection{Opportunities to Extend this Work}

There is a long road between this work and implementation in a smart phone application or embedded system in a driver assistance or autonomous vehicle system, due to the gap between a simulated environment and real conditions. For example, the work will need to be validated using a multi-lane microscopic traffic model, where vehicles in adjacent lanes could undermine the goal of the heuristic by entering the current lane. Several ways in which the work can be extended to better approximate real conditions are listed below (Table 5.1). 


\begin{tabular}{ll}
\hline Current Implementation & Possible Improvement \\
\hline Intelligent Driver Model & $\begin{array}{l}\text { Human Driver Model } \\
\text { (Treiber et al., 2006a) }\end{array}$ \\
Single lane roadway & $\begin{array}{l}\text { Multi-lane MOBIL Model } \\
\text { (Kesting et al., 2007a) } \\
\text { Stochastic model }\end{array}$ \\
Deterministic model & Real-world road network/open system \\
Ring road/closed system & Heterogeneous vehicles and dynamics \\
Homogeneous vehicles and dynamics & Noisy/missing acceleration data \\
Noiseless acceleration data & Longer acceleration history, \\
perhaps 819.2 or 1638.4 seconds
\end{tabular}

Table 5.1: Feature-by-feature comparison of implemented concepts and possible replacement technologies that would better approximate real-world conditions. 


\subsection{The Path to Implementing Intelligent Driver Feed- back}

Once a heuristic is finalized, a variety of supporting features are required:

1. Ability to reorient data from the accelerometer (Bhoraskar et al., 2012).

2. Ability to determine smart phone (vehicle) position on the earth in a batteryfriendly way (Ben Abdesslem et al., 2009).

3. Ability to determine that the vehicle is on a highway (map matching).

4. Implementation on smart phone platforms (Apple iOS, Google Android, Microsoft Windows Phone, Mozilla Firefox OS, etc.).

5. Ability to detect if feedback was acted upon, and subsequent reporting to the driver and reinforcement of the behavior.

\subsection{Opportunities after Implementation}

It is the goal of this work to have a tool for intelligent driver feedback which at the very least benefits a single vehicle acting alone in a traffic stream and does not worsen, and hopefully improves, traffic congestion for following vehicles. Taking that condition as a given, and with the privacy of the driver in mind, there are a few ways in which the application could be extended.

As a first example, assume that a driver who uses the intelligent feedback application is also always using the same smart phone. By creating a profile of the driver's driving style over time, feedback may be customized to complement that style (Chen et al., 2012). In the context of an embedded system, a small number of static heuristics may be available for the system to use as part of a set of driver 
profiles. The end result would be not just intelligent, but customized feedback for the driver.

A second example involves anonymously sharing information about the traffic stream to a remote service for aggregation with information from other sources, including other smart phones and vehicles using the heuristic. The application could share current time, location, heading, velocity, and recent acceleration curve features. By periodically reporting traffic conditions for an individual vehicle, in particular when an event of note occurs (such as a velocity drop), a synoptic portrait of the roadway may be constructed with greater detail than that available from macroscopic traffic sensors. This overview would be useful in two ways.

First, a forecast of the expected arrival time of an active, traveling shockwave could be communicated to any participating vehicles upstream of the shockwave (Chen et al., 2012). The application could take this knowledge into account and notify the driver of the upcoming congestion, incorporate the knowledge into its own feedback heuristic, or both, amplifying the positive effect over a longer stretch of roadway. An informed driver could, for instance, decide to exit the roadway prior to reaching the congestion and use an alternate route. This could be described as a very specific, lightweight type of connected vehicle technology.

Second, the aggregated view of the traffic stream could be of use to public and private entities involved in traffic management, such as governmental transportation agencies, news agencies, and traveler information services. The combination of data from fixed sensors in an Intelligent Transportation System and floating sensors from smart phones could enhance traffic management and forecasting, improving commuters' experiences. 


\subsection{Final Thoughts}

Autonomous vehicles are several years away from availability and decades away, perhaps, from prevalence. Connected vehicle development lags autonomous vehicle development, though once standards and regulations are adopted, market penetration will be faster. Today, however, a significant percentage of the working public carries a device capable of sensing its environment and improving their commute. Given the magnitude of traffic congestion and the global consequences of inaction, pursuing every avenue toward a more intelligent transportation system is a worthwhile endeavor. 


\section{Appendix A}

\section{A.1 Computing Resources Used}

\begin{tabular}{ccccc}
\hline Host Name & System & CPU Speed & \# CPU Cores & Memory \\
\hline dirk & Dell Poweredge 1950 iii & $3.0 \mathrm{GHz}$ & 8 & $32 \mathrm{~GB}$ \\
lerxst & Dell Poweredge 1950 iii & $3.0 \mathrm{GHz}$ & 8 & $32 \mathrm{~GB}$ \\
pratt & Dell Poweredge 2900 iii & $3.0 \mathrm{GHz}$ & 4 & $48 \mathrm{~GB}$ \\
- & Amazon EC2 c3.8xlarge & $2.8 \mathrm{GHz}$ & 32 & $64 \mathrm{~GB}$ \\
- & Amazon EC2 c3.2xlarge & $2.8 \mathrm{GHz}$ & 8 & $16 \mathrm{~GB}$ \\
- & Amazon EC2 c3.2xlarge & $2.8 \mathrm{GHz}$ & 8 & $16 \mathrm{~GB}$ \\
\hline
\end{tabular}

Table A.1

\section{A.2 Software Used}

Debian "Wheezy" version 7.4 GNU/Linux (Dell Poweredge systems)

Ubuntu "Saucy Salamander" version 13.10 GNU/Linux (Amazon EC2 systems)

Free Software Foundation gcc GNU Compiler Collection version 4.8.2, including the GNU OpenMP Project (GOMP) extension 
libgmp GNU Multiple Precision Arithmetic Library version 5.1.3

libmpfr GNU MPFR Library version 3.1.2

libmpc GNU MPC Library version 1.0.2

libgsl GNU Scientific Library version 1.16

LIBSVM version 3.17

Gnuplot version 4.6 patchlevel 5

TEX version 3.1415926 (TEXLive 2013) 


\section{A.3 Partial Compute Time Records}

\begin{tabular}{|c|c|c|c|c|}
\hline DWT Family & Sample Length & Sample Coefficients & Clock Time & Estimated CPU Hours \\
\hline B-spline 1,3 & 256 & 256 & 0.753889 & 24.1244 \\
\hline B-spline 1,3 & 512 & 512 & 4.27639 & 85.5278 \\
\hline B-spline 1,5 & 256 & 256 & 0.813056 & 26.0178 \\
\hline B-spline 1,5 & 512 & 512 & 4.31889 & 86.3778 \\
\hline B-spline 2,2 & 512 & 512 & 4.07 & 81.4 \\
\hline B-spline 2,4 & 256 & 256 & 0.591389 & 18.9244 \\
\hline B-spline 2,4 & 512 & 512 & 4.16278 & 83.2556 \\
\hline B-spline 2,6 & 256 & 256 & 0.5875 & 18.8 \\
\hline B-spline 2,6 & 512 & 512 & 4.14056 & 82.8111 \\
\hline B-spline 2,8 & 256 & 256 & 0.588333 & 18.8267 \\
\hline B-spline 3,1 & 256 & 256 & 0.576667 & 18.4533 \\
\hline B-spline 3,3 & 256 & 256 & 0.587778 & 18.8089 \\
\hline B-spline 3,3 & 512 & 32 & 19.9194 & 398.389 \\
\hline B-spline 3,3 & 512 & 512 & 0.965 & 30.88 \\
\hline B-spline 3,3 & 512 & 64 & 35.3511 & 707.022 \\
\hline B-spline 3,3 & 512 & 96 & 62.6133 & 1252.27 \\
\hline B-spline 3,5 & 256 & 256 & 0.578333 & 18.5067 \\
\hline B-spline 3,5 & 512 & 512 & 0.949444 & 30.3822 \\
\hline B-spline 3,7 & 256 & 256 & 0.587222 & 18.7911 \\
\hline B-spline 3,7 & 512 & 512 & 0.970556 & 31.0578 \\
\hline B-spline 3,9 & 256 & 256 & 0.580556 & 18.5778 \\
\hline B-spline 3,9 & 512 & 512 & 0.957222 & 30.6311 \\
\hline Daubechies 6 & 256 & 256 & 0.582222 & 18.6311 \\
\hline Daubechies 6 & 512 & 512 & 0.972778 & 31.1289 \\
\hline Daubechies 8 & 256 & 256 & 0.588056 & 18.8178 \\
\hline Daubechies 8 & 512 & 512 & 0.965278 & 30.8889 \\
\hline Daubechies 10 & 256 & 256 & 0.573889 & 18.3644 \\
\hline Daubechies 10 & 512 & 512 & 0.958056 & 30.6578 \\
\hline Daubechies 12 & 256 & 256 & 0.560278 & 17.9289 \\
\hline Daubechies 12 & 512 & 512 & 0.956944 & 30.6222 \\
\hline Daubechies 14 & 256 & 256 & 0.573889 & 18.3644 \\
\hline Daubechies 14 & 512 & 512 & 0.969444 & 31.0222 \\
\hline Daubechies 16 & 256 & 256 & 0.574444 & 18.3822 \\
\hline Daubechies 16 & 512 & 512 & 0.960833 & 30.7467 \\
\hline Daubechies 18 & 256 & 256 & 0.565833 & 18.1067 \\
\hline Daubechies 18 & 512 & 32 & 0.729444 & 14.5889 \\
\hline Daubechies 18 & 512 & 64 & 0.746111 & 14.9222 \\
\hline Daubechies 18 & 512 & 96 & 1.00111 & 20.0222 \\
\hline Daubechies 18 & 512 & 128 & 1.27167 & 25.4333 \\
\hline Daubechies 18 & 512 & 160 & 1.53833 & 30.7667 \\
\hline Daubechies 18 & 512 & 192 & 1.77139 & 35.4278 \\
\hline Daubechies 18 & 512 & 224 & 2.01778 & 40.3556 \\
\hline Daubechies 18 & 512 & 256 & 3.86639 & 77.3278 \\
\hline Daubechies 18 & 512 & 288 & 4.00889 & 80.1778 \\
\hline Daubechies 18 & 512 & 320 & 3.96972 & 79.3944 \\
\hline Daubechies 18 & 512 & 352 & 3.93056 & 78.6111 \\
\hline Daubechies 18 & 512 & 384 & 4.07056 & 81.4111 \\
\hline Daubechies 18 & 512 & 416 & 4.03528 & 80.7056 \\
\hline Daubechies 18 & 512 & 448 & 4.16639 & 83.3278 \\
\hline Daubechies 18 & 512 & 480 & 4.09111 & 81.8222 \\
\hline Daubechies 18 & 512 & 512 & 0.964444 & 30.8622 \\
\hline Daubechies 20 & 256 & 256 & 0.569444 & 18.2222 \\
\hline Daubechies 20 & 512 & 512 & 0.963056 & 30.8178 \\
\hline Haar 2 & 256 & 256 & 0.738611 & 23.6356 \\
\hline Haar 2 & 512 & 512 & 1.0175 & 32.56 \\
\hline Total & & & 204.21 & 4223.04 \\
\hline
\end{tabular}

Table A.2: Elapsed real clock time and estimated CPU hours spent calculating each listed Support Vector Machine. Many more Support Vector Machines were calculated without logging information about their computation. 


\section{Bibliography}

3rd Generation Partnership Project. 3GPP specification: 25.305, Jan. 2013. URL http://www .3gpp.org/ftp/Specs/html-info/25305.htm.

S. Abe. Support Vector Machines for Pattern Classification. Springer, 2010. ISBN 9781849960984. URL http://books . google. com/books?id=Uy1pqRzABDgC.

H. Adeli and X. Jiang. Intelligent infrastructure: neural networks, wavelets, and chaos theory for intelligent transportation systems and smart structures. CRC Press, Boca Raton, 2009. ISBN 97814200853651420085360.

H. Adeli and A. Karim. Wavelets in intelligent transportation systems. Wiley \& Sons, 2005. ISBN 9780470867426. URL http://books.google.com/books?id= lypSAAAAMAAJ.

American Association for the Advancement of Science. What We Know, Mar. 2014. URL http: //whatweknow . aaas .org/.

Y. Amichai-Hamburger. Technology and Psychological Well-being. Cambridge University Press, 2009. ISBN 9781139481052. URL http://books. google.com/books? id=C_If AwAAQBAJ.

Apple, Inc. CMMotionManager Class Reference, Sept. 2013. URL https://developer.apple.com/library/ios/documentation/coremotion/ reference/cmmotionmanager_class/Reference/Reference.html\#//apple_ ref/occ/instp/CMMotionManager/accelerometerUpdateInterval.

R. Arnott and K. Small. The Economics of Traffic Congestion. American Scientist, 82(5):pp. 446-455, 1994. ISSN 00030996. URL http://www. jstor.org/stable/ 29775281.

Automatic. Automatic: Your Smart Driving Assistant, 2013. URL https://www . automatic. com/. 
J. N. Barkenbus. Eco-driving: An overlooked climate change initiative. Energy Policy, 38(2):762 - 769, 2010. ISSN 0301-4215. doi: 10.1016/j.enpol.2009.10.021.

M. Barth and K. Boriboonsomsin. Real-world carbon dioxide impacts of traffic congestion. Transportation Research Record, page 9, 2008.

M. Barth and K. Boriboonsomsin. Traffic Congestion and Greenhouse Gases. Access, 35:2-9, 2009. URL http://www. uctc.net/access/35/access35_Traffic_ Congestion_and_Grenhouse_Gases.shtml.

T. Bauer, J. Dale, F. Offermann, and R. Schwarzmann. Traffic control on german highways - experiences of the efficiency of different control measures on traffic flow. In Institute of Transportation Engineers Annual Meeting and Exhibit 2009, volume 2, pages 842-852, San Antonio, TX, 2009. ISBN 9781615676309.

F. Ben Abdesslem, A. Phillips, and T. Henderson. Less is more: energy-efficient mobile sensing with senseless. In Proceedings of the 1st ACM workshop on Networking, systems, and applications for mobile handhelds, MobiHeld '09, page 61-62, New York, NY, USA, 2009. ACM. ISBN 978-1-60558-444-7. doi: 10.1145/1592606.1592621. URL http://doi .acm.org/10.1145/1592606.1592621.

A. Ben-Hur and J. Weston. A user's guide to support vector machines. Methods in molecular biology (Clifton, N.J.), 609:223-239, 2010. ISSN 19406029. doi: 10.1007/ 978-1-60327-241-4_13.

R. Bhoraskar, N. Vankadhara, B. Raman, and P. Kulkarni. Wolverine: Traffic and road condition estimation using smartphone sensors. 2012 4th International Conference on Communication Systems and Networks, COMSNETS 2012, 2012. ISBN 9781467302982 (ISBN).

E. Brockfeld, R. Kühne, A. Skabardonis, and P. Wagner. Toward Benchmarking of Microscopic Traffic Flow Models. Transportation Research Record, (1852):124-129, 2003. ISSN 03611981.

E. Brockfeld, R. Kühne, and P. Wagner. Calibration and validation of microscopic traffic flow models. Transportation Research Record, (1876):62-70, 2004. ISSN 03611981.

E. Brockfeld, R. Kühne, and P. Wagner. Calibration and validation of microscopic models of traffic flow. Transportation Research Record, (1934):179-187, 2005. ISSN 03611981. 
M. J. Cassidy and R. L. Bertini. Some traffic features at freeway bottlenecks. Transportation Research Part B: Methodological, 33(1):25 - 42, 1999. ISSN 0191-2615. doi: http://dx.doi.org/10.1016/S0191-2615(98)00023-X.

C.-C. Chang and C.-J. Lin. LIBSVM: A library for support vector machines. ACM Transactions on Intelligent Systems and Technology, 2(3):27:1-27:27, 2011.

D. Chen, J. Laval, Z. Zheng, and S. Ahn. A behavioral car-following model that captures traffic oscillations. Transportation Research Part B: Methodological, 46(6): 744-761, 2012. ISSN 01912615 (ISSN).

K. Chen, P. C. o. I. Transport, and J. Miles. ITS handbook 2000: recommendations from the World Road Association (PIARC). Artech House ITS library. Artech House, 1999. ISBN 9781580531030. URL http://books . google.com/books?id=tShPAAAAMAAJ.

H. Chesbrough. Open Services Innovation: Rethinking Your Business to Grow and Compete in a New Era. John Wiley \& Sons, Dec. 2010. ISBN 9780470949337.

D. Chowdhury, L. Santen, and A. Schadschneider. Statistical physics of vehicular traffic and some related systems. Physics Report, 329(4-6):199-329, 2000. ISSN 03701573.

Cisco. Cisco Visual Networking Index: Global Mobile Data Traffic Forecast Update, 2013-2018, Feb. 2014. URL http://cisco.com/c/en/us/solutions/ collateral/service-provider/visual-networking-index-vni/white_paper_ c11-520862.html.

M. Coelho, T. Farias, and N. Rouphail. A numerical tool for estimating pollutant emissions and vehicles performance in traffic interruptions on urban corridors. International Journal of Sustainable Transportation, 3(4):246-262, 2009. ISSN 15568318 (ISSN).

M. C. Coelho. Assessment and Modeling of Traffic Performance and Emission Impacts of Traffic Interruptions in Urban Corridors. PhD thesis, Doctoral Dissertation, Instituto Superior Técnico-Technical University of Lisbon, Portugal, 2005.

R. Colombo and A. Groli. Minimising stop and go waves to optimise traffic flow. Applied Mathematics Letters, 17(6):697-701, 2004. ISSN 08939659 (ISSN).

J. Cook, D. Nuccitelli, S. Green, M. Richardson, B. Winkler, R. Painting, R. Way, P. Jacobs, and A. Skuce. Quantifying the consensus on anthropogenic global warming in the scientific literature. Environmental Research Letters, 8(2), 2013. ISSN 17489318. doi: 10.1088/1748-9326/8/2/024024. 
Corona Labs Inc. Corona Docs, Jan. 2014. URL http: //docs . coronalabs . com/api/ library/system/setAccelerometerInterval.html.

J. Dai, J. Teng, X. Bai, Z. Shen, and D. Xuan. Mobile phone based drunk driving detection. In 2010 4th International Conference on Pervasive Computing Technologies for Healthcare, Pervasive Health 2010, Munich, 2010. ISBN 9789639799899. doi: 10.4108/ICST.PERVASIVEHEALTH2010.8901.

M. DeGusta. Are Smart Phones Spreading Faster than Any Technology in Human History?, May 2012. URL http://www.technologyreview.com/news/427787/ are-smart-phones-spreading-faster-than-any-technology-in-human-history/.

A. Downs. Still stuck in traffic: coping with peak-hour traffic congestion. James A. Johnson metro series. Brookings Institution Press, Washington, D.C, 2004. ISBN 0815719299 .

DriveGain Limited. DriveGain, 2011. URL http://drivegain.com/.

G. Duncan and J. Littlejohn. High performance microscopic simulation for traffic forecasting. In Anon, editor, IEE Colloquium (Digest), pages 4/1-4/2, London, UK, 1997. IEE, Stevenage, United Kingdom.

S. Elkatsha. Cairo Drive, Oct. 2013. URL https://twitter.com/CairoDriveFilm.

L. Evans. Driver Behavior Effects on Fuel Consumption in Urban Driving. Human Factors, 21(4):389-398, 1979. ISSN 00187208.

M. Fazeen, B. Gozick, R. Dantu, M. Bhukhiya, and M. González. Safe Driving Using Mobile Phones. IEEE Transactions on Intelligent Transportation Systems, 2012. ISSN 15249050. doi: 10.1109/TITS.2012.2187640.

Findra Studio. Eco Fuel Calculator, Mar. 2014. URL https://play.google.com/ store/apps/details?id=com.findra.fuelcal.

Free Software Foundation, Inc. GNU Scientific Library, July 2013. URL http: //www.gnu.org/software/gsl/.

Free Software Foundation, Inc. GNU Compiler Collection, Apr. 2014. URL http: //gcc.gnu.org/.

H. C. Frey, A. Unal, N. M. Rouphail, and J. D. Colyar. On-Road Measurement of Vehicle Tailpipe Emissions Using a Portable Instrument. Journal of the Air \& Waste Management Association, 53(8):992-1002, 2003. doi: 10.1080/ 10473289.2003.10466245. URL http://www.tandfonline.com/doi/abs/10.1080/ 10473289.2003 .10466245 . 
D. C. Gazis, R. Herman, and R. W. Rothery. Nonlinear Follow-the-Leader Models of Traffic Flow. Operations Research, 9(4):545-567, July 1961. ISSN 0030-364X, 1526-5463. doi: 10.1287/opre.9.4.545. URL http://or . journal.informs.org/ cgi/doi/10.1287/opre.9.4.545.

I. M. Gelfand and S. V. Fomin. Calculus of variations. Courier Dover Publications, 2000.

General Motors. 'Super Cruise' Takes on Real-World Traffic Scenarios, Apr. 2013. URL http://media.gm.com/media/us/en/gm/news.detail.html/ content/Pages/news/us/en/2013/Apr/0429-cadillac-super-cruise .html.

R. Gibbens and F. Kelly. An investigation of proportionally fair ramp metering. In IEEE Conference on Intelligent Transportation Systems, Proceedings, ITSC, 14th IEEE International Intelligent Transportation Systems Conference, ITSC 2011, pages 490-495, Washington, DC, 2011. ISBN 9781457721984. doi: 10.1109/ITSC.2011. 6082812 .

R. Gibbens and Y. Saatci. Data, modelling and inference in road traffic networks. Philosophical Transactions of the Royal Society A: Mathematical, Physical and Engineering Sciences, 366(1872):1907-1919, 2008. ISSN 1364503X. doi: 10.1098/rsta.2008.0020.

W. Gibson. Talk of the Nation, Nov. 1999. URL http://www.npr.org/templates/ story/story .php?storyId=1067220.

E. L. Glaeser, M. E. Kahn, and J. Rappaport. Why do the poor live in cities? The role of public transportation. Journal of Urban Economics, 63(1):1 - 24, 2008. ISSN 0094-1190. doi: http://dx.doi.org/10.1016/j.jue.2006.12.004.

Global Industry Analysts, Inc. Adaptive Cruise Control Systems - A Global Strategic Business Report. Technical Report MCP-1181, Global Industry Analysts, Inc., Feb. 2012. URL http://www . strategyr .com/Adaptive_Cruise_Control_ Systems_Market_Report.asp.

J. Gonder, M. Earleywine, and W. Sparks. Analyzing Vehicle Fuel Saving Opportunities through Intelligent Driver Feedback. SAE International Journal of Passenger Cars - Electronic and Electrical Systems, 5(2), 2012. ISSN 19464614 (ISSN). URL http://papers.sae.org/2012-01-0494/.

J. Graving, M. Rakauskas, M. Manser, and J. Jenness. A binary response method to determine the usability of seven in-vehicle fuel economy displays. In Proceedings of the Human Factors and Ergonomics Society, volume 2, pages 1546-1550, San Francisco, CA, 2010. ISBN 9781617820885. doi: 10.1518/107118110X12829370088921. 
F. L. Hall and K. Agyemang-Duah. Freeway capacity drop and the definition of capacity. Transportation Research Record, (1320), 1991.

D. Helbing. Traffic and related self-driven many-particle systems. Rev. Mod. Phys., 73(4):1067-1141, Dec. 2001. doi: 10.1103/RevModPhys.73.1067. URL http:// link.aps.org/doi/10.1103/RevModPhys.73.1067.

D. Hennessy and D. Wiesenthal. Traffic congestion, driver stress, and driver aggression. Aggressive Behavior, 25(6):409-423, 1999. ISSN 0096140X. doi: 10.1002/(SICI) 1098-2337(1999)25:6 \ensuremath\{<\}409::AID-AB2 \ensuremath\{>\}3.0.CO;2-0.

S. Hoogendoorn and R. Hoogendoorn. Calibration of microscopic traffic-flow models using multiple data sources. Philosophical Transactions of the Royal Society A: Mathematical, Physical and Engineering Sciences, 368(1928):4497-4517, 2010. ISSN 1364503X. doi: 10.1098/rsta.2010.0189.

C.-W. Hsu, C.-C. Chang, and C.-J. Lin. A Practical Guide to Support Vector Classification, Apr. 2010. URL http://www. csie.ntu.edu.tw/ ${ }^{c j l i n / p a p e r s / g u i d e / ~}$ guide.pdf.

Hunter Research \& Technology. greenMeter: iPhone/iPod Eco-driving App, 2014. URL http://hunter . pairsite.com/greenmeter/.

Y. Igarashi, K. Itoh, K. Nakanishi, K. Ogura, and K. Yokokawa. Bifurcation phenomena in the optimal velocity model for traffic flow. Physical Review E - Statistical, Nonlinear, and Soft Matter Physics, 64(4 II):471021-471024, 2001. ISSN 15393755.

IndustryARC. Global Advanced Driver Assistance Systems (ADAS) Market Analysis and Forecast (2013 -2018): Reduced Costs and Customer Demand Shows Immense Growth Potential for Market, Sept. 2013. URL http://industryarc . com/Report/20/advanced-driver-assistance-systems-market.html.

INRIX. New INRIX Gridlock Index (IGI) Shows Traffic Congestion Rising with the Economy, Dec. 2012. URL http: //www . inrix. com/pressrelease . asp? ID=182.

International Transport Forum and International Energy Agency. ITF/IEA Workshop on Ecodriving. Technical report, Organisation for Economic Co-operation and Development, Paris, France, Nov. 2007. URL http://www . internationaltransportforum.org/Proceedings/ecodriving/ ecodriving07.html.

Ishida R\&D. OBD Mileage, Oct. 2013. URL http://www.tabinomado.jp/n_en/. 
M. Islam, M. Hadiuzzaman, L. Ying, T. Qiu, and K. El-Basyouny. An investigation of the relationship between speed characteristics and collision rate for urban freeway. In CICTP 2012: Multimodal Transportation Systems - Convenient, Safe, Cost-Effective, Efficient - Proceedings of the 12th COTA International Conference of Transportation Professionals, pages 3498-3506, Beijing, 2012. ISBN 9780784412442. doi: 10.1061/9780784412442.354.

A. Jensen and A. Cour-Harbo. Ripples in Mathematics: The Discrete Wavelet Transform. Springer, 2001. ISBN 9783540416623. URL http://books.google.com/books? id=hMvhjWxb0_MC.

K. Jerath and S. Brennan. Analytical prediction of self-organized traffic jams as a function of increasing ACC penetration. IEEE Transactions on Intelligent Transportation Systems, 13(4):1782-1791, 2012. ISSN 15249050. doi: 10.1109/TITS.2012. 2217742.

D. Johnson and M. Trivedi. Driving style recognition using a smartphone as a sensor platform. 14th IEEE International Intelligent Transportation Systems Conference, ITSC 2011, pages 1609-1615, 2011. ISBN 9781457721984 (ISBN).

M. Jones and M. Appleby. Driver Performance and Fuel Economy. In Proceedings of the Human Factors and Ergonomics Society Annual Meeting, volume 22, page 539-540, 1978.

N. Kalra, J. Anderson, and M. Wachs. Liability and regulation of autonomous vehicle technologies. California PATH Program, Institute of Transportation Studies, University of California at Berkeley, 2009.

A. Kesting and M. Treiber. How reaction time, update time, and adaptation time influence the stability of traffic flow. Computer-Aided Civil and Infrastructure Engineering, 23(2):125-137, 2008. ISSN 10939687. doi: 10.1111/j.1467-8667.2007. 00529.x.

A. Kesting, M. Treiber, and D. Helbing. General lane-changing model MOBIL for car-following models. Transportation Research Record: Journal of the Transportation Research Board, 1999(1):86-94, 2007a.

A. Kesting, M. Treiber, M. Schönhof, F. Kranke, and D. Helbing. Jam-Avoiding Adaptive Cruise Control (ACC) and its Impact on Traffic Dynamics. In A. Schadschneider, T. Pöschel, R. Kühne, M. Schreckenberg, and D. Wolf, editors, Traffic and Granular Flow'05, pages 633-643. Springer Berlin Heidelberg, 2007b. ISBN 978-3-540-47640-5. URL http://dx.doi.org/10.1007/978-3-540-47641-2_62. 
A. Kesting, M. Treiber, and D. Helbing. Agents for Traffic Simulation. ArXiv e-prints, May 2008a.

A. Kesting, M. Treiber, M. Schönhof, and D. Helbing. Adaptive cruise control design for active congestion avoidance. Transportation Research Part C: Emerging Technologies, 16(6):668 - 683, 2008b. ISSN 0968-090X. doi: 10.1016/j.trc.2007.12.004.

A. Kesting, M. Treiber, and D. Helbing. Enhanced intelligent driver model to access the impact of driving strategies on traffic capacity. Philosophical Transactions of the Royal Society A: Mathematical, Physical and Engineering Sciences, 368(1928): 4585-4605, 2010. ISSN 1364503X. doi: 10.1098/rsta.2010.0084.

W. Knight. A Test Drive of the Most Advanced Driverless Cars, Oct. 2013. URL http://www.technologyreview.com/featuredstory/520431/ driverless-cars-are-further-away-than-you-think/.

W. Knospe, L. Santen, A. Schadschneider, and M. Schreckenberg. Empirical test for cellular automaton models of traffic flow. Physical Review E - Statistical, Nonlinear, and Soft Matter Physics, 70(1 2):016115-1-016115-25, 2004. ISSN 15393755. doi: 10.1103/PhysRevE.70.016115.

M. Koslowsky, A. Kluger, and M. Reich. Commuting Stress: Causes, Effects and Methods of Coping. Springer Series on Stress and Coping. Springer, 1995. ISBN 9780306450372. URL http://books . google . com/books?id=1qIP4Ya4F2IC.

N. Lane, E. Miluzzo, H. Lu, D. Peebles, T. Choudhury, and A. Campbell. A survey of mobile phone sensing. Communications Magazine, IEEE, 48(9):140-150, Sept. 2010. ISSN 0163-6804. doi: 10.1109/MCOM.2010.5560598.

T. Lassa. 2014 Technology of the Year: Autonomy, Nov. 2013. URL http://www . automobilemag.com/features/awards/1401_2014_technology_ of_the_year_autonomy/.

J. Laval and L. Leclercq. A mechanism to describe the formation and propagation of stop-and-go waves in congested freeway traffic. Philosophical Transactions of the Royal Society A: Mathematical, Physical and Engineering Sciences, 368(1928): 4519-4541, 2010. ISSN 1364503X (ISSN).

G. Leen and D. Heffernan. Expanding automotive electronic systems. Computer, 35 (1):88-93, 2002. ISSN 00189162. doi: 10.1109/2.976923. 
D. Levinson and L. Zhang. Ramp meters on trial: Evidence from the Twin Cities metering holiday. Transportation Research Part A: Policy and Practice, 40(10):810828, Dec. 2006. ISSN 09658564. doi: 10.1016/j.tra.2004.12.004. URL http:// linkinghub.elsevier.com/retrieve/pii/S0965856406000024.

D. Levinson, K. Harder, J. Bloomfield, and K. Winiarczyk. Weighting waiting: Evaluating perception of in-vehicle travel time under moving and stopped conditions. Transportation Research Record, (1898):61-68, 2004. ISSN 03611981.

D. Lewis. Estimating the influence of public policy on road traffic levels in Greater London. Journal of Transport Economics and Policy, 11(2):155-168, 1977. ISSN 00225258. URL http://www.jstor.org/stable/20052468.

M. J. Lighthill and G. B. Whitham. On kinematic waves. II. A theory of traffic flow on long crowded roads. Proceedings of the Royal Society of London. Series A. Mathematical and Physical Sciences, 229(1178):317-345, 1955.

T. Litman. Congestion Costing Critique. Victoria Transport Policy Institute, 2013.

H. Lu, J. Yang, Z. Liu, N. Lane, T. Choudhury, and A. Campbell. The Jigsaw continuous sensing engine for mobile phone applications. In SenSys 2010 - Proceedings of the 8th ACM Conference on Embedded Networked Sensor Systems, pages 71-84, Zurich, 2010. ISBN 9781450303446. doi: 10.1145/1869983.1869992.

J. Ma, F. Zhou, and M. Demetsky. Evaluating mobility and sustainability benefits of cooperative adaptive cruise control using agent-based modeling approach. 2012 IEEE Systems and Information Engineering Design Symposium, SIEDS 2012, pages 74-78, 2012. ISBN 9781467312851 (ISBN).

J. Maddox. Improving Driving Safety Through Automation, presentation at the Congressional Robotics Caucus. National Highway Traffic Safety Administration, 2012.

S. Maerivoet. Traffic Cellular Automata+, 2005. URL http://smtca.dyns .cx/.

S. Maerivoet and B. De Moor. Transportation Planning and Traffic Flow Models. ArXiv Physics e-prints, July 2005.

R. McGrath. The Pace of Technology Adoption is Speeding Up, Nov. 2013. URL http://blogs.hbr.org/2013/11/ the-pace-of-technology-adoption-is-speeding-up/.

A. McKinnon and J. Edwards. Opportunities for improving vehicle utilization. Green Logistics: Improving the Environmental Sustainability of Logistics, page 195-213, 2010. 
J. M. Melillo, T. T. Richmond, and G. W. Yohe. Climate Change Impacts in the United States: The Third National Climate Assessment. Technical report, U.S. Global Change Research Program, Washington, D.C, May 2014. URL http: //nca2014.globalchange.gov/.

Mercedes-Benz USA. M-Class - Interactive Owner's Manual, 2013. URL http://www4.mercedes-benz.com/manual-cars/ba/cars/w166/en/overview/ fahrsysteme8.html.

G. Meyer and S. Beiker. Road Vehicle Automation. Lecture Notes in Mobility. Springer, Aug. 2014. ISBN 978-3-319-05989-1. URL http://www . springer. com/ engineering/mechanical+engineering/book/978-3-319-05989-1.

T. Mitchell. Machine Learning. McGraw-Hill international editions - computer science series. McGraw-Hill Education, 1997. ISBN 9780070428072. URL http: //books . google. com/books?id=xOGAngEACAAJ.

M. J. Mogridge. Travel in Towns. Jam Yesterday, Jam Today and Jam Tomorrow? MacMillan, 1990. ISBN 0-333-53204-X. URL http://trid.trb.org/view.aspx?id= 350619.

P. Mohan, V. N. Padmanabhan, and R. Ramjee. Nericell: rich monitoring of road and traffic conditions using mobile smartphones. In Proceedings of the 6th ACM conference on Embedded network sensor systems, SenSys '08, page 323-336, New York, NY, USA, 2008. ACM. ISBN 978-1-59593-990-6. doi: 10.1145/1460412.1460444. URL http://doi . acm.org/10.1145/1460412.1460444.

NationalAtlas.gov. Transportation of the United States, Jan. 2013. URL http: //nationalatlas.gov/transportation.html.

Nevada State Legislature. Autonomous Vehicles, July 2013. URL http://www .leg. state.nv.us/NRS/NRS-482A.html.

G. Nirschl. Human-Centered Development of Advanced Driver Assistance Systems. In M. J. Smith and G. Salvendy, editors, Human Interface and the Management of Information. Interacting in Information Environments, volume 4558 of Lecture Notes in Computer Science, pages 1088-1097. Springer Berlin Heidelberg, 2007. ISBN 978-3-540-73353-9. URL http://dx. doi .org/10.1007/978-3-540-73354-6_119.

R. W. Novaco and O. I. Gonzalez. Commuting and Well-Being. In Technology and Psychological Well-Being. Cambridge University Press, 2009. 
M. P. O'Keefe, A. Simpson, K. J. Kelly, and D. S. Pedersen. Duty Cycle Characterization and Evaluation Towards Heavy Hybrid Vehicle Applications. In SP-2101, Detroit, Michigan, Apr. 2007. doi: 10.4271/2007-01-0302. URL http://papers . sae.org/2007-01-0302/.

G. Orosz, R. Wilson, R. Szalai, and G. Stépán. Exciting traffic jams: Nonlinear phenomena behind traffic jam formation on highways. Physical Review E Statistical, Nonlinear, and Soft Matter Physics, 80(4), 2009. ISSN 15393755. doi: 10.1103/PhysRevE.80.046205.

G. Orosz, R. E. Wilson, and G. Stepan. Traffic jams: Dynamics and control. Philosophical Transactions of the Royal Society A: Mathematical, Physical and Engineering Sciences, 368(1928):4455-4479, 2010. ISSN 1364503X (ISSN).

K. Öğüt and J. Banks. Stability of freeway bottleneck flow phenomena. Transportation Research Record, (1934):108-115, 2005. ISSN 03611981.

R. K. Pachauri and Intergovernmental Panel on Climate Change. Climate change 2007: synthesis report: a report of the Intergovernmental Panel on Climate Change. IPCC, WMO, Geneva, 2008. ISBN 92916912249789291691227.

R. Parasuraman and V. Riley. Humans and automation: Use, misuse, disuse, abuse. Human Factors, 39(2):230-253, 1997. ISSN 00187208. doi: 10.1518/ 001872097778543886.

B. Rebsamen, T. Bandyopadhyay, T. Wongpiromsarn, S. Kim, Z. Chong, B. Qin, M. Ang, E. Frazzoli, and D. Rus. Utilizing the infrastructure to assist autonomous vehicles in a mobility on demand context. 2012.

P. Richards. Shockwaves on the highway. Operations Research, 4:42-51, 1956.

A. Riener and A. Ferscha. Effect of proactive braking on traffic flow and road throughput. 13th IEEE/ACM Symposium on Distributed Simulation and RealTime Applications, DS-RT 2009, pages 157-164, 2009. ISBN 15506525 (ISSN); 9780769538686 (ISBN).

R. Riley. Alternative cars in the 21st century: A new personal transportation. Society of Automotive Engineers, 1 edition, Dec. 1994. ISBN 9781560915195. URL http://www.osti.gov/scitech/servlets/purl/418700.

T. W. Sanchez. Poverty, policy, and public transportation. Transportation Research Part A: Policy and Practice, 42(5):833 - 841, 2008. ISSN 0965-8564. doi: http: //dx.doi.org/10.1016/j.tra.2008.01.011. 
ScanGauge. ScanGauge - Trip Computer + Digitial Gauges + ScanTools, 2013. URL http://www.scangauge.com/.

A. Schadschneider, D. Chowdhury, and K. Nishinari. Stochastic transport in complex systems from molecules to vehicles. Elsevier, Amsterdam; Oxford, 2011. ISBN 9780444528537044452853997800805605260080560520 . URL http://public. eblib.com/EBLPublic/PublicView.do?ptiID=631944.

B. Schaller. New York City's congestion pricing experience and implications for road pricing acceptance in the United States. Transport Policy, 17(4):266-273, Aug. 2010. ISSN 0967070X. doi: 10.1016/j.tranpol.2010.01.013. URL http://linkinghub. elsevier.com/retrieve/pii/S0967070X10000326.

D. Schrank, B. Eisele, and T. Lomax. Annual Urban Mobility Report. Technical report, Texas Transportation Institute, 2012. URL http://mobility. tamu .edu/ ums/.

C. Seligman. Feedback as an energy conservation strategy. Proceedings of the Human Factors Society 22nd Annual Meeting, page 537, 1978.

O. Servin, K. Boriboonsomsin, and M. Barth. An energy and emissions impact evaluation of intelligent speed adaptation. In IEEE Conference on Intelligent Transportation Systems, Proceedings, ITSC, pages 1257-1262, Toronto, ON, 2006. ISBN $1424400945 ; 9781424400942$.

J. Shibata and R. French. A Comparison of Intelligent Transportation Systems: Progress Around the World Through 1996. Publication ITS-AMER-97-B011. ITS America, 1997. URL http: //books . google.com/books?id=1ja9HAAACAAJ.

S. E. Shladover. Longitudinal control of automotive vehicles in close-formation platoons. Journal of dynamic systems, measurement, and control, 113(2):231-241, 1991.

D. C. Shoup, A. P. Association, and others. The high cost of free parking, volume 7. Planners Press, American Planning Association Washington, DC, USA:, 2005.

M. Sifuzzaman, M. R. Islam, and M. Z. Ali. Application of wavelet transform and its advantages compared to Fourier transform. 2009.

G. Silberg and R. Wallace. Self-Driving Cars -The Next Revolution, 2012. URL http://www.kpmg.com/us/en/issuesandinsights/articlespublications/ pages/self-driving-cars-next-revolution.aspx.

A. Smith. $46 \%$ of American adults are smartphone owners. Pew Internet $\mathcal{E}$ American Life Project, 2012. 
M. J. Smith and G. Salvendy. Human Interface and the Management of Information. Interacting in Information Environments, volume 4558 of Lecture Notes in Computer Science. Springer Berlin Heidelberg, Beijing, China, 2007. ISBN 987-3-540-73353-9. URL http: //link. springer. com/book/10.1007\%2F978-3-540-73354-6.

J. Sousanis. World Vehicle Population Tops 1 Billion Units I News \& Analysis content from WardsAuto, Aug. 2011. URL http://wardsauto.com/ar/world_ vehicle_population_110815.

K. Spieser, K. B. Treleaven, R. Zhang, E. Frazzoli, D. Morton, and M. Pavone. Toward a Systematic Approach to the Design and Evaluation of Automated Mobilityon-Demand Systems: A Case Study in Singapore. In Road Vehicle Automation, Lecture Notes in Mobility. Springer, Aug. 2014. ISBN 978-3-319-05989-1. URL http://hdl . handle. net/1721.1/82904.

I. Steinwart and A. Christmann. Support Vector Machines. Springer, 2008. ISBN 9780387772424. URL http://books . google . com/books?id=HUnqnrpYt4IC.

STMicroelectronics. LIS331DLH MEMS motion sensor: Ultra low power high performance 3-axis digital accelerometer - STMicroelectronics, 2014a. URL http: //www.st.com/web/catalog/sense_power/FM89/SC444/PF218132.

STMicroelectronics. L3G4200D MEMS motion sensor: three-axis digital output gyroscope - STMicroelectronics, 2014b. URL http://www. st .com/web/catalog/ sense_power/FM89/SC1288/PF250373.

T. Stocker, Intergovernmental Panel on Climate Change, and Working Group I. Climate change 2013: the physical science basis : Working Group I contribution to the fifth assessment report of the Intergovernmental Panel on Climate Change. 2013. ISBN $9781107661820110766182 X$.

Y. Sugiyamal, M. Fukui, M. Kikuchi, K. Hasebe, A. Nakayama, K. Nishinari, S.-I. Tadaki, and S. Yukawa. Traffic jams without bottlenecks-experimental evidence for the physical mechanism of the formation of a jam. New Journal of Physics, 10, 2008. ISSN 13672630. doi: 10.1088/1367-2630/10/3/033001.

Transportation Research Board. TRB HCM Manuals Home, 2011. URL http: //hcm.trb.org/.

Transportation Research Board. Transport Research International Documentation, 2014. URL http://trid.trb.org/.

M. Treiber. Dynamic traffic simulation, June 2011. URL http://www . traffic-simulation.de/. 
M. Treiber and D. Helbing. Explanation of Observed Features of Self-Organization in Traffic Flow. eprint arXiv:cond-mat/9901239, Jan. 1999.

M. Treiber and A. Kesting. An open-source microscopic traffic simulator. IEEE Intelligent Transportation Systems Magazine, 2(3):6-13, 2010. doi: 10.1109/MITS. 2010.939208.

M. Treiber, A. Hennecke, and D. Helbing. Congested traffic states in empirical observations and microscopic simulations. Physical Review E - Statistical Physics, Plasmas, Fluids, and Related Interdisciplinary Topics, 62(2 B):1805-1824, 2000. ISSN 1063651X.

M. Treiber, A. Kesting, and D. Helbing. Delays, inaccuracies and anticipation in microscopic traffic models. Physica A: Statistical Mechanics and its Applications, 360 (1):71-88, 2006a. ISSN 03784371. doi: 10.1016/j.physa.2005.05.001.

M. Treiber, A. Kesting, and D. Helbing. Understanding widely scattered traffic flows, the capacity drop, and platoons as effects of variance-driven time gaps. Physical Review E - Statistical, Nonlinear, and Soft Matter Physics, 74(1), 2006b. ISSN 15393755. doi: 10.1103/PhysRevE.74.016123.

C. Tucker, R. Tucker, and J. Zheng. StopWatcher: A mobile application to improve stop sign awareness for driving safety. International Journal of Vehicular Technology, 2012, 2012. ISSN 16875702. doi: 10.1155/2012/532568.

D. I. S. U. S. Census Bureau. Commuting (Journey to Work) Main, Sept. 2011. URL http://www. census. gov/hhes/commuting/.

A. M. Uhrmacher and D. Weyns. Multi-Agent Systems: Simulation and Applications. CRC Press, Inc., Boca Raton, FL, USA, 1st edition, June 2009. ISBN 1420070231, 9781420070231. URL http://www. crcpress. com/product/isbn/9781420070231.

UltraGauge. UltraGauge OBDII Scan tool \& Information Center, 2014. URL http: //www.ultra-gauge.com.

Universitat Politècnica de València and Grupo de Redes de Computadores. Driving Styles, 2014. URL http://www .drivingstyles .info/index.php/en/.

U.S. DoT RITA ITS Joint Program Office. Safety Pilot, Mar. 2014a. URL http: //www.its.dot.gov/safety_pilot/spmd.htm.

U.S. DoT RITA ITS Joint Program Office. Dedicated Short Range Communications, Mar. 2014b. URL http://www.its.dot.gov/DSRC/index.htm. 
R. Vadde, D. Sun, J. Sai, M. Faruqi, and P. Leelani. A simulation study of using active traffic management strategies on congested freeways. Journal of Modern Transportation, 20(3):178-184, 2012. ISSN 2095087X. doi: 10.3969/j.issn.2095-087X. 2012.03.008.

M. van der Voort. Generating a new fuel efficiency support tool - Motivation, state-of-the-art and salient feautures. CTS Working Paper 1997:9, 1997.

M. van der Voort, M. Dougherty, and M. Van Maarseveen. A prototype fuelefficiency support tool. Transportation Research Part C: Emerging Technologies, 9(4): 279-296, 2001. ISSN 0968090X. doi: 10.1016/S0968-090X(00)00038-3.

T. Vanderbilt. Traffic. Knopf Doubleday Publishing Group, 2008. ISBN 9780307270542. URL http://books . google. com/books?id=041sPQttQ5IC.

P. Varaiya. What we've learned about highway congestion. Access, 27:2-9, 2005.

M. Vollrath, S. Schleicher, and C. Gelau. The influence of Cruise Control and Adaptive Cruise Control on driving behaviour - A driving simulator study. Accident Analysis and Prevention, 43(3):1134-1139, 2011. ISSN 00014575 (ISSN).

C. Wang, M. Quddus, and S. Ison. A spatio-temporal analysis of the impact of congestion on traffic safety on major roads in the UK. Transportmetrica A: Transport Science, 9(2):124-148, 2013. ISSN 23249935. doi: 10.1080/18128602.2010.538871.

J. White, C. Thompson, H. Turner, B. Dougherty, and D. Schmidt. WreckWatch: Automatic traffic accident detection and notification with smartphones. Mobile Networks and Applications, 16(3):285-303, 2011. ISSN 1383469X (ISSN).

R. Wilson. Mechanisms for spatio-temporal pattern formation in highway traffic models. Philosophical Transactions of the Royal Society A: Mathematical, Physical and Engineering Sciences, 366(1872):2017-2032, 2008. ISSN 1364503X (ISSN).

H. Yeo and A. Skabardonis. Understanding Stop-and-go Traffic in View of Asymmetric Traffic Theory. In W. H. K. Lam, S. C. Wong, and H. K. Lo, editors, Transportation and Traffic Theory 2009: Golden Jubilee, pages 99-115. Springer US, 2009. ISBN 978-1-4419-0819-3. URL http://dx.doi.org/10.1007/978-1-4419-0820-9_6.

J. Yeon, S. Hernandez, and L. Elefteriadou. Differences in freeway capacity by day of the week, time of day, and segment type. Journal of Transportation Engineering, 135(7):416-426, 2009. ISSN 0733947X. doi: 10.1061/(ASCE)0733-947X(2009)135: 7(416).

D. Yergin. The prize: The epic quest for oil, money \& power. Simon and Schuster, 2011. 
K. Young and M. Regan. Driver Distraction: A review of the literature. Distracted Driving, pages 379-405, 2007.

M. S. Young and N. A. Stanton. Malleable attentional resources theory: A new explanation for the effects of mental underload on performance. Human Factors, 44(3):365-375, 2002. ISSN 00187208.

H. Zhang. A mathematical theory of traffic hysteresis. Transportation Research Part B: Methodological, 33B(1):1-23, 1999. ISSN 01912615.

X. Zhang, D. Zhao, P. Han, and J. Shen. Multiple linear regression-based approach to the analysis of multi-anticipative car-following behavior. 3rd International Conference on Transportation Engineering, ICTE 2011, pages 156-161, 2011. ISBN 9780784411841 (ISBN).

Z. Zheng, S. Ahn, and C. Monsere. Impact of traffic oscillations on freeway crash occurrences. Accident Analysis and Prevention, 42(2):626-636, 2010. ISSN 00014575 (ISSN).

Z. Zheng, S. Ahn, D. Chen, and J. Laval. Applications of wavelet transform for analysis of freeway traffic: Bottlenecks, transient traffic, and traffic oscillations. Transportation Research Part B: Methodological, 45(2):372-384, 2011. ISSN 01912615 (ISSN).

J. Zhou and H. Peng. Range policy of adaptive cruise control vehicles for improved flow stability and string stability. IEEE Transactions on Intelligent Transportation Systems, 6(2):229-237, 2005. ISSN 15249050. doi: 10.1109/TITS.2005.848359.

B. Zielke, R. Bertini, and M. Treiber. Empirical measurement of freeway oscillation characteristics: An international comparison. Transportation Research Record, (2088):57-67, 2008. ISSN 03611981. doi: 10.3141/2088-07. 